\title{
Mean-field thermodynamic quantum time-space crystal: spontaneous breaking of time-translation symmetry in a macroscopic fermion system.
}

\author{
Konstantin B. Efetov \\ Ruhr University Bochum, Faculty of Physics and Astronomy, Bochum, 44780, Germany \\ National University of Science and Technology "MISiS", Moscow, 119049, Russia and \\ International Institute of Physics, UFRN, 59078-400 Natal, Brazil
}

(Dated: November 5, 2019)

\begin{abstract}
A model demonstrating existence of a thermodynamically stable quantum time-space crystal has been proposed and studied. This state is characterized by an order parameter periodic in both real and imaginary times. The average of the order parameter over phases of the oscillations vanishes but correlation functions of two or more order parameters show non-decaying oscillations. An alternative interpretation of the results is based on a concept of an operator order parameter introduced for this purpose. The model studied here has been suggested previously, in particular, for describing the pseudogap state in superconducting cuprates. Although many properties of the time-space crystal considered here are close to those of a well known DDW state, static magnetic moments oscillating at $(\pi, \pi)$ do not exist. Instead, $\delta$-peaks at finite energies are predicted in the cross-section of inelastic spin-polarized neutron scattering.

PACS numbers: 11.30.-j,05.30.-d,71.10.-w,03.75.-Lm
\end{abstract}

\section{INTRODUCTION}

\section{A. What are thermodynamic quantum time-crystals?}

Crystalline structures play a very important role in modern condensed matter physics and material science. These can be periodic arrays of atoms in metals and insulators but very often they arise as a result of a sharp phase transition. Antiferromagnets, charge density waves and many other states of matter can serve as well known examples of space crystals. If a space crystal appears at a certain critical temperature or a critical parameter characterizing the system one can expect sharp changes of physical quantities at the critical point. The standard way of describing a phase transition is based on the concept of an order parameter introduced by Landau ${ }^{1}$. This quantity equals zero in the disordered phase but is finite in the ordered one.

At the same time, one should be a little bit more careful with this definition because the formal average of the order parameter over thermodynamic states can strictly speaking be equal to zero due to the degeneracy of the free energy functional at the minimum. Indeed, the average magnetic moment of an antiferromagnet or the charge of a charge density wave (CDW) equals zero because the energies of the states of the structures shifted by, e.g., half a period are equal to each other and the average vanishes. We write this property in the form

$$
\int \rho\left(\mathbf{r}-\mathbf{r}_{0}\right) d \mathbf{r}_{0}=0
$$

where, for example, $\rho(\mathbf{r})=\rho_{0} \cos (\mathbf{Q r})$ can be the order parameter of a CDW. The integration is performed over the period along a primitive vector and Eq. 1.1 should be valid for any direction. Of course, depending on the system under consideration there can be additional types of averaging over the degeneracies of the ground state. The energy of a superconductor does not depend on phase and averaging over the phase gives zero, the direction of the antiferromagnetic moment can be arbitrary and the average over the directions equals zero. One can easily continue the list of examples but, to simplify the discussion, we will have in mind the averaging as it is written in Eq. (1.1).

One may ask whether it makes a sense or not to average over the degeneracies of the ground state. Actually, this depends on the type of the experiment designed to probe the material. In many situations, one considers systems with contacts selecting one of the states. However, probing a system with the help of e.g. X-ray scattering one detects a contribution coming from many parts of a sample, and the order parameter can have different phases in different parts of it. Then, one should average over the position of CDW.

In order to avoid the ambiguity of the definition of the order parameter one speaks usually of a long-range order. For example, CDW is characterized by a correlation function

$$
K(\mathbf{r})=\int \rho\left(\mathbf{r}_{0}\right) \rho\left(\mathbf{r}-\mathbf{r}_{0}\right) d V_{0},
$$

where the integration is performed over the elementary cell. The long-range order is determined by a nondecaying asymptotic behavior of the correlation function $K(\mathbf{r})$ at infinity

$$
\lim _{|\mathbf{r}| \rightarrow \infty} K(\mathbf{r}) \propto \cos (\mathbf{Q r})
$$

where $\mathbf{Q}$ is vector of the CDW oscillations. It is the longrange order that characterizes any crystalline structure unambiguously. 
Space and time play in many respects a similar role, and it looks quite natural to extend the notion of the space crystals to (thermodynamic) time-space crystals just adding an additional time coordinate to the above definitions and using coordinates $R=(t, \mathbf{r})$ on equal footing. Suppose, one comes to an order parameter $B(R)$ oscillating both in time and space. Again, one should assume in analogy with Eq. (1.1) that

$$
\int B\left(R-R_{0}\right) d R_{0}=0,
$$

where $R_{0}=\left(t_{0}, \mathbf{r}_{0}\right)$. The integration in Eq. (1.4) is performed over the period in time-space along a primitive vector. Now the primitive vectors include the one directed along the time axis. The integral (1.4) is assumed to be zero for the integration along any primitive vector including integration over time.

It is clear that already averaging over $t_{0}$ must give zero (or a time-independent constant) if one speaks of a state in the thermodynamic equilibrium. Indeed, there cannot be any selected $t_{0}$ in the equilibrium, although a certain time $t_{0}$ appears naturally in non-equilibrium situations marking the beginning of a process. This fact can be emphasized explicitly by the following integral

$$
\int_{\text {period }} B\left(t-t_{0}, \mathbf{r}-\mathbf{r}_{0}\right) d t_{0}=0 .
$$

In Eq. 1.5 the integration is performed over the period of the function $B(t)$.

The long-range order both in space and time is introduced using the correlation function

$$
K(R)=\int B\left(-R_{0}\right) B\left(R-R_{0}\right) d \Omega_{0},
$$

where the integration is performed over the elementary cell in time-space. The long-range order in time-space is determined by the following asymptotic behavior

$$
\lim _{|\mathbf{r}| \rightarrow \infty,|t| \rightarrow \infty} K(R) \propto \cos (\mathbf{Q r}) \cos (\Gamma t),
$$

and $\Gamma$ is a characteristic energy.

Here, only macroscopic systems with the volume of the system $V \rightarrow \infty$ in the thermodynamic equilibrium are considered, and Eqs. 1.7 should be valid for arbitrarily large volumes and times. Both $\mathbf{Q}$ and $\Gamma$ in Eq. (1.7) are supposed to be independent on the volume $V \rightarrow \infty$. Oscillations in time of two-times correlation functions are very well known in, e.g., two-or more level systems. However, in the limit $V \rightarrow \infty$ the level spacing $\Delta$ in such systems goes to zero and the frequency of the oscillations vanishes, which contrasts Eq. 1.7 written for finite $\Gamma$ dependent on internal parameters of the model but not on the volume. The systems possessing these properties are classified here as 'thermodynamic quantum time-space crystals'. To the best of my knowledge, this type of behavior has not been known so far.
Although Eqs. 1.4 1.7 written in analogy with Eqs. 1.2 1.3 are just a guess, they, if accepted, can exclude certain types of proposals on how to realize the time crystals. Quantum-mechanical averaging of an operator can lead to classical oscillations of, e.g., currents, and this would result in a radiation and a loss of energy, which is not possible in a thermodynamically stable state. The energy can also be lost due radiation of phonons in solid, etc.. However, as follows from the present discussion, any time-dependent order parameter of a thermodynamically stable state can appear only in the form of $B\left(t-t_{0}, \mathbf{r}\right)$ with arbitrary $t_{0}$. Then, integrating over $t_{0}$ like it is done in Eq. 1.5 gives zero (or constant), and one cannot have anything like currents oscillating in time. (If the integral in Eq. 1.5 is a time-independent constant one can subtract the corresponding constant from the definition of $B(t, \mathbf{r}))$. All this means that Eq. 1.5 is a necessary condition for any model proposed for the thermodynamically stable time crystal, and the latter is an essentially quantum phenomenon. At the same time, Fourier transform of two-time correlation functions determines scattering amplitudes, and therefore there should be possibility to observe the thermodynamically stable time crystals experimentally.

It is important to emphasize that, in this introductory section, we merely want to discuss some possibilities of introducing the thermodynamic quantum time crystals (TQTC) without violating laws of the nature, and, at the same time, make this notion very similar to the space crystals. The discussion of this section is not necessary for performing explicit calculations within a model introduced later in the paper, and serves only for visualizing results in simple terms.

Several years ago Wilczek ${ }^{2]}$ has proposed a concept of quantum time crystals using a rather simple model that possessed a state with a current oscillating in time. However, a more careful consideration of the mode ${ }^{3}$ has led to the conclusion that this was not the equilibrium state. These publications were followed by a hot discussion of the possibility of realization of a thermodynamically stable quantum time crystal4 9 . More general arguments against thermodynamically stable quantum time crystals in a macroscopic system have been presented later ${ }^{10}$. As a result, a consensus has been achieved that thermodynamical macroscopic quantum time crystals could not exist.

Slowly decaying oscillations in systems out of equilibrium are not forbidden by the 'no-go' theorems, and their study is definitely interesting by its own. Recent theoretica $11-16$ and experimental $\frac{17}{19}$ works have clearly demonstrated that this research field is very interesting and is fast growing. At present, the term 'Quantum Time Crystal' is usually used for non-equilibrium systems. It is difficult to cite here all papers already published in this direction of research but this activity is clearly different from the investigation of the possibility of the thermodynamically stable time crystal presented below.

It comes as a great surprise that a time-space crystal 
may exist as a thermodynamically stable state 20 . We use the term 'Thermodynamic Quantum Time Crystal' (TQTC) here to distinguish between the equilibrium and out-of-equilibrium states.

It turns out that the TQTC state can appear as a result of the breaking of the time-translation invariance of the original model and formation of a time-dependenent order parameter $B(t)$ with the properties described by Eqs. (1.4 1.7). This is a new effect. Actually, the 'no-go' theorem ${ }^{I 0}$ is proven only for models where such a symmetry breaking does not occur and is definitely valid for conventional models considered in the past. However, the formation of the time-dependent order parameter invalidates the proof and this will be discussed in detail later.

In this paper, considering a model of interacting fermions it is demonstrated that the system can undergo a phase transition into a state with an order parameter oscillating in both imaginary $\tau$ and real $t$ time. Studying the behavior in imaginary time $\tau$ is necessary for calculation of the free energy of the system, which is a standard very convenient method in quantum field theory. The period of the oscillations in the imaginary time equals $1 / m T$, where $m$ is integer, as required by boundary conditions for bosonic fields. The phase of the oscillations is arbitrary and the average over the position both in real and imaginary time of the periodic structure equals zero, in agreement with Eqs. 1.4, 1.5). Therefore, the system does not lose energy, which is the necessary condition for the thermodynamic equilibrium. The correlation function of the order parameters at real times has the form of Eq. (1.7), and its Fourier-transform determines the quantum scattering cross section. The TQTC obtained here can exist in arbitrarily big volume and is a completely new type of ordered states of matter.

Although being rather general, the model considered here has been introduced previously in a slightly different form of spin-fermion model with overlapping hot spots (SFMOHS) for description of underdoped superconducting cuprates 21 . The new state of TQTC obtained within this model is characterized by a loop currents order parameter oscillating both in space and time. The phase of the oscillations in time is arbitrary and averaging over the latter gives zero. As a result, the time reversal symmetry is broken but no static magnetic moments appear. These features may correspond to the pseudogap state $^{24} \sqrt{26}$ and we make explicit calculations and obtain results having in mind this possibility.

\section{B. Pseudogap state in superconducting cuprates.}

The pseudgap state is characterized by the loss of density of states due to the opening of a partial gap at the Fermi level below the pseudogap temperature $T^{*}>T_{c}$, where $T_{c}$ is the superconducting temperature. This gap decreases monotonously with the hole doping, which has been first observed in NMR (Knight shift) ${ }^{27 / 28}$ and, more recently, ARPES ${ }^{26 \mid 29]}$ and Raman ${ }^{30 \mid 31]}$ scattering studies.

However, modern experiments add a lot of unconventional details to this picture, showing that various ordering tendencies play a crucial role in the pseudogap state. The point-group symmetry of the $\mathrm{CuO}_{2}$ planes is broken in the pseudogap phase, which is seen from scanning tunneling microscopy (STM) $)^{32 \mid 33}$ and transport studies $34 \sqrt{35}$ of the pseudogap phase. More recently, magnetic torque measurements ${ }^{36}$ of the bulk magnetic susceptibility confirmed $C_{4}$ breaking occurring at $T^{*}$. Additionally, an inversion symmetry breaking associated with pseudogap has been discovered by means of second harmonic optical anisotropy measurement 37 .

Other experiments suggest that an unconventional time-reversal symmetry breaking can also be associated with the pseudogap. Polarized neutron diffraction studies of different cuprate families reveal a magnetic signal commensurate with the lattice appearing below $T^{*}$ and interpreted as being due to a $\mathbf{Q}=0$ intra-unit cell magnetic order 38 . The signal has been observed to start developing above $T^{*}$ with a finite correlation length $\underline{40 \mid 41}$ and breaks the $C_{4}$ symmetry of such a signal. Additionally, at a temperature $\mathrm{Tk}$ that is below Tc but shares a similar doping dependence, polar Kerr effect has been observed ${ }^{4243}$, which indicates ${ }^{4445}$ that time-reversal symmetry is broken. Additional signatures of a temporally fluctuating magnetism below $T^{*}$ are also available from the recent $\mu \mathrm{Sr}$ studies ${ }^{46}$.

While the signatures described above indicate that the pseudogap is a distinct phase with a lower symmetry, there also exist experiments ${ }^{24 \mid 47}$ with thermodynamic evidence for a corresponding phase transition. Transport measurements suggest the existence of quantum critical points (QCPs) of the pseudogap phase ${ }^{48}$, accompanied by strong mass enhancement ${ }^{49}$ in line with the existence of a $\mathrm{QCP}$.

As for theory, one of the initial interpretations was that the pseudogap is a manifestation of a fluctuating superconductivity, either in a form of preformed Bose pairs $50 / 51$ or strong phase fluctuations 52 .

However, the onset temperatures of superconducting fluctuations observed in experiments 53154 are considerably below $T^{*}$ and have a distinct doping dependence. Another scenario dating back to Ref 55 attributes the pseudogap to strong short-range correlations due to a strong on-site repulsion. However, this scenario does not explain the broken symmetries of the pseudogap state.

A different class of proposals for explaining the pseudogap involves a competing symmetry-breaking order. One of the possible candidates discussed in the literature is a $\mathbf{Q}=0$ orbital loop current order $\sqrt[56]{58}$. While successfully describing some of the experimentally observed phenomena, in particular, those of Refs. $\frac{38}{41}$, it does not lead to a gap on the Fermi surface at the mean-field level. Numerical studies of the three-band Hubbard model give arguments both for $\frac{5960}{60}$ and against $61 \sqrt[63]{63}$ this type of order. Moreover, in contrast to the experiments 48 the loop currents at $\mathbf{Q}=0$ have not been observed in recent 
experiments 64 on neutron scattering, which is in agreement with negative results of experiments on nuclear magnetic resonance and spin rotation measurements.

More recent studies focused on the important role of the interplay between CDW and superconducting fluctuations $\sqrt{65}$, preemptive orders 6 , CDW phase fluctuations 67, and hypothetical $\mathrm{SU}(2)$ symmetry $68 \mid 69$. Breaking of the time reversal and $C_{4}$ symmetries is not easily obtained from those models, though.

An interesting possibility is the d-density wave (DDW) state (also known as orbital flux phase ${ }^{71 / 72}$ ) which is characterized by a pattern of bond currents modulated with the wavevector $\mathbf{Q}=(\pi, \pi)$ and is not generally accompanied by a charge modulation. This order leads to a reconstructed Fermi surface consistent with the transport $\frac{4873}{173}$ and ARPES signatures of the pseudogap. Moreover, the time-reversal symmetry is also broken and a modified version of DDW can explain the polar Kerr effect ${ }^{74}$ observation. Additionally, model calcula-

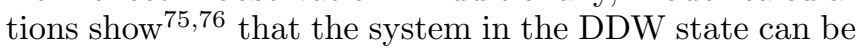
unstable to the formation of axial CDWs.

Experimental studies ${ }^{77 / 78}$ aimed at direct detection of magnetic moments created by the DDW state seemed to give results in favor of the existence of the magnetic moments. At the same time, neutron scattering 79 $\mu S R^{80}$ experiments have rather unambiguously demonstrated absence of any static magnetic order at $\mathbf{Q}=$ $(\pi, \pi)$. At present, there is no clear evidence for existence in the cuprates of static magnetic moments corresponding to the DDW.

In principle, the idea of using the DDW state for explanation of the origin of the pseudogap state might look promising 70 because many observed effects correlate with predictions of this proposal (see, e.g. Refs. $\frac{74|81| 82}{}$ ). At the same time, as already mentioned, a magnetic structure with the vector $\mathbf{Q}=(\pi, \pi)$ has not really been confirmed experimentally, although a time dependent magnetism was seen recently ${ }^{46}$ below $T^{*}$.

If the pseudogap state really corresponded to a temporarily oscillating loop currents order parameter with $\mathbf{Q}=(\pi, \pi)$, the magnetic moments would not be seen in neutron elastic scattering experiments. At the same time, the time reversal symmetry would be broken with all consequences and the gap in the spectrum would exist in the antinodal regions. The goal of this paper is to show that the TQTC state leading to such a picture is really possible.

\section{Plan of the presentation.}

In this paper we will demonstrate considering a spinfermion model that the TQTC can exist and the properties of the order parameter correspond to those written in Eqs. 1.4 1.7. It has been shown previously 23 that the model under consideration can give the DDW state. Now we will show that the model allows one to obtain a state with zero static loop currents but non-zero dynamic correlation of the currents that can be observed in, e.g., inelastic spin-polarized neutron scattering. At the same time, many other properties are similar to those of the DDW state.

In Section [I] a spin-fermion model with overlapping hot spots is introduced and simplified, in Section III general formulas for the partition function are derived decoupling of the electron-electron interaction by integration over auxiliary fields and minimizing a free energy functional containing these fields, while in Section IV the free energy of the system is calculated. Section $\mathrm{V}$ is devoted to calculation of time-dependent correlation functions using averaging over phases of oscillations, while in Section VI the same correlation functions are calculated using an operator order parameter and a quantum mechanical averaging. In Section VII the main results are re-derived using the Hamiltonian formulation of the model and the disagreement with the 'no-go' theorem is explained. In Section VIII possibilities of experimental observation of the 'Thermodynamic Qauntum Time-Space Crystal' are discussed, and the final discussion of the results is presented in Section IX.

\section{SPIN-FERMION MODEL WITH OVERLAPPING HOT SPOTS (SFMOHS) AND ITS SIMPLIFICATION.}

The spin-fermion model with overlapping hot spots (SFMOHS) has been suggested and further studied in Refs. 2123 for description of superconducting cuprates. This model originates from previously used spin-fermion models with 8 hot spots $65|66| 68|83| 84$ by the assumption that the hot spots on the Fermi surface are not isolated, may overlap and form antinodal 'hot regions'. This can happen when the fermion energies are not far away from the van Hove singularities in the spectrum of the cuprates, which corresponds to results of ARPES study 4385.87.

It is assumed that most important are momenta near the middles of the edges of the Brillouin zone (hot regions) and the latter are numerated as 1 and 2 (see Fig. 1 a).

The vector $\mathbf{Q}_{A F}$ connecting the middles of the edges equals the vector of the antiferromagnetic modulation of the parent compound. This contrasts the structure of the 'conventional' spin-fermion model with 8-hot spots $65|66| 68|83| 84$.

We write the partition function $Z$ of the system in a form of a functional integral over anticommuting vector fields $\chi_{\alpha}^{a}(X)$ with the subscript $\alpha$ and the superscript $a$ numerating spin and the hot regions

$$
Z=\int \exp \left[-S_{0}[\chi]-S_{\text {int }}[\chi]-S_{\mathrm{c}}[\chi]\right] D \chi .
$$

As usual, the fields $\chi$ satisfy the fermionic boundary conditions

$$
\chi(\tau)=-\chi(\tau+1 / T) .
$$




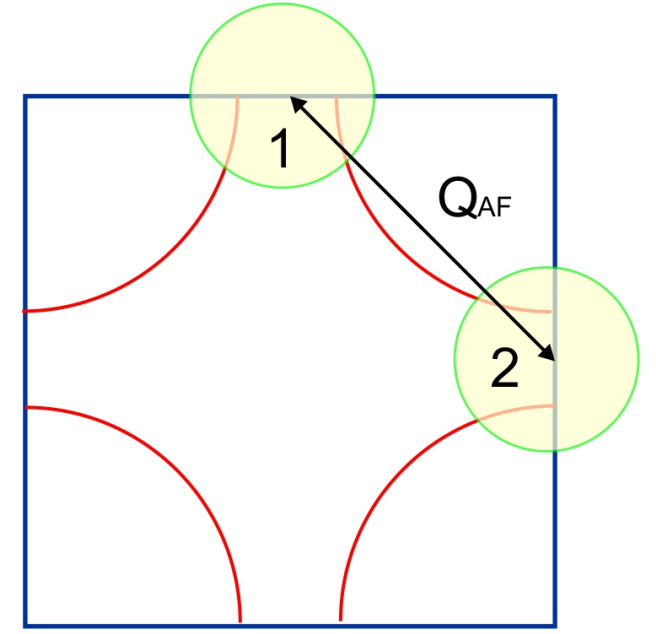

(a) Fermi surface and interaction.

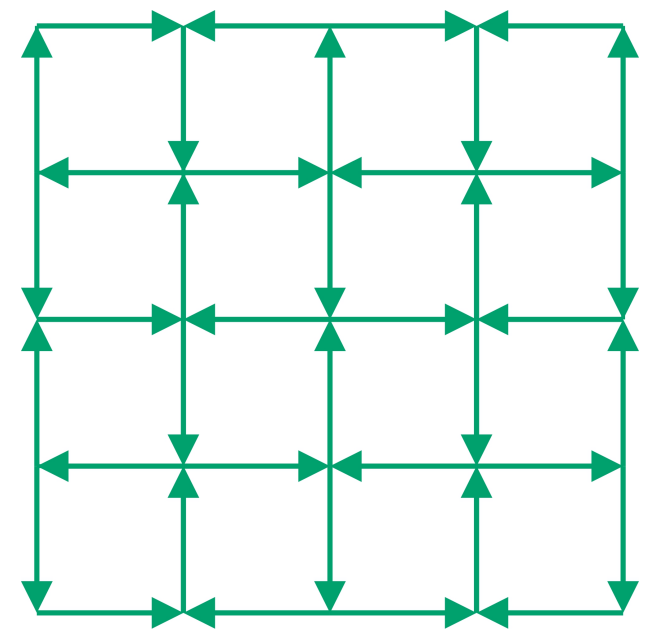

(b) Loop currents.

FIG. 1: (Color online.) Spin-fermion model with overlapping hot spots and loop currents.

In Eq. 2.1, the action $S_{0}$ of the non-interacting particles equals

$S_{0}=\int \chi^{+}(X)\left[\partial_{\tau}+\varepsilon^{+}(-i \nabla)+\Sigma_{3} \varepsilon^{-}(-i \nabla)\right] \chi(X) d X$

Herein, vectors $\chi$ and $\chi^{+}$have components

$$
\chi=\left(\begin{array}{c}
\chi_{1}^{1} \\
\chi_{2}^{1} \\
\chi_{1}^{2} \\
\chi_{1}^{2}
\end{array}\right), \quad \chi^{+}=\left(\begin{array}{llll}
\chi_{1}^{1 *} & \chi_{2}^{1 *} & \chi_{1}^{2 *} & \chi_{2}^{* 2}
\end{array}\right)
$$

and $X=(\tau, \mathbf{r})$ is a 4-dimensional coordinate in space and imaginary time $\tau$, varying in the interval $0<\tau<1 / T$, $T$ is temperature. In Eq. 2.3

$$
\varepsilon^{ \pm}(\mathbf{p})=\frac{1}{2}\left(\varepsilon_{1}(\mathbf{p}) \pm \varepsilon_{2}(\mathbf{p})\right),
$$

where $\varepsilon_{1}(\mathbf{p})$ and $\varepsilon_{2}(\mathbf{p})$ are two-dimensional spectra of the fermions in the regions 1 and 2 counted from the chemical potential $\mu$ (momenta are counted from the middles of edges). Matrices $\Sigma_{1}, \Sigma_{2}, \Sigma_{3}$ are Pauli matrices in the space of numbers 1 and 2 numerating the hot regions.

The interaction via antiferromagnetic paramagnons reads

$$
\begin{aligned}
& S_{\text {int }}\left[\chi, \chi^{+}\right]=-\frac{\lambda^{2}}{2} \int D_{0}\left(X-X^{\prime}\right) \\
& \times\left(\chi^{+}(X) \vec{\sigma} \Sigma_{1} \chi(X)\right)\left(\chi^{+}\left(X^{\prime}\right) \vec{\sigma} \Sigma_{1} \chi\left(X^{\prime}\right)\right) d X d X^{\prime},
\end{aligned}
$$

where $\lambda$ is a coupling constant, and $D_{0}$ is propagator of critical paramagnons. Its Fourier transform can be written as

$$
D_{0}(\omega, \mathbf{q})=\left(\omega^{2} / v_{s}^{2}+\mathbf{q}^{2}+\xi^{-2}\right)^{-1}
$$

where $\xi$ is a correlation length characterizing proximity to the antiferromagnetic transition point and $v_{s}$ is the velocity of antiferromagnetic excitations. The term $S_{\text {int }}[\chi]$ describes the interaction between the fermions of the region 1 and 2 .

We concentrate on more energetically favorable singlet electron-hole pairings between the regions 1 and 2. This allows one to replace the action $S_{\text {int }}[\chi]$ by the following effective action

$$
S_{\text {int }}\left[\chi, \chi^{+}\right] \rightarrow S_{\text {int }}^{\text {(current) }}\left[\chi, \chi^{+}\right]+S_{\text {int }}^{\text {(density) }}\left[\chi, \chi^{+}\right],
$$

where

$$
\begin{aligned}
& S_{\text {int }}^{\text {(current) }}\left[\chi, \chi^{+}\right]=-\frac{3 \lambda^{2}}{8} \int D_{0}\left(X-X^{\prime}\right) \\
& \times\left(\chi^{+}\left(X^{\prime}\right) \Sigma_{2} \chi(X)\right)\left(\chi^{+}(X) \Sigma_{2} \chi\left(X^{\prime}\right)\right) d X d X^{\prime},
\end{aligned}
$$

$$
\begin{aligned}
& S_{\text {int }}^{\text {(density) }}\left[\chi, \chi^{+}\right]=\frac{3 \lambda^{2}}{8} \int D_{0}\left(X-X^{\prime}\right) \\
& \times\left(\chi^{+}\left(X^{\prime}\right) \Sigma_{1} \chi(X)\right)\left(\chi^{+}(X) \Sigma_{1} \chi\left(X^{\prime}\right)\right) d X d X^{\prime} .
\end{aligned}
$$

In addition to the interaction via antiferromagnetic paramagnons, a $\mathbf{Q}_{A F^{-}}$-component term $S_{\mathrm{c}}\left[\chi, \chi^{+}\right]$of the 
Coulomb interaction has been added in equation 2.1

$$
\begin{aligned}
& S_{\mathrm{c}}\left[\chi, \chi^{+}\right]=\frac{1}{2} \int V_{\mathrm{c}}\left(X-X^{\prime}\right) \\
& \times\left(\chi^{+}(X) \Sigma_{1} \chi(X)\right)\left(\chi^{+}\left(X^{\prime}\right) \Sigma_{1} \chi\left(X^{\prime}\right)\right) d X d X^{\prime},
\end{aligned}
$$

which is very similar to $S_{\text {int }}^{\text {(density) }}[\chi]$ and does not give a contribution of the form of $S_{\text {int }}^{\text {(curent) }}[\chi]$, Eq. 2.9. Actually, Eq. $(2.9)$ describes an attraction of the loop currents, while Eqs. 2.10, 2.11) stand for a repulsion of the $(\pi, \pi)$ component of the charges.

The limit of the overlapping hot spots allows a variety of electron-hole as well as superconducting pairings ${ }^{23}$. In the model described by Eqs. 2.8, 2.11) several different phases have been identified within mean field schemes. This includes, depending on the parameters of the model, d-wave superconductivity, Pomeranchuk deformation of the Fermi surface, d-formfactor chargedensity waves with modulation vectors parallel to the bonds and d-density wave (DDW) state (loop currents). It is relevant to emphasize that all relevant energies like gaps in the spectrum, the Fermi energy, chemical potential, energy of the Pomeranchuk deformation of the Fermi surface, etc., are of the same order of magnitude. This corresponds to the experiments showing that all relevant energies of the various states are of order of several hundreds Kelvin, which is much smaller than usual electronic energies of the order of $1 \mathrm{eV}$.

Taking into account only the attraction term, Eq. 2.9 , one obtains ${ }^{23}$ the DDW state with the static currents represented in Fig. 1 b. The loop currents obtained in SFMOHS flow along the bonds of the square lattice and their direction is shown by arrows. The period of the oscillations equals the double period of the lattice. Within this picture one can speak of static magnetic moments oscillating in space.

Although using SFMOHS with the interaction specified by Eqs. 2.6 2.11 one can explicitly make calculations of various physical quantities for static order parameters, it is worth further simplifying the form of the interaction when investigating the possibility of formation of TQTC. This allows one to avoid unnecessary complications in calculations but, after all, the main issue of the present work is to demonstrate that TQTC is a general phenomenon, and keeping a detailed form of the interaction is not helpful for achieving this goal.

One comes to the simplified version of the model replacing the interactions $D_{0}\left(X-X^{\prime}\right)$ and $V_{\mathrm{c}}\left(X-X^{\prime}\right)$ by $\delta$-functions in both space and imaginary time

$$
\begin{aligned}
3 \lambda^{2} D_{0}\left(X-X^{\prime}\right) & \rightarrow 2 U_{0} \delta\left(X-X^{\prime}\right), \\
2 V_{\mathrm{c}}\left(x-X^{\prime}\right) & \rightarrow U_{\mathrm{c}} \delta\left(X-X^{\prime}\right) .
\end{aligned}
$$

The main results of this paper are derived using a scheme equivalent to a mean field approximation. Therefore, we start with a simplified model already adopted for using this scheme (the two-particle interaction contains squares of the sums over momenta and spins). We write such a simplified action $S\left[\chi, \chi^{+}\right]$in the form

$$
\begin{aligned}
& S\left[\chi, \chi^{+}\right]=S_{0}\left[\chi, \chi^{+}\right] \\
& -\frac{U_{0}}{4 V} \int_{0}^{1 / T}\left(\sum_{p} \chi_{p}^{+}(\tau) \Sigma_{2} \chi_{p}(\tau)\right)^{2} d \tau \\
& +\frac{\tilde{U}_{0}}{4 V} \int_{0}^{1 / T}\left(\sum_{p} \chi_{p}^{+}(\tau) \Sigma_{1} \chi_{p}(\tau)\right)^{2} d \tau,
\end{aligned}
$$

where

$$
\begin{aligned}
& S_{0}\left[\chi, \chi^{+}\right] \\
= & \int_{0}^{1 / T} \chi_{p}^{+}(\tau)\left[\partial_{\tau}+\varepsilon^{+}(\mathbf{p})+\Sigma_{3} \varepsilon^{-}(\mathbf{p})\right] \chi_{p}(\tau) d \tau .
\end{aligned}
$$

and

$$
\tilde{U}_{0}=U_{0}+U_{\mathrm{c}}>U_{0}
$$

It is assumed that $U_{0}>0, p=\{\mathbf{p}, \alpha\}$ stands for the momentum $\mathbf{p}$ and spin $\alpha$, and $V$ is the volume of the system.

The interaction terms correspond to a long-range coherence of pairs composed of electrons and holes belonging to the different bands but the interaction between the electrons is a short-range one.

The combination $\sum_{p} \chi_{p}^{+}(\tau) \Sigma_{1} \chi_{p}(\tau)$ stands for the $(\pi, \pi)$ charge oscillation, while $\sum_{p} \chi_{p}^{+}(\tau) \Sigma_{2} \chi_{p}(\tau)$ describes loop currents, Fig. 1b.

Action Eq. 2.13 written for the electron-hole pairs is similar to the one in the Bardeen-Cooper-Schrieffer (BCS) model for Cooper pairs ${ }^{88}$. It contains an interband attraction (term with matrix $\Sigma_{2}$ ) and repulsion (term with $\Sigma_{1}$ ). Taking into account only the term with the attraction one obtains an order parameter $B$ corresponding in the language of SFMOHS to spontaneous static loop currents, Fig. 1b. In order to obtain the new interesting state with a time-dependent order parameter one should consider both the interactions. It is crucial that the term with $\Sigma_{1}$ in Eq. 2.13 describing the interaction of charges is repulsive. The correspondence of action, Eq. 2.13), and the BCS model could be achieved formally putting $\tilde{U}_{0}=-U_{0}$ but this would contradict to the assumption 2.15). This is the reason why the results obtained in the present paper cannot be applied to the BCS superconductors.

\section{PARTITION FUNCTION AND EQUATIONS FOR THE MINIMUM OF A FREE ENERGY FUNCTIONAL OF BOSON FIELDS.}

\section{A. General formulas for the partition function.}

The order parameter of the model determined by the action 2.13 can be either static or oscillating both in 
real and imaginary time. Although the properties of the TQTC, Eqs. 1.4 1.7), are expected to follow from the real-time dependence of the order parameter, thermodynamics is determined by its imaginary-time behavior.

Now we write the partition function $Z$, Eq. (2.1), as

$$
Z=\int \exp \left[-S\left[\chi, \chi^{+}\right]\right] D \chi,
$$

where the action $S\left[\chi, \chi^{+}\right]$is specified by Eqs. 2.13, 2.14, and following the standard mean field theory introduce order parameters $b(\tau)$ and $b_{1}(\tau)$. Making a rotation of the fields $\chi$ in the space of numbers 1 and 2 of the bands

$$
\chi_{p}(\tau)=\mathcal{U}_{0} \eta_{p}(\tau), \quad \mathcal{U}_{0}=\frac{1}{\sqrt{2}}\left(\begin{array}{cc}
1 & i \\
i & 1
\end{array}\right)
$$

and using the relations

$$
\mathcal{U}_{0}^{+} \Sigma_{2} \mathcal{U}_{0}=\Sigma_{3}, \mathcal{U}_{0}^{+} \Sigma_{3} \mathcal{U}_{0}=-\Sigma_{2}, \quad \mathcal{U}_{0}^{+} \Sigma_{1} \mathcal{U}_{0}=\Sigma_{1},
$$

we write the action $S\left[\chi, \chi^{+}\right]$in terms of the anticommuting variables in the form

$$
S[\eta]=S_{0}[\eta]+S_{\mathrm{int}}[\eta],
$$

where

$$
S_{0}[\eta]=\int_{0}^{1 / T} \eta_{p}^{+}(\tau)\left(\partial_{\tau}+\varepsilon^{+}(\mathbf{p})-\varepsilon^{-}(\mathbf{p}) \Sigma_{2}\right) \eta_{p}(\tau) d \tau,
$$

and

$$
\begin{aligned}
& S_{\text {int }}[\eta]=-\frac{1}{4 V} \int_{0}^{1 / T}\left[U_{0}\left(\sum_{p} \eta_{p}^{+}(\tau) \Sigma_{3} \eta_{p}(\tau)\right)^{2}\right. \\
& \left.-\tilde{U}_{0}\left(\sum_{p} \eta_{p}^{+}(\tau) \Sigma_{1} \eta_{p}(\tau)\right)^{2}\right] d \tau .
\end{aligned}
$$

Now we use a method of integration over auxiliary bosonic fields $b(\tau)$ and $b_{1}(\tau)$ (Hubbard-Stratonovich transformation) to decouple the interaction in $S_{\text {int }}(\eta)$, Eq. (3.6)

$$
\begin{aligned}
& \exp \left[-S_{\text {int }}[\eta]\right]=\int \exp \left[-\int_{0}^{1 / T}\left[\frac{b^{2}(\tau)}{U_{0}}+\frac{b_{1}^{2}(\tau)}{\tilde{U}_{0}}\right] d \tau\right] \\
& \times Z_{b}^{-1} \exp \left[\int_{0}^{1 / T} \eta_{p}^{+}\left(b(\tau) \Sigma_{3}+i b_{1}(\tau) \Sigma_{1}\right) d \tau\right] D b D b_{1},
\end{aligned}
$$

where

$$
Z_{b}=\int D b D b_{1} \exp \left[-\int_{0}^{1 / T}\left[\frac{b^{2}(\tau)}{U_{0}}+\frac{b_{1}^{2}(\tau)}{\tilde{U}_{0}}\right] d \tau\right] .
$$

The fields $b(\tau)$ and $b_{1}(\tau)$ must obey the bosonic boundary conditions

$$
b(\tau)=b(\tau+1 / T), \quad b_{1}(\tau)=b_{1}(\tau+1 / T) .
$$

Substituting Eq. 3.7 into Eqs. 3.6 3.4 we obtain an effective action quadratic in $\eta$, which allows us to integrate exactly over $\eta, \eta^{+}$. As a result, we represent the free energy $F$ in the form

$$
F=-T \ln \left[\int \exp \left[-\frac{\mathcal{F}\left[b, b_{1}\right]}{T}\right] D b D b_{1}\right] .
$$

Herein, the free energy functional $\mathcal{F}\left[b, b_{1}\right]$ equals

$$
\begin{aligned}
\frac{\mathcal{F}\left[b, b_{1}\right]}{T}= & \int_{0}^{1 / T}\left[-2 \sum_{\mathbf{p}} \operatorname{tr}\left[\ln \left(h(\tau, \mathbf{p})-i b_{1}(\tau) \Sigma_{1}\right)\right]_{\tau, \tau}\right. \\
& \left.+V\left(\frac{b^{2}(\tau)}{U_{0}}+\frac{b_{1}^{2}(\tau)}{\tilde{U}_{0}}\right)\right] d \tau
\end{aligned}
$$

where

$$
h(\tau, \mathbf{p})=\partial_{\tau}+\varepsilon^{+}(\mathbf{p})-\varepsilon^{-}(\mathbf{p}) \Sigma_{2}-b(\tau) \Sigma_{3},
$$

and symbol 'tr' means trace in the space of the bands 1,2 .

Both the terms in Eq. (3.10) are proportional to the volume $V$ of the system, and therefore the saddle-point method becomes exact for calculation of the functional integral over the fields $b(\tau)$ and $b_{1}(\tau)$ in the limit $V \rightarrow$ $\infty$. In other words, the fields $b(\tau)$ and $b_{1}(\tau)$ should be found from the condition of the minimum of the free energy functional $\mathcal{F}\left[b, b_{1}\right]$, Eq. (3.10). Substitution of the functions obtained in this way into Eqs. (3.10, 3.9) gives the free energy $F$.

\section{B. Equations for the minimum of the free energy functional $\mathcal{F}\left[b, b_{1}\right]$ and their solutions at $b_{1}(\tau)=0$.}

Minimization of the free energy functional $\mathcal{F}\left[b, b_{1}\right]$, Eq. (3.10), leads to the following equations for $b(\tau)$ and $b_{1}(\tau)$

$$
\begin{gathered}
b(\tau)=-U_{0} \operatorname{tr} \int \Sigma_{3}\left[H^{-1}(\tau, \mathbf{p})\right]_{\tau, \tau} \frac{d \mathbf{p}}{(2 \pi)^{2}}, \\
b_{1}(\tau)=-i \tilde{U}_{0} \operatorname{tr} \int \Sigma_{1}\left[H^{-1}(\tau, \mathbf{p})\right]_{\tau, \tau} \frac{d \mathbf{p}}{(2 \pi)^{2}},
\end{gathered}
$$

where

$$
H(\tau, \mathbf{p})=h(\tau, \mathbf{p})-i b_{1}(\tau) \Sigma_{1} .
$$

Equations 3.12 3.13 have a time-independent solution ${ }^{23}$

$$
b_{1}(\tau)=0, b(\tau)=\gamma,
$$

where $\gamma$ determines the gap in the spectrum and can be bound from the equation

$$
1=\frac{U_{0}}{2} \int \frac{\tanh \frac{\kappa_{\mathbf{p}}^{(0)}+\varepsilon^{+}(\mathbf{p}) / T}{2}+\tanh \frac{\kappa_{\mathbf{p}}^{(0)}-\varepsilon^{+}(\mathbf{p}) / T}{2}}{\sqrt{\left(\varepsilon^{-}(\mathbf{p})\right)^{2}+\gamma^{2}}} \frac{d \mathbf{p}}{(2 \pi)^{2}},
$$


and

$$
\kappa_{\mathbf{p}}^{(0)}=\frac{\sqrt{\left(\varepsilon^{-}(\mathbf{p})\right)^{2}+\gamma^{2}}}{T} .
$$

Of course, the trivial solution $b_{1}(\tau)=0, b(\tau)=0$ also exists but we are interested in the region of parameters of the model where non-zero solutions appear.

Provided $\varepsilon^{+}(\mathbf{p})$ is not very large, Eq. 3.15 simplifies at low temperatures to the form

$$
1=U_{0} \int \frac{1}{\sqrt{\left(\varepsilon^{-}(\mathbf{p})\right)^{2}+\gamma^{2}}} \frac{d \mathbf{p}}{(2 \pi)^{2}} .
$$

However, more sophisticated time-dependent solutions of Eqs. 3.12, 3.13) also exist even at $\tilde{U}_{0}=0$. Although it was assumed in the beginning that $\tilde{U}_{0}>0$ (2.15) and this is the most interesting case, we start with formally considering the limit $\tilde{U}_{0}=0$ because it helps to understand the structure of the solutions at arbitrary $\tilde{U}_{0}$.

Setting $\tilde{U}_{0}=0$ in Eq. 3.13 and, hence $b_{1}(\tau)=0$, we come to the following equation for $b(\tau)$

$$
b(\tau)=-U_{0} \operatorname{tr} \int \Sigma_{3}\left[h^{-1}(\tau, \mathbf{p})\right]_{\tau, \tau} \frac{d \mathbf{p}}{(2 \pi)^{2}},
$$

with the operator $h(\tau, \mathbf{p})$ specified by Eq. 3.11.

Although Eq. 3.18 is quite non-trivial due to a possible dependence of $b(\tau)$ on $\tau$, solutions $b_{0}(\tau)$ of this equations can be written exactly in terms of a Jacobi double periodic elliptic function sn $(x \mid k)$,

$$
b_{0}(\tau)=k \gamma \operatorname{sn}\left(\gamma\left(\tau-\tau_{0}\right) \mid k\right),
$$

where $0<k<1$ is the modulus, $\gamma$ is an energy, and $\tau_{0}$ is an arbitrary shift of the imaginary time in the interval $0<\tau_{0}<1 / T$ (see also Fig. 2). The period of the oscillations for an arbitrary $k$ equals $4 K(k) / \gamma$, where $K(k)$ is the elliptic integral of the first kind, and therefore the condition

$$
\gamma=4 K(k) m T
$$

with integer $m>0$ must be satisfied to fulfill Eqs. (3.8).

One can visualize the function $u=k \operatorname{sn}(x \mid k)$ satisfying the equation

$$
\left(\frac{d u}{d x}\right)^{2}=u^{4}-\left(1+k^{2}\right) u^{2}+k^{2}
$$

by taking into account the fact that it describes motion of a classical particle with unit mass and energy $k^{2} / 2$ in the potential $w=\frac{1}{2}\left(u^{2}\left(1+k^{2}\right)-u^{4}\right)$. In the limit $k \rightarrow 1$, the particle starts moving from the top a hill in Fig. $2 \mathrm{k}$, stops on the other hill, and then moves back forming an instanton-anti-instanton pair (IAP). In this limit, the function $u$ has a simple form of alternating functions $\tanh x$ and $-\tanh x$ (instantons and antiinstantons), and is represented in Fig. 2b. In the limit of small $k$, the Jacobi elliptic function has the asymptotic behavior $\operatorname{sn}(x \mid k) \rightarrow \sin x$ corresponding to a harmonic oscillation of the classical particle near a minimum of $w$.

At small $1-k$, the period $4 K(k) / \gamma$ of $b_{0}(\tau)$ grows logarithmically as $-\ln (1-k)$ and the solution $b_{0}(\tau)$ consists of $2 m$ well separated alternating instantons and anti-instantons. It is important that the integral over the period of the oscillations in Eq. 3.19 equals zero. The average over the position $\tau_{0}$ equals zero as well

$$
\overline{b_{0}(\tau)}=0,
$$

where bar stands for such an averaging.

The solution (3.19) of Eq. (3.18) satisfying the boundary condition, Eq. (3.20), has been used previously in Refs ${ }^{89190}$ starting from a different model. A similar solution for a mean field equation arising in 1D models of polymers (depending on coordinates but not on the imaginary time) has been discovered long agd ${ }^{92}$ and later used also in Refs ${ }^{93 / 94}$.

One can demonstrate that the function $b_{0}(\tau)$, Eq. (3.19), is really the solution of Eq. (3.18) by finding the eigenfunctions $\Psi_{s \mathbf{p}}^{(0)}(\tau)$ and eigenenergies $\epsilon_{s \mathbf{p}}^{(0)}$ of the operator $h(\tau, \mathbf{p})$, Eq. 3.11), with $b(\tau)=b_{0}(\tau)$, where $b_{0}(\tau)$ satisfies equation

$$
\dot{b}_{0}^{2}(\tau)=b_{0}^{4}(\tau)-\gamma^{2}\left(1+k^{2}\right) b_{0}^{2}(\tau)+\gamma^{4} k^{2},
$$

and making a spectral expansion of the operator $h^{-1}(t, \mathbf{p})$.

It is instructive to give details of the calculations because an extension of this formalism will be used for nonzero $b_{1}(\tau)$. At $b_{1}(\tau)=0$ we have equations

$$
\begin{aligned}
& h(\tau, \mathbf{p}) \Psi_{s \mathbf{p}}^{(0)}(\tau)=\epsilon_{s \mathbf{p}}^{(0)} \Psi_{s \mathbf{p}}^{(0)}(\tau), \\
& \bar{\Psi}_{s \mathbf{p}}^{(0)}(\tau) \bar{h}(\tau, \mathbf{p})=\epsilon_{s \mathbf{p}}^{(0)} \bar{\Psi}_{s \mathbf{p}}^{(0)}(\tau) .
\end{aligned}
$$

In Eqs. (3.24) operator $h(\tau, \mathbf{p})$ has been defined in Eq. (3.11) and $h(\tau, \mathbf{p})$ equals

$$
\bar{h}(\tau, \mathbf{p})=-\overleftarrow{\partial}_{\tau}+\varepsilon^{+}(\mathbf{p})-\varepsilon^{-}(\mathbf{p}) \Sigma_{2}-b_{0}(\tau) \Sigma_{3}
$$

where the derivative $\overleftarrow{\partial}_{\tau}$ acts on the left. The eigenfunctions $\bar{\Psi}_{s \mathbf{p}}^{(0)}(\tau)$ and $\Psi_{s \mathbf{p}}^{(0)}(\tau)$ have to obey the antiperiodic boundary conditions

$\Psi_{s \mathbf{p}}^{(0)}(\tau)=-\Psi_{s \mathbf{p}}^{(0)}(\tau+1 / T), \bar{\Psi}_{s \mathbf{p}}^{(0)}(\tau)=-\bar{\Psi}_{s \mathbf{p}}^{(0)}(\tau+1 / T)$

Solutions $\Psi_{s \mathbf{p}}^{(0)}(\tau), \bar{\Psi}_{s \mathbf{p}}^{(0)}(\tau)$ of Eqs. 3.24 satisfying Eqs. (3.26) can be sought in the form

$$
\begin{aligned}
& \Psi_{s \mathbf{p}}^{(0)}(\tau)=\mathcal{N}_{\mathbf{p}} \Upsilon_{l \mathbf{p}}(\tau) e^{-i \pi(2 n+1) \tau T} e^{-(-1)^{l} \kappa_{\mathbf{p}} T \tau}, \quad(3.27) \\
& \bar{\Psi}_{s \mathbf{p}}^{(0)}(\tau)=(-1)^{l+1} \mathcal{N}_{\mathbf{p}} \bar{\Upsilon}_{l \mathbf{p}}(\tau) e^{i \pi(2 n+1) \tau T} e^{(-1)^{l} \kappa_{\mathbf{p}} T \tau},
\end{aligned}
$$

where $s=\{l, n\}, l=1,2, n=0, \pm 1, \pm 2 \ldots$ and $\mathcal{N}_{\mathbf{p}}$ is a normalization factor.

Functions $\Upsilon_{l \mathbf{p}}(\tau)$ equal

$$
\Upsilon_{l \mathbf{p}}(\tau)=\left(\begin{array}{c}
1 \\
\left(i \varepsilon^{-}(\mathbf{p})\right)^{-1}\left(-\partial_{\tau}+b_{0}(\tau)\right)
\end{array}\right) Z_{l \mathbf{p}}(\tau),
$$




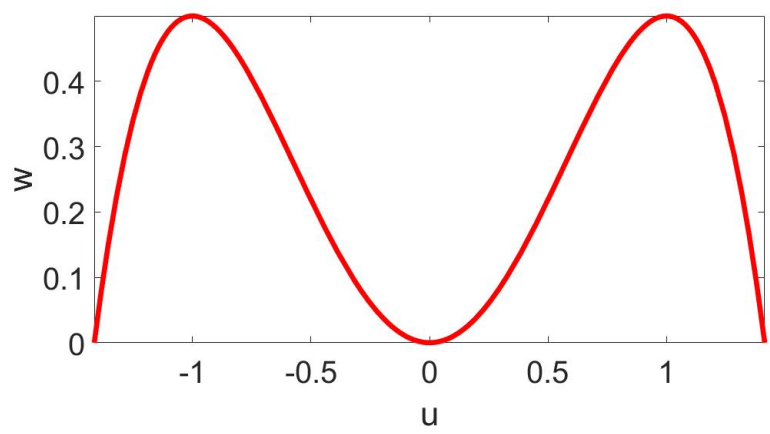

(a) Potential $w$ at $k=0.9999$.

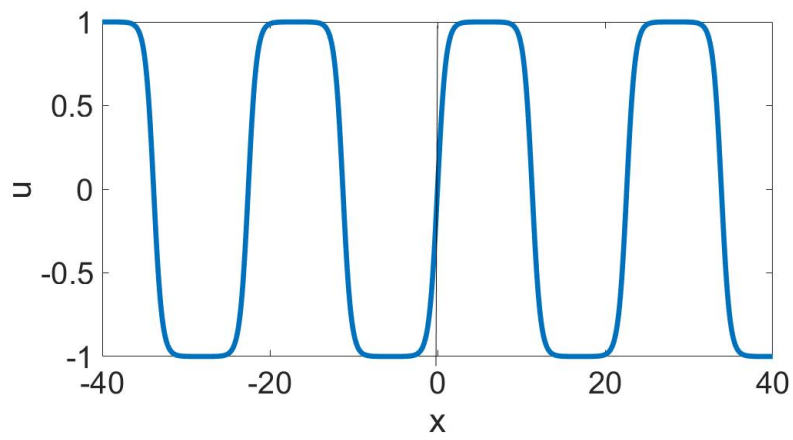

(b) Solution $u$ as a function of $x$ at $k=0.9999$

FIG. 2: (Color online.) Jacobi elliptic function.

$$
\bar{\Upsilon}_{l \mathbf{p}}(\tau)=\left(\left(i \varepsilon^{-}(\mathbf{p})\right)^{-1}\left(\partial_{\tau}-b_{0}(\tau)\right) 1\right) \bar{Z}_{l \mathbf{p}}(\tau),
$$

where

$\bar{Z}_{l}(\tau)=\left\{\begin{array}{cc}X_{\mathbf{p}}(\tau), & l=1 \\ Y_{\mathbf{p}}(\tau), & l=2\end{array}, \quad Z_{l}(\tau)=\left\{\begin{array}{cc}Y_{\mathbf{p}}(\tau), & l=1 \\ X_{\mathbf{p}}(\tau), & l=2\end{array}\right.\right.$,

and the functions $X_{\mathbf{p}}(\tau)$ and $Y_{\mathbf{p}}(\tau)$ are growing and decaying with $\tau$ solutions of the same equation

$$
\begin{gathered}
{\left[-\partial_{\tau}^{2}+\left(\varepsilon^{-}(\mathbf{p})\right)^{2}+Q(\tau)\right] Y_{\mathbf{p}}(\tau)=0} \\
{\left[-\partial_{\tau}^{2}+\left(\varepsilon^{-}(\mathbf{p})\right)^{2}+Q(\tau)\right] X_{\mathbf{p}}(\tau)=0}
\end{gathered}
$$

with

$$
Q(\tau)=b_{0}^{2}(\tau)+\dot{b}_{0}(\tau) .
$$

Although the functions $\Psi_{s \mathrm{p}}^{(0)}(\tau), \bar{\Psi}_{s \mathrm{p}}^{(0)}(\tau)$ obey the antiperiodicity conditions $(3.26)$, the solutions $X_{\mathbf{p}}(\tau)$ and $Y_{\mathbf{p}}(\tau)$ cannot be periodic. We assume that they change over the period $1 / T$ as

$X_{\mathbf{p}}(\tau+1 / T)=e^{\kappa_{\mathbf{p}}} X_{\mathbf{p}}(\tau), Y_{\mathbf{p}}(\tau+1 / T)=e^{-\kappa_{\mathbf{p}}} Y_{\mathbf{p}}(\tau)$,

where $\kappa_{\mathbf{p}}$ is a function of $\mathbf{p}$ only. The antiperiodicity of the eigenfunctions $\Psi_{s \mathbf{p}}^{(0)}(\tau), \bar{\Psi}_{s \mathbf{p}}^{(0)}(\tau)$ is guaranteed by the presence in Eq. (3.27) of the exponentials containing $\kappa_{\mathbf{p}}$. The solutions $X_{\mathbf{p}}(\tau)$ and $Y_{\mathbf{p}}(\tau)$ of Eqs. 3.31 are related to each other as

$$
\dot{X}_{\mathbf{p}}(\tau) Y_{\mathbf{p}}(\tau)-X_{\mathbf{p}}(\tau) \dot{Y}_{\mathbf{p}}(\tau)=C_{\mathbf{p}},
$$

where $C_{\mathbf{p}}$ is a time-independent function of $\mathbf{p}$.

Eqs. (3.27 3.34 are sufficient to prove the orthogonality of the eigenfunctions $\Psi_{s \mathbf{p}}^{(0)}(\tau)$

$$
\left(\bar{\Psi}_{s \mathbf{p}}, \Psi_{s^{\prime} \mathbf{p}}\right) \equiv T \int_{0}^{1 / T} \bar{\Psi}_{s \mathbf{p}}(\tau) \Psi_{s^{\prime} \mathbf{p}}(\tau) d \tau=\delta_{s s^{\prime}} .
$$

and write the normalization $\mathcal{N}_{\mathbf{p}}$ in the form

$$
\mathcal{N}_{\mathbf{p}}^{2}=\frac{i \varepsilon^{-}(\mathbf{p}) T}{C_{\mathbf{p}}} .
$$

Substituting Eqs. (3.27 3.30 into Eq. (3.24) one finds the eigenenergies $\epsilon_{s \mathbf{p}}^{(0)}$

$$
\epsilon_{s \mathbf{p}}^{(0)}=\varepsilon_{\mathbf{p}}^{+}(\mathbf{p})-i(2 n+1) \pi T+(-1)^{l+1} \kappa_{\mathbf{p}} T .
$$

The last step to be done for writing the eigenfunctions $\Psi_{s \mathbf{p}}^{(0)}(\tau), \bar{\Psi}_{s \mathbf{p}}^{(0)}(\tau)$ explicitly is to solve equations 3.31. The final solution for $X_{\mathbf{p}}(\tau)$ and $Y_{\mathbf{p}}(\tau)$ can be written in the form

$$
X_{\mathbf{p}}(\tau)=w_{\mathbf{p}}(\tau) \exp \left[\int_{0}^{\tau} \frac{\Omega_{\mathbf{p}}}{w_{\mathbf{p}}^{2}\left(\tau^{\prime}\right)} d \tau^{\prime}\right],
$$

$$
Y_{\mathbf{p}}(\tau)=w_{\mathbf{p}}(\tau) \exp \left[-\int_{0}^{\tau} \frac{\Omega_{\mathbf{p}}}{w_{\mathbf{p}}^{2}\left(\tau^{\prime}\right)} d \tau^{\prime}\right],
$$

where

$$
\begin{aligned}
& \Omega_{\mathbf{p}}=\left|\varepsilon^{-}(\mathbf{p})\right|^{3} \\
& \times \sqrt{\left(1+\frac{\gamma^{2}}{4} \frac{(1-k)^{2}}{\left(\varepsilon^{-}(\mathbf{p})\right)^{2}}\right)\left(1+\frac{\gamma^{2}}{4} \frac{(1+k)^{2}}{\left(\varepsilon^{-}(\mathbf{p})\right)^{2}}\right)},
\end{aligned}
$$

and

$$
w_{\mathbf{p}}(\tau)=\left(\left(\varepsilon^{-}(\mathbf{p})\right)^{2}+\frac{1+k^{2}}{4}-\frac{Q(\tau)}{2}\right)^{1 / 2} .
$$

These solutions can be checked substituting equations 3.38, 3.39 into Eqs. (3.31) and using Eq. 3.23). One can also see from equation (3.34) that

$$
C_{\mathbf{p}}=2 \Omega_{\mathbf{p}}
$$

and it is clear that the parameter $\kappa_{\mathbf{p}}$ equals

$$
\kappa_{\mathbf{p}}=\int_{0}^{1 / T} \frac{\Omega_{\mathbf{p}}}{w_{\mathbf{p}}^{2}(\tau)} d \tau
$$


One can derive from equations $3.28,3.30$ and $3.38,3.42$ the following useful relations

$$
\begin{array}{r}
\left(\bar{\Upsilon}_{l \mathbf{p}}(\tau) \Sigma_{3} \Upsilon_{l \mathbf{p}}(\tau)\right)=2 i b_{0}(\tau) \varepsilon^{-}(\mathbf{p}) \\
\left(\bar{\Upsilon}_{l \mathbf{p}}(\tau) \Sigma_{1} \Upsilon_{l \mathbf{p}}(\tau)\right)=-\dot{b}_{0}(\tau) .
\end{array}
$$

Using the spectral expansion of the function $h^{-1}(\tau, \mathbf{p})$ one can bring Eq. 3.18 to the form

$$
b(\tau)=-U_{0} \sum_{s} \frac{\bar{\Psi}_{s \mathbf{p}}^{(0)}(\tau) \Sigma_{3} \Psi_{s \mathbf{p}}^{(0)}(\tau)}{\epsilon_{s \mathbf{p}}^{(0)}}
$$

Substituting eigenfunctions $\Psi_{s \mathbf{p}}^{(0)}(\tau)$, Eqs. 3.273 .36 , and eigenenergies $\epsilon_{s \mathbf{p}}^{(0)}$, Eq. 3.37), into Eq. 3.46) we obtain finally the mean field equation for $b_{0}(\tau)$

$$
\begin{aligned}
& b_{0}(\tau)=\frac{U_{0}}{2} \int\left[\tanh \frac{\kappa_{\mathbf{p}}+\varepsilon_{\mathbf{p}}^{+} / T}{2}+\tanh \frac{\kappa_{\mathbf{p}}-\varepsilon_{\mathbf{p}}^{+} / T}{2}\right] \\
& \times \frac{b_{0}(\tau)\left|\varepsilon^{-}(\mathbf{p})\right|}{\sqrt{\left(\left(\varepsilon^{-}(\mathbf{p})\right)^{2}+\gamma^{2} \frac{(1-k)^{2}}{4}\right)\left(\left(\varepsilon^{-}(\mathbf{p})\right)^{2}+\gamma^{2} \frac{(1+k)^{2}}{4}\right)}} \frac{d \mathbf{p}}{(2 \pi)^{2}} .
\end{aligned}
$$

We see that $b_{0}(\tau)$ drops out from Eq. 3.47), and the latter is algebraic, which means that $b_{0}(\tau)$ is an exact solution of Eq. 3.18. Eq. 3.47 is valid for arbitrary temperature $T$ and modulus $k$. It should be solved together with Eq. 3.20 and one should find the solution for a given number $m$ of IAP. One has to calculate also the integral providing the value $\kappa_{\mathbf{p}}$, Eq. (3.43). Actually, it can be expressed in terms of the elliptic integrals of the first $K(\tilde{k})$ and third $\Pi(\tilde{k})$ kinds.

$$
\kappa_{\mathbf{p}}=\frac{\left|\varepsilon^{-}(\mathbf{p})\right|}{T} \sqrt{\frac{\left(\varepsilon^{-}(\mathbf{p})\right)^{2}+\frac{\gamma^{2}}{4}(1-k)^{2}}{\left(\varepsilon^{-}(\mathbf{p})\right)^{2}+\frac{\gamma^{2}}{4}(1+k)^{2}}} \frac{\Pi(n, \tilde{k})}{K(\tilde{k})}
$$

where

$$
n=\frac{\gamma^{2} k}{\left(\varepsilon^{-}(\mathbf{p})\right)^{2}+\frac{\gamma^{2}}{4}(1+k)^{2}}, \tilde{k}=\frac{2 \sqrt{k}}{1+k} .
$$

Equations (3.47,3.49) overlap with results of previous publications 8990 .

It is clear that at low temperatures there can be many solutions of Eq. (3.47) because it contains two unknown parameters $\gamma$ and $k$. The proper solution should correspond to the minimum of the free energy $\mathcal{F}\left[b, b_{1}\right]$, Eqs. 3.10 3.11. We will see that in the absence of the field $b_{1}(\tau)$, which is the case when the interaction $\tilde{U}_{0}$ in Eq. 3.13 is formally put to zero, the time-independent solution, Eq. (3.14), is most favorable. At non-zero $\tilde{U}_{0}$ one has to solve Eqs. 3.12, 3.13 together. Of course, this will modify the solution $b(\tau)$ but the solution alone cannot help answering the question whether configurations with a finite number of IAP can be energetically favorable or not. In other words, one has to calculate the free energy for a given number $m$ of IAP and check whether this energy can be lower than that with $m=0$ or not.

The calculations to be done look rather involved. Fortunately, the limit of a diluted system of IAP corresponding to a large period $(1-k \ll 1)$ is most favorable for obtaining a non-zero imaginary-time-dependent order parameter, and calculations become considerably simpler in this limit.

It is worth mentioning that, in the limit $1-k \ll 1$, Eq. 3.47), reduces to Eq. 3.15, and many thermodynamic properties of the diluted system of IAP can be very close to those of DDW state. At the same time, behavior of physical quantities like loop currents determined directly by the order parameter can be drastically different.

\section{FREE ENERGY.}

\section{A. General scheme of the calculations.}

In principle, the free energy $F$ should be calculated by first solving Eqs. $3.12,3.13$ for $b(\tau), b_{1}(\tau)$ for a given number $m$ of IAP and substituting the solution into the free energy functional $\mathcal{F}\left[b, b_{1}\right]$, Eqs. 3.10, 3.11. This would give a free energy $F[m]$ as a function of $m$. The minimum of this function is the free energy of the system.

This procedure resembles calculation of the free energy of vortices in a superconductor subjected to a magnetic $B$ field. In order to investigate the possibility of entering vortices into the superconductor one calculates the energy of a single vortex and the energy of its interaction with the magnetic field. The total energy is positive in the Meissner state, while it is negative in the mixed Abrikosov state. The energy equals zero at the critical field $B_{c 1}$. Even if it is not easy to find the precise form of the single vortex, the existence of the transition can be established in this way taking an approximate solution for the vortex.

Solving Eqs. 3.12, 3.13 exactly or investigating the problem numerically is not an easy task, and it is beyond the scope of the present work. Instead, we develop here an approximate scheme that provides physically plausible results. We proceed by introducing eigenfunctions $\Psi_{s \mathbf{p}}(\tau)$, its conjugates $\bar{\Psi}_{s \mathbf{p}}(\tau)$ and eigenenergies $\epsilon_{s \mathbf{p}}$, satisfying equations

$$
\begin{aligned}
& \left(h(\tau, \mathbf{p})-i b_{1}(\tau) \Sigma_{1}\right) \Psi_{s \mathbf{p}}(\tau)=\epsilon_{s \mathbf{p}} \Psi_{s \mathbf{p}}(\tau), \\
& \bar{\Psi}_{s \mathbf{p}}(\tau)\left(\bar{h}(\tau, \mathbf{p})-i b_{1}(\tau) \Sigma_{1}\right)=\epsilon_{s \mathbf{p}} \bar{\Psi}_{s \mathbf{p}}(\tau),
\end{aligned}
$$

and antiperiodicity conditions

$\Psi_{s \mathbf{p}}(\tau+1 / T)=-\Psi_{s \mathbf{p}}(\tau), \quad \bar{\Psi}_{s \mathbf{p}}(\tau+1 / T)=-\bar{\Psi}_{s \mathbf{p}}(\tau)$. $(4.2)$

Operators $h(\tau, \mathbf{p})$ and $\bar{h}(\tau, \mathbf{p})$ are specified in Eqs. 3.11 3.25. Functions $\Psi_{s \mathbf{p}}(\tau), \bar{\Psi}_{s \mathbf{p}}(\tau)$ form an orthogonal 
basis of the eigenfunctions

$$
\left(\bar{\Psi}_{s \mathbf{p}}, \Psi_{s^{\prime} \mathbf{p}}\right) \equiv T \int_{0}^{1 / T} \bar{\Psi}_{s \mathbf{p}}(\tau) \Psi_{s^{\prime} \mathbf{p}}(\tau) d \tau=\delta_{s s^{\prime}}
$$

Setting formally $b_{1}(\tau)=0$ one comes back to the eigenfunctions $\Psi_{s \mathbf{p}}^{(0)}(\tau), \bar{\Psi}_{s \mathbf{p}}^{(0)}(\tau)$ and eigenenergies $\epsilon_{s \mathbf{p}}^{(0)}$, Eqs. 3.243 .43 . Then, one can write the 'electronic' part $\mathcal{F}_{\text {el }}$ (first term in the integrand in Eq. (3.10) in the form

$$
\begin{aligned}
& \frac{\mathcal{F}_{\mathrm{el}}\left[b(\tau), b_{1}(\tau)\right]}{V T} \\
= & 2 \int_{0}^{1 / T} \sum_{\mathbf{p}} \operatorname{tr}\left[\ln \left(h(\tau, \mathbf{p})-i b_{1}(\tau) \Sigma_{1}\right)\right]_{\tau, \tau} d \tau \\
= & -2 \sum_{s} \int \ln \frac{\epsilon_{s \mathbf{p}}}{T} \frac{d \mathbf{p}}{(2 \pi)^{2}} .
\end{aligned}
$$

One should keep in mind that the eigenvalues $\epsilon_{s \mathbf{p}}$ are functionals of the functions $b(\tau)$ and $b_{1}(\tau)$. The fact that the functional $\mathcal{F}_{\text {el }}\left[b(\tau), b_{1}(\tau)\right]$ can be expressed in terms of only the eigenvalues simplifies calculations. We cannot find $\epsilon_{s \mathbf{p}}$ and $\Psi_{s \mathbf{p}}(\tau)$ exactly for arbitrary $b_{1}(\tau)$ and simply use a perturbation theory for the eigenvalues $\epsilon_{s \mathbf{p}}$. In the zeroth approximation one puts $b_{1}(\tau)=0$ and obtains the eigenvalues $\epsilon_{s \mathbf{p}}^{(0)}$, Eqs. 3.37, 3.48, 3.49.

As the next step, we assume non-zero $b_{1}(\tau)$ and write

$$
b(\tau)=b_{0}(\tau)+\delta b(\tau),
$$

where $b_{0}(\tau)$ is given by Eq. 3.19$)$. Then, we expand $\mathcal{F}_{\text {el }}\left[b(\tau), b_{1}(\tau)\right]$, Eq. 4.4 , in $b_{1}(\tau)$ and $\delta b(\tau)$ up to the second order in these variables. This will allows us to obtain an interaction between the fields $b(\tau)$ and $b_{1}(\tau)$ and take into account a screening of this interaction.

Although putting $b_{1}(\tau)=0$ leads to the correct static solution for $b$, it is generally not a good assumption because, as we will see, the field $b_{1}(\tau)$ linearly couples to the time derivative $\dot{b}_{0}(\tau)$ generating an additional term in the free energy of IAP

$$
\frac{\mathcal{F}_{\text {int }}\left[b_{1}\right]}{V T}=-\frac{J}{2} \int_{0}^{1 / T} \dot{b}_{0}(\tau) b_{1}(\tau) d \tau
$$

where $J$ is a constant.

Fluctuations of $b_{1}(\tau)$ generate an effective attraction between the instantons and anti-instantons and favor formation of $\tau$-dependent structures. Formally replacing $\tau$ by a space coordinate one can see that the mechanism of the attraction is similar to the one of the electronphonon interaction in solids. The field $b_{1}(\tau)$ plays in this picture the role of phonons and its fluctuations may result in a sufficiently strong attraction of instantons and anti-instantons and, eventually, lead to a function $b(\tau)$ oscillating in the imaginary time $\tau$.

The calculation of the free energy functional $\mathcal{F}_{\text {el }}\left[b(\tau), b_{1}(\tau)\right]$ is done by substituting

$$
\epsilon_{s \mathbf{p}}=\epsilon_{s \mathbf{p}}^{(0)}+\epsilon_{s \mathbf{p}}^{(1)}+\epsilon_{s \mathbf{p}}^{(2)},
$$

into Eq. 4.4 and calculating $\epsilon_{s \mathbf{p}}^{(1)}$ and $\epsilon_{s \mathbf{p}}^{(2)}$ with the help of standard quantum-mechanical formulas

$$
\begin{gathered}
\epsilon_{s \mathbf{p}}^{(1)}=-\int_{0}^{1 / T} \Pi_{s s}(\tau, \mathbf{p}) d \tau \\
\epsilon_{s \mathbf{p}}^{(2)}=\sum_{s^{\prime} \neq s} \int_{0}^{1 / T} \frac{\Pi_{s s^{\prime}}(\tau, \mathbf{p}) \Pi_{s^{\prime} s}(\tau, \mathbf{p})}{\epsilon_{s \mathbf{p}}^{(0)}-\epsilon_{s^{\prime} \mathbf{p}}^{(0)}} d \tau,
\end{gathered}
$$

where

$$
\Pi_{s s^{\prime}}(\tau, \mathbf{p})=\bar{\Psi}_{s \mathbf{p}}^{(0)}(\tau)\left(i b_{1}(\tau) \Sigma_{1}+\delta b(\tau) \Sigma_{3}\right) \Psi_{s^{\prime} \mathbf{p}}^{(0)}(\tau) .
$$

As soon as the electronic part is calculated, one should minimize $\mathcal{F}\left[b(\tau), b_{1}(\tau)\right]$, Eq. 3.10, 3.11, with respect to $b_{1}(\tau)$ and $\delta b(\tau)$, and calculate the free energy in terms of the solution $b_{0}(\tau)$, Eq. 3.19.

\section{B. Free energy for an arbitrary spectrum $\varepsilon^{-}(\mathbf{p})$.}

We consider here the most interesting limit of small $1-k \ll 1$ assuming that temperatures are low, $T \ll \gamma$. Taking $b_{0}(\tau)$ in the form of Eq. (3.19) one can determine the parameter $\gamma$ from Eq. 3.17) which is, at the same time, the mean field equation for the time-independent order parameter (gap in the electron spectrum) of the DDW state 23 . The modulus $k$ and, hence, the number of IAP drops out from Eq. 3.17) and can be determined only from the condition of the minimum of free energy. In the absence of the field $b_{1}(\tau)$, the minimum is always reached at the time-independent solution $b=\gamma$. The fields $b_{1}(\tau)$ couple to $\dot{b}_{0}(\tau)$, Eq. 4.6 , and a finite number $m$ of the instantons-antiinstantons can provide the absolute minimum of the free energy. This statement can be checked by calculating the free energy for finite $m$ in the linear approximation in this number. Negative values of the difference $\Delta F$ between this free energy and the free energy of the DDW will indicate the possibility of the imaginary-time-dependent state.

In the limit $1-k \ll 1$, one can write the parameter $\kappa_{\mathbf{p}}$ 3.48, 3.49 in a simplified form

$$
\kappa_{\mathbf{p}}=\kappa_{\mathbf{p}}^{(D D W)}+\kappa_{\mathbf{p}}^{(i n s t)},
$$

where

$$
\kappa_{\mathbf{p}}^{(D D W)}=\frac{\sqrt{\left(\varepsilon^{-}(\mathbf{p})\right)^{2}+\gamma^{2}}}{T}
$$

is the function $\kappa_{\mathbf{p}}$ for the DDW state, and

$$
\kappa_{\mathbf{p}}^{(i n s t)}=-m \ln \frac{1+\frac{\gamma}{\sqrt{\left(\varepsilon^{-}(\mathbf{p})\right)^{2}+\gamma^{2}}}}{1-\frac{\gamma}{\sqrt{\left(\varepsilon^{-}(\mathbf{p})\right)^{2}+\gamma^{2}}}}
$$

is the contribution of the instantons and antiinstantons. The term $\kappa_{\mathbf{p}}^{(\text {inst })}$ is smaller than $\kappa_{\mathbf{p}}^{(D D W)}$ at low temperatures but still can be large at large $m$. 
Eqs. 4.10 4.12 are used for calculation of $\epsilon_{s \mathbf{p}}^{(0)}$, Eq. (3.37). Substituting Eqs. 4.10 4.12 3.37 into 4.4, performing summation over $n$, and adding a contribution coming from the $b^{2}(\tau)$-term in Eq. 3.10 we obtain with the help of the self-consistency equation (3.17) the energy of $F_{\text {inst }}$ of 'non-interacting' instantons and antiinstantons

$$
\begin{aligned}
& \frac{F_{\text {inst }}}{2 m V T} \\
= & \int\left[\ln \frac{1+\frac{\gamma}{\sqrt{\left(\varepsilon^{-}(\mathbf{p})\right)^{2}+\gamma^{2}}}}{1-\frac{\gamma}{\sqrt{\left(\varepsilon^{-}(\mathbf{p})\right)^{2}+\gamma^{2}}}}-\frac{2 \gamma}{\sqrt{\left(\varepsilon^{-}(\mathbf{p})\right)^{2}+\gamma^{2}}}\right] \frac{d \mathbf{p}}{(2 \pi)^{2}} .
\end{aligned}
$$

The energy $F_{\text {inst }}$, Eq. 4.13, is always positive. The effective attraction arises due to the interaction of the instantons with the field $b_{1}(\tau)$, Eq. 4.6), and can be obtained calculating the first order $\epsilon_{s \mathbf{p}}^{(1)}, \mathrm{Eq}$. 4.8. Using Eq. 3.45 one can easily carry out summation over $n$ to obtain Eq. (4.6) with the constant $J$,

$$
J=\frac{1}{2} \int \frac{\operatorname{sgn}\left(\varepsilon^{-}(\mathbf{p})\right)}{\sqrt{\left(\left(\varepsilon^{-}(\mathbf{p})\right)^{2}+\gamma^{2} \frac{(1-k)^{2}}{4}\right)\left(\left(\varepsilon^{-}(\mathbf{p})\right)^{2}+\gamma^{2}\right)}} \frac{d \mathbf{p}}{(2 \pi)^{2}} .
$$

At small $1-k$ the integral $J$, Eq. 4.14, can be very large and the attraction very strong due to a large contribution coming from the region of small $\varepsilon^{-}(\mathbf{p})$. Therefore, the second order $\epsilon_{s \mathbf{p}}^{(2)}$, Eq. 4.9$)$, is also important because it leads to a screening of the interaction (4.6, 4.14) and partially cuts the singularity at $\varepsilon^{-}(\mathbf{p})=0$ arising in the integrand in (4.14) in the limit $k \rightarrow 1$.

As a result, one can write the difference $\Delta \mathcal{F}\left[b_{1}, \delta b\right]$ between the free energy of the system with $m$ alternating instantons and antiinstantons and that of the system without instantons as

$$
\Delta \mathcal{F}\left[b_{1}, \delta b\right]=F_{\text {inst }}+\mathcal{F}_{\text {int }}\left[b_{1}\right]+\mathcal{F}_{2}\left[b_{1}, \delta b\right]
$$

with $F_{\text {inst }}$ given by Eq. $4.13, \mathcal{F}_{\text {int }}\left[b_{1}\right]$ specified by Eqs. 4.6 4.14 and a quadratic form $\mathcal{F}_{2}\left[b_{1}, \delta b\right]$ of $b_{1}(\tau)$ and $\delta b(\tau)$

$$
\begin{aligned}
& \frac{\mathcal{F}_{2}\left[b_{1}, \delta b\right]}{V T} \\
= & \int_{0}^{1 / T}\left[\left(\mathcal{A}_{0}+\frac{1}{4}\left(\mathcal{A}_{1}-\frac{\mathcal{C}^{2}}{\mathcal{B}}\right) \dot{b}_{0}^{2}(\tau)\right) b_{1}^{2}(\tau)+\right. \\
& \left.+\mathcal{B} b_{0}^{2}(\tau)\left(\delta b(\tau)-\frac{\mathcal{C} b_{0}(\tau)}{2 \mathcal{B} b_{0}(\tau)} b_{1}(\tau)\right)^{2}\right] d \tau
\end{aligned}
$$

where the constants $\mathcal{A}_{0}, \mathcal{A}_{1}, \mathcal{B}$ and $\mathcal{C}$ equal

$$
\begin{gathered}
\mathcal{A}_{0}=\left(1+\frac{U_{0}}{\tilde{U}_{0}}\right) \int \frac{1}{\sqrt{\left(\varepsilon^{-}(\mathbf{p})\right)^{2}+\gamma^{2}}} \frac{d \mathbf{p}}{(2 \pi)^{2}}, \\
\mathcal{A}_{1}=\int \frac{1}{\left(\left(\varepsilon^{-}(\mathbf{p})\right)^{2}+\gamma^{2}\right)^{3 / 2}\left(\left(\varepsilon^{-}(\mathbf{p})\right)^{2}+\gamma^{2} \frac{(1-k)^{2}}{4}\right)} \frac{d \mathbf{p}}{(2 \pi)^{2}},
\end{gathered}
$$

$$
\begin{gathered}
\mathcal{B}=\frac{1}{\left(\left(\varepsilon^{-}(\mathbf{p})\right)^{2}+\gamma^{2}\right)^{3 / 2}} \frac{d \mathbf{p}}{(2 \pi)^{2}}, \\
\mathcal{C}=\int \frac{\varepsilon^{-}(\mathbf{p})}{\left(\left(\varepsilon^{-}(\mathbf{p})\right)^{2}+\gamma^{2}\right)^{3 / 2}\left(\left(\varepsilon^{-}(\mathbf{p})\right)^{2}+\frac{\gamma^{2}(1-k)^{2}}{4}\right)} \frac{d \mathbf{p}}{(2 \pi)^{2}} .
\end{gathered}
$$

The minimum of $\mathcal{F}_{2}\left[b_{1}, \delta b\right]$ with respect to $\delta b(\tau)$ is achieved at

$$
\delta b(\tau)=\frac{\mathcal{C} \dot{b}_{0}(\tau)}{2 \mathcal{B} b_{0}(\tau)} b_{1}(\tau) .
$$

Then, one finds the minimum value of $\Delta \mathcal{F}\left[b_{1}, \delta b\right]$, Eq. 4.15) giving the free energy $\Delta F$

$$
\begin{aligned}
& \frac{\Delta F}{V T}=\frac{F_{\text {inst }}}{V T} \\
& -J^{2} \int_{0}^{1 / T}\left[\mathcal{A}_{0}+\frac{1}{4}\left(\mathcal{A}_{1}-\frac{\mathcal{C}^{2}}{\mathcal{B}_{0}}\right) \dot{b}_{0}^{2}(\tau)\right]^{-1} \dot{b}_{0}^{2}(\tau) d \tau,
\end{aligned}
$$

where $\Delta F$ is the difference between the total free energy $F$ and the free energy $F_{\text {hom }}$ of the system with the order parameter homogeneous in the imaginary time.

At first glance, the energy $\Delta F$ is proportional to $T$, which is small at low temperatures. However, in the limit of small $k$, when the period of the oscillations is large, the energy $\Delta F$ is proportional to the number $m$, that can be large and proportional to $1 / T$. The energy $\Delta F$ is proportional to the volume as well, and therefore the contribution of the IAP into thermodynamical quantities will exceed those coming from fluctuations. The case $\Delta F /(2 m T V)>0$ corresponds to the state with the static order parameter, while in the region of parameters where $\Delta F / V(2 m T)<0$ one expects a chain of alternating instantons and anti-instantons. A more accurate calculations are necessary to determine the number $m$ as a function of temperature and parameters of the model. Here we restrict ourselves by investigating the stability against formation the chain of the alternating instanton and antiinstantons.

The main contribution to the integral, Eq. 4.22, comes from the vicinity of zeros of $b_{0}(\tau)$, where the derivative $\dot{b}_{0}(\tau)$ is essentially non-zero. Therefore, the integral is proportional to $2 m$ (as well as $F_{\text {inst }}$, Eq. 4.13), and the integration is reduced to the integration over the half period of the function $b_{0}(\tau)$. The free energy $\Delta F$ is also proportional to $2 m$, and one can calculate the energy per one instanton replacing $b_{0}(\tau)$ by $\gamma \tanh \gamma \tau$ and then integrating over $\tau$ from $-\infty$ to $\infty$ because the distance between the instantons and antiinstantons is very large in the limit $k \rightarrow 1$. Equation 4.22 can be further simplified introducing a new variable of integration $v=\gamma \tanh \gamma \tau$.

In order to compute the energy $\Delta F$ explicitly one should choose a specific form of the electron spectrum. 
Having in mind SFMOHS ${ }^{21}$ we write the spectrum as

$\varepsilon_{1}(\mathbf{p})=\alpha p_{x}^{2}-\beta p_{y}^{2}+P-\mu, \quad \varepsilon_{2}(\mathbf{p})=\alpha p_{y}^{2}-\beta p_{x}^{2}-P-\mu$,

where $\mu$ is the chemical potential and $P$ is a Pomeranchuk order parameter that may appear in the model under consideration. The spectrum displayed in Eq. 4.23 corresponds at $P=0$ to the Fermi surface depicted in Fig. 17. In principle, the Pomeranchuk order parameter can compete with the DDW and the state with the instantons and antiinstantons, and one should then consider all these phases together. In order to avoid too complicated formulas here, we neglect the inverse effect of the instantons of the value of $P$ and consider the latter as an independent parameter. We introduce also an energy cutoff $\Lambda$ limiting the areas of the hot spots as

$$
\frac{\alpha+\beta}{2} p^{2}<\Lambda
$$

with $p^{2}=p_{x}^{2}+p_{y}^{2}$.

The dependence of a function $S=\Delta F /(2 m T V)$ on parameters characterizing the energy spectrum in the SFMOHS is represented in Fig. 3. We use the following notations

$$
a=U_{0} / \tilde{U}_{0}, g_{0}=\frac{1}{2 \pi} \frac{U_{0}}{\alpha+\beta} .
$$

Figs. 3(a-e) represent dependence of $z=2 \pi^{2}(\alpha+\beta) S / \Lambda$ on $x=P / \gamma$ and $y=\Lambda / \gamma$ for several values of $k$ and $a$. Fig. 3: describes the solution of the self-consistency equation (3.17) for arbitrary $\Lambda$ and $P$. More information about the computations can be found in the Appendix.

We see from Figs. 3 that there is a certain region of parameters where the free energy $\Delta F$ is negative, which indicates the instability of the imaginary-time-independent state (DDW). The region of small $1-k$ and $a$ is most favorable for the formation of the lattice of IAP. As we consider here structures periodic in space (oscillations with vector $\mathbf{Q}_{A F}$ connecting the bands 1 and 2, Fig. 1 b), the periodic in $\tau$ order parameter $b(\tau)$ providing the minimum of the free energy is at the same time the amplitude of the periodic oscillations in space.

The present calculations do not determine the number $m$ of IAP as a functions of temperature. Leaving this problem for future investigations, we will calculate in the next sections physical quantities without specifying the value of $m$ or, alternatively, $k$ related to the latter by Eq. (3.20).

\section{THERMODYNAMIC QUANTUM TIME-SPACE CRYSTAL.}

In the previous sections we have shown that the state with the order parameter $b(\tau)$ represented by a chain of alternating instantons and antiinstantons in the imaginary time can be more favorable energetically than the one with the static order parameter. Now we discuss properties of this state concerning its behavior in real time.

The thermodynamical quantities have been calculated using imaginary time $\tau$ and Matsubara frequencies. Methods of calculations of dynamic quantities for "conventional systems' are well developed. Linear response functions are calculated by an analytical continuation from imaginary Matsubara frequencies to real ones ${ }^{95}$. This method is based on an assumption that the response function is analytical at $\infty$ on the complex plane of the frequencies. However, due to periodicity in both real $t$ and imaginary time $\tau$ of the function $b_{0}(\tau)$, Eq. (3.19), one obtains a Fourier series rather than a Fourier integral in the complex plane of the frequencies and the standard analytical continuation cannot be applied for this essentially thermodynamic problem.

Fortunately, one can use now the fact that the function $b_{0}(\tau)$, Eq. 3.19$)$, is analytical in the complex plane of $\tau$ (except poles). Equation (3.23) as well as equations 3.28 3.31 can be used everywhere on this plane including the axis of real time $t=-i \tau$.

At zero temperature $T=0$, one can rather easily represent real-time correlation functions in terms of a functional integral in real time. This is the standard fieldtheoretical formulation of quantum mechanics in terms of path integrals. Following this method one represents the current-current correlation function $N\left(t_{1}-t_{2}\right)$ in the form

$$
\begin{aligned}
& N\left(t_{1}-t_{2}\right) \\
= & \frac{U_{0}^{2}}{V^{2}} \sum_{\mathbf{p}, \mathbf{p}^{\prime}, \alpha, \alpha^{\prime}}\left\langle\left(\eta_{p}^{+}\left(t_{1}\right) \Sigma_{3} \eta_{p}\left(t_{1}\right)\right)\left(\eta_{p^{\prime}}^{+}\left(t_{2}\right) \Sigma_{3} \eta_{p^{\prime}}\left(t_{2}\right)\right)\right\rangle_{\tilde{S}} .
\end{aligned}
$$

In Eq. (5.1) the same fields $\eta, \eta^{+}$as in the previous sections are used. However, they are functions of real time, and the angle brackets stand for averaging with the action written in real time. The function $N\left(t_{1}-t_{2}\right)$ is proportional to a function entering the scattering crosssections. The overall coefficient in Eq. (5.1) is chosen for simplicity.

The equivalent functional integration is based on averaging with action $\tilde{S}$ written in real time

$$
\langle\ldots\rangle_{\tilde{S}}=\frac{\int(\ldots) e^{-i \tilde{S}[\eta]} D \eta}{\int e^{-i \tilde{S}[\eta]} D \eta} .
$$

In Eq. $[5.2$, the action $\tilde{S}[\eta]$ has the following form

$$
\tilde{S}_{0}[\eta]=\tilde{S}_{0}[\eta]+\tilde{S}_{\mathrm{int}}[\eta]
$$

where

$\tilde{S}_{0}[\eta]=\sum_{p} \int_{-\infty}^{\infty} \eta_{p}^{+}(t)\left(-i \partial_{t}+\varepsilon^{+}(\mathbf{p})-\varepsilon^{-}(\mathbf{p}) \Sigma_{2}\right) \eta_{p}(t) d t$, 


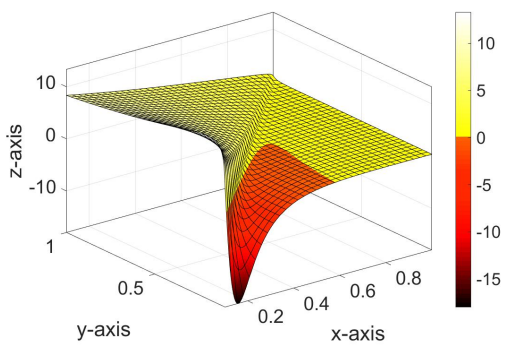

(a) $k=0.99, a=0$

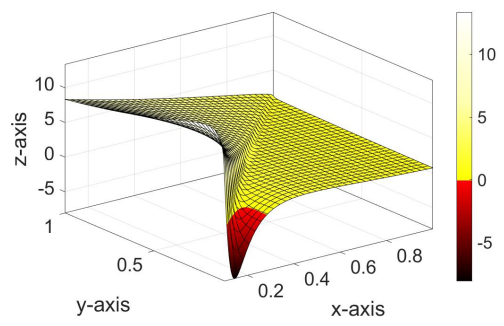

(d) $k=0.99, a=1$

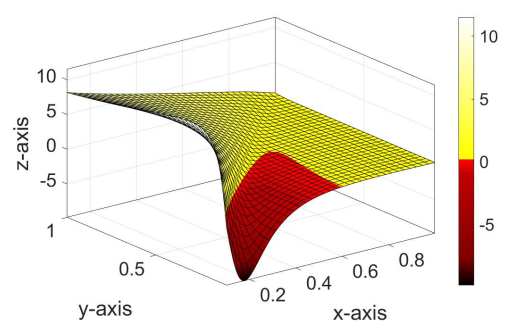

(b) $k=0.90, a=0$

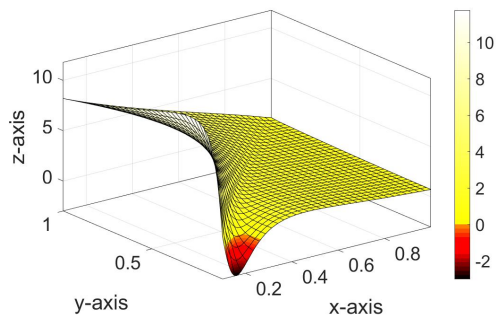

(e) $k=0.90, a=1$

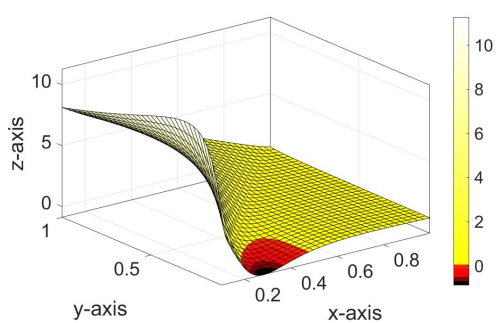

(c) $k=0.70, a=0$

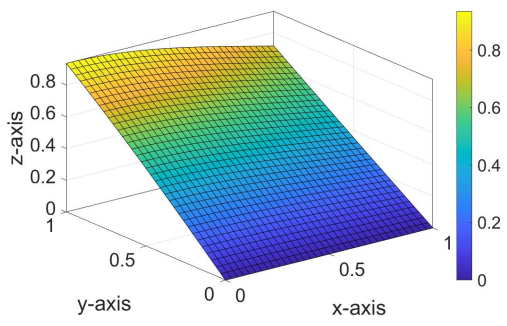

(f) $z=g_{0}^{-1}(x, y)$

FIG. 3: (Color online.) Free energy of instanton-antiinstanton pairs.

and

$$
\begin{aligned}
& \tilde{S}_{\text {int }}[\chi]=-\frac{1}{4 V} \int_{-\infty}^{\infty}\left[U_{0}\left(\sum_{p} \eta_{p}^{+}(t) \Sigma_{3} \eta_{p}(t)\right)^{2}\right. \\
& \left.-\tilde{U}_{0}\left(\sum_{p} \eta_{p}^{+}(t) \Sigma_{1} \eta_{p}(t)\right)^{2}\right] d t .
\end{aligned}
$$

The action $\tilde{S}[\eta]$ can easily be obtained from Eqs. 3.4 . 3.6 making the Wick rotation $\tau \rightarrow i t$. Again, the evaluation of the functional integrals can be performed decoupling the interaction by Gaussian integration over auxiliary fields $B(t)$ and $B_{1}(t)$. This leads us to the electron part of action $\mathcal{S}\left[\eta, \eta^{+}, B, B_{1}(t)\right]$ containing both fermion $\eta, \eta^{+}$and boson $B(t), B_{1}(t)$ fields

$$
\mathcal{S}\left[\eta \eta^{+}, B, B_{1}(t)\right]=\int_{-\infty}^{\infty} \eta_{p}^{+}(t) \mathcal{H}(t, \mathbf{p}) \eta_{p}(t) d t
$$

with the operator $\mathcal{H}(t, \mathbf{p})$ equal to

$$
\mathcal{H}(t, \mathbf{p})=-i \partial_{t}+\varepsilon^{+}(\mathbf{p})-\mathcal{H}_{0}(t, \mathbf{p}),
$$

where

$$
\mathcal{H}_{0}(t, \mathbf{p})=\varepsilon^{-}(\mathbf{p}) \Sigma_{2}+i\left(B(t) \Sigma_{3}+B_{1}(t) \Sigma_{1}\right) .
$$

Then, we integrate over $\eta, \eta^{+}$and reduce the full action $S$ to the form

$$
S=-\ln \left[\int \exp \left[-i \mathcal{S}\left[B, B_{1}\right]\right] D B D B_{1}\right],
$$

where the action $\mathcal{S}\left[B, B_{1}\right]$ equals

$$
\begin{aligned}
\mathcal{S}\left[B, B_{1}\right]= & \int_{-\infty-}^{\infty}\left[-2 \sum_{\mathbf{p}} \operatorname{tr}[\ln (\mathcal{H}(t, \mathbf{p}))]_{t, t}\right. \\
& \left.-V\left(\frac{B^{2}(t)}{U_{0}}-\frac{B_{1}^{2}(t)}{\tilde{U}_{0}}\right)\right] d t
\end{aligned}
$$

Minimizing $\mathcal{S}\left[B, B_{1}\right]$ with respect to $B(t)$ and $B_{1}(t)$ we come to equations

$$
\begin{aligned}
B(t) & =-i U_{0} \operatorname{tr} \int \Sigma_{3} \mathcal{G}_{\mathbf{p}}\left(t, t^{\prime}\right) \frac{d \mathbf{p}}{(2 \pi)^{2}}, \\
B_{1}(t) & =i \tilde{U}_{0} \operatorname{tr} \int \Sigma_{1} \mathcal{G}_{\mathbf{p}}\left(t, t^{\prime}\right) \frac{d \mathbf{p}}{(2 \pi)^{2}},
\end{aligned}
$$

where the matrix Green function $\mathcal{G}_{\mathbf{p}}\left(t, t^{\prime}\right)$ satisfies the equations

$$
\begin{aligned}
\mathcal{H}(t, \mathbf{p}) \mathcal{G}_{\mathbf{p}}\left(t, t^{\prime}\right) & =-\delta\left(t-t^{\prime}\right), \\
\mathcal{G}_{\mathbf{p}}\left(t, t^{\prime}\right) \overline{\mathcal{H}}\left(t^{\prime}, \mathbf{p}\right) & =-\delta\left(t-t^{\prime}\right) .
\end{aligned}
$$

In Eq. 5.17) the operator $\overline{\mathcal{H}}(t, \mathbf{p})$ equals

$$
\overline{\mathcal{H}}(t, \mathbf{p})=i \overleftarrow{\partial}_{t}+\varepsilon^{+}(\mathbf{p})-\mathcal{H}_{0}(t, \mathbf{p})
$$

We will see that the solutions $B(t), B_{1}(t)$ are periodic. The integrands in Eqs. 5.11, 5.12 can be calculated using spectral expansions similar to those performed in Sections (III, IV). Indeed, one can introduce eigenfunctions $\psi_{s \mathbf{p}}(t, \mathbf{p})$ and eigenvalues $E_{s \mathbf{p}}$ of the operator $\mathcal{H}(t, \mathbf{p})$, Eq. (5.7), as

$$
\begin{aligned}
& \mathcal{H}(t, \mathbf{p}) \psi_{s \mathbf{p}}(t)=E_{s \mathbf{p}} \psi_{s \mathbf{p}}(t), \\
& \bar{\psi}_{s \mathbf{p}}(t) \overline{\mathcal{H}}(t, \mathbf{p})=E_{s \mathbf{p}} \bar{\psi}_{s \mathbf{p}}(t) .
\end{aligned}
$$


Equations 5.11,5.12), and (5.16) are very similar to Eqs. 3.12 3.13 and (4.1), respectively, and can be obtained from those setting $T=0$ and making the rotation $\tau \rightarrow i t$.

It can rather easily be demonstrated that there are real periodic solutions $B(t)$ and $B_{1}(t)$ of Eqs. 5.11 5.12 determining the minimum of the action $\mathcal{S}\left[B, B_{1}\right]$, Eq. 5.10. Indeed, subtracting Eqs. 5.13, 5.14 from each other one obtains putting $t^{\prime}=t$ the following equation

$$
-i \partial_{t}\left(\mathcal{G}_{\mathbf{p}}(t, t)\right)-\left[\mathcal{H}_{0}(t, \mathbf{p}), \mathcal{G}_{\mathbf{p}}(t, t)\right]=0,
$$

where $[. ., .$.$] stands for the commutator.$

Representing the solution $\mathcal{G}_{\mathbf{p}}(t, t)$ in the form

$$
\mathcal{G}_{\mathbf{p}}(t, t)=i S_{\mathbf{p}}^{1}(t) \Sigma_{1}+S_{\mathbf{p}}^{2}(t) \Sigma_{2}+i S_{\mathbf{p}}^{3} \Sigma_{3},
$$

one can transform Eq. 5.18 as

$$
\partial_{t} \mathbf{S}_{\mathbf{p}}(t)=2 \mathbf{B}_{\mathbf{p}}(t) \times \mathbf{S}_{\mathbf{p}}(t)
$$

where the vectors $\mathbf{S}_{\mathbf{p}}(t)$ and $\mathbf{B}_{\mathbf{p}}(t)$ equal

$$
\begin{aligned}
& \mathbf{S}_{\mathbf{p}}(t)=\left(S_{\mathbf{p}}^{1}(t), S_{\mathbf{p}}^{2}(t), S_{\mathbf{p}}^{3}(t)\right), \\
& \mathbf{B}_{\mathbf{p}}(t)=\left(B_{1}(t), \varepsilon^{-}(\mathbf{p}), B(t)\right) .
\end{aligned}
$$

The vector product in Eq. 5.19) is defined for arbitrary 3-component vectors $\mathbf{L}$ and $\mathbf{M}$ as

$$
[\mathbf{L} \times \mathbf{M}]^{k}=e_{i j k} g_{k k} L^{i} L^{j}
$$

where $e_{i j k}$ is the antisymmetric tensor $\left(e_{123}=1\right)$, and $g=\operatorname{diag}(-1,1,-1)$ is metric. Accordingly, a scalar product of the vectors $\mathbf{L}$ and $\mathbf{M}$ equals

$$
(\mathbf{L M})=\sum_{k} g_{k k} L^{k} M^{k}=-L^{1} M^{1}+L^{2} M^{2}-L^{3} M^{3} .
$$

Using the definitions $5.21,5.22$ we multiply both the sides of Eq. 5.19 by the vector $\mathbf{S}_{\mathbf{p}}(t)$ to obtain

$$
\partial_{t} \mathbf{S}_{\mathbf{p}}^{2}(t)=0, \quad \mathbf{S}_{\mathbf{p}}^{2}(t)=q_{\mathbf{p}}^{2}
$$

In Eq. 5.23, $q_{\mathbf{p}}$ is a time-independent function of $\mathbf{p}$. Further, Eqs. 5.11, 5.12) can be rewritten in the form

$$
\begin{gathered}
B(t)=2 U_{0} \int S_{\mathbf{p}}^{3}(t) \frac{d \mathbf{p}}{(2 \pi)^{2}}, \\
B_{1}(t)=-2 \tilde{U}_{0} \int S_{\mathbf{p}}^{1}(t) \frac{d \mathbf{p}}{(2 \pi)^{2}} .
\end{gathered}
$$

Assuming that the functions $B(t)$ and $B_{1}(t)$ are periodic in time with a period $T_{0}$ one can see using Eqs. 5.19 5.23 that $\mathbf{S}_{\mathbf{p}}(t)$ is also periodic with the same period.

Indeed, one writes in this case the equation for $\mathbf{S}_{\mathbf{p}}\left(t+T_{0}\right)$ as

$$
\partial_{t} \mathbf{S}_{\mathbf{p}}\left(t+T_{0}\right)=2 \mathbf{S}_{\mathbf{p}}\left(t+T_{0}\right) \times \mathbf{B}_{\mathbf{p}}(t),
$$

and one can write the solution in the form

$$
\mathbf{S}_{\mathbf{p}}\left(t+T_{0}\right)=w_{\mathbf{p}} \mathbf{S}_{\mathbf{p}}(t),
$$

where $w_{\mathbf{p}}$ is a time-independent constant. Using Eq. $5.23,5.24,5.25$ one comes immediately to conclusion that $w_{\mathbf{p}}=1$, and the solution $\mathbf{S}_{\mathbf{p}}(t)$ is also periodic. The periodicity property can be written as

$$
\mathbf{B}\left(t+T_{0}\right)=\mathbf{B}(t), \quad \mathbf{S}_{\mathbf{p}}(t)=\mathbf{S}_{\mathbf{p}}\left(t+T_{0}\right) .
$$

So, one can expect periodic functions $B(t), B_{1}(t)$ providing the minimum of the action $\mathcal{S}\left[B, B_{1}\right]$, Eq. (5.10). Equations 5.19,5.25 allow one to fix symmetries of the solutions

$$
\begin{aligned}
B(t) & =-B(-t), \quad B_{1}(t)=B_{1}(-t), \\
S^{3}(t) & =-S^{3}(-t), \quad S^{1}(t)=S^{1}(-t), \quad S^{2}(t)=S^{2}(-t) .
\end{aligned}
$$

Comparing Eqs. 5.11, 5.12 with Eqs. 3.12, 3.13 derived when minimizing the free energy functional $\mathcal{F}\left[b, b_{1}\right]$, Eqs. 3.10, 3.11, we conclude that

$$
i B(t)=b(i t), \quad B_{1}(t)=b_{1}(i t) .
$$

It is very important that if $B(t)$ and $B_{1}(t)$ are solutions of Eqs. 5.11, 5.12), then $B\left(t-t_{0}\right)$ and $B_{1}\left(t-t_{0}\right)$ are also solutions at an arbitrary $t_{0}$. It is clear that there can be many solutions even at a fixed $t_{0}$. For example, for $B_{1}(t)=0$ one comes in the limit $k \rightarrow 1$ to Eq. (3.17) for any period of the function $B(t)$. The relation $(5.30)$ allows one to obtain proper $B(t)$ and $B_{1}(t)$ as soon as $b(\tau)$ and $b_{1}(\tau)$ are obtained from the condition for the minimum of the free energy functional $\mathcal{F}\left[b, b_{1}\right]$, Eq. (3.10).

Now, using Eq. (5.1, 5.7) we can integrate over the fermion fields $\eta, \eta^{+}$in Eq. (5.1) to obtain

$$
N\left(t_{1}-t_{2}\right)=-U_{0}^{2} \overline{\int \operatorname{tr}\left[\Sigma_{3} \mathcal{G}\left(t_{1}-t_{0}, \mathbf{p}_{1}\right)\right]_{t_{1} t_{1}} \frac{d \mathbf{p}_{1}}{(2 \pi)^{2}} \int \operatorname{tr}\left[\Sigma_{3} \mathcal{G}\left(t_{2}-t_{0}, \mathbf{p}_{2}\right)\right]_{t_{2} t_{2}} \frac{d \mathbf{p}_{2}}{(2 \pi)^{2}}}
$$


necessary because the extremum of the action functional is degenerate with the respect to the time shifts, and one should integrate over all the extremum states. Finally, using Eq. (5.11) we write the correlation function $N(t)$ in the form

$$
N\left(t_{1}-t_{2}\right)=\overline{B\left(t_{1}-t_{0}\right) B\left(t_{2}-t_{0}\right)},
$$

where the function $B(t)$ is the exact periodic solution of Eqs. (5.11,5.15) (or Eq. (5.19 5.25)).

The loop current around the elementary cell is proportional to

$$
i U_{0} \overline{\operatorname{tr}\left[\Sigma_{3} \mathcal{G}\left(t-t_{0}, \mathbf{p}_{1}\right)\right]}=\overline{B\left(t-t_{0}\right)} .
$$

The periodicity of the exact solution guarantees a nondecaying oscillating behavior of the function $N\left(t_{1}-t_{2}\right)$, Eq. 5.32). As it is difficult to find the exact solution of Eqs. (5.19 5.25), we use the approximate solution $B_{0}(t)$ writing it with the help of Eq. (5.30) as

$$
B_{0}(t)=-i b_{0}(i t) \text {, }
$$

where $b_{0}(\tau)$ is the Jacobi elliptic function introduced in Eq. 3.23 .

The Jacobi elliptic function $\operatorname{sn}(i u, k)$ of an imaginary argument $i u$ is related to an antisymmetric elliptic function sc $(u \mid k)$ with the period $2 K(k)$ as 96

$$
\operatorname{sn}(i u \mid k)=i \operatorname{sc}\left(u \mid k^{\prime}\right), k^{2}+k^{\prime 2}=1,
$$

and one can write the order parameter $B\left(t-t_{0}\right)$ in real time in the form

$$
B_{0}\left(t-t_{0}\right)=-i b_{0}\left(i\left(t-t_{0}\right)\right)=\gamma k \mathrm{sc}\left(\gamma\left(t-t_{0}\right) \mid k^{\prime}\right) .
$$

In Eq. 5.33, $t_{0}$ is arbitrary and integrating over the degeneracy of the extremum one obtains immediately

$$
\overline{B\left(t-t_{0}\right)}=0,
$$

Comparing Eq. 5.37 with Eq. 5.33 we conclude that the loop currents equal zero at any time $t$, which means that there is no radiation, and the energy is conserved. As concerns the correlation function $N\left(t_{1}-t_{2}\right)$, one should insert $B\left(t-t_{0}\right)$ from Eq. 5.36 into Eq. (5.32) and average over $t_{0}$.

The Fourier transform of the function sc $\left(u \mid k^{\prime}\right)$ is well known 96

$$
\begin{aligned}
& \operatorname{sc}\left(u \mid k^{\prime}\right)=\frac{\pi}{2 k K\left(k^{\prime}\right)} \tan \frac{\pi u}{2 K\left(k^{\prime}\right)} \\
& +\frac{2 \pi}{k K\left(k^{\prime}\right)} \sum_{n=1}^{\infty}(-1)^{n} \frac{\exp \left(-\frac{2 \pi n K(k)}{K\left(k^{\prime}\right)}\right)}{1+\exp \left(-\frac{2 \pi n K(k)}{K\left(k^{\prime}\right)}\right)} \sin \left(\frac{n \pi u}{K\left(k^{\prime}\right)}\right)
\end{aligned}
$$

where $K(k)$ is the elliptic integral of the first kind.

The oscillating behavior of this function can, again, be understood from Fig. 2 a. Indeed, the oscillation in the imaginary time $\tau$ could be visualized as classical motion of a particle in the potential $w=\frac{1}{2}\left(u^{2}\left(1+k^{2}\right)-u^{4}\right)$.
Therefore, the motion in real time should be described by motion in the potential $\tilde{w}(u)=-w(u)$ and is periodic.

Using the Fourier series for $\tan u$

$$
\tan u=-2 \sum_{n=1}^{\infty}(-1)^{n} \sin (2 n u),
$$

we write the function sc $\left(u \mid k^{\prime}\right)$ in a more compact form

$\operatorname{sc}\left(u \mid k^{\prime}\right)=-\frac{\pi}{k K\left(k^{\prime}\right)} \sum_{n=1}^{\infty}(-1)^{n} \tanh \frac{\pi n K(k)}{K\left(k^{\prime}\right)} \sin \frac{n \pi u}{K\left(k^{\prime}\right)}$.

Writing in Eq. (5.39) $u=\gamma\left(t-t_{0}\right)$ and substituting it into Eq. 5.36, 5.32) we write the correlation function $N(t)$ as

$$
N(t)=2 \gamma^{2} \sum_{n=1}^{\infty} f_{n}^{2} \cos \left(n \omega_{0} t\right)
$$

where

$$
f_{n}=\frac{\pi}{2 K\left(k^{\prime}\right)} \tanh \frac{\pi n K(k)}{K\left(k^{\prime}\right)}, \omega_{0}=\frac{\pi \gamma}{K\left(k^{\prime}\right)} .
$$

In the limit $k \rightarrow 1$ one has the following asymptotic behavior for the elliptic integral

$$
K(k) \propto \frac{1}{2} \ln \frac{8}{1-k}, \quad K\left(k^{\prime}\right) \propto \frac{\pi}{2},
$$

and Eq. 5.40, 5.41 simplifies to the following form

$$
N(t) \approx 2 \gamma^{2} \sum_{n=1}^{\infty}\left[1-\left(\frac{1-k}{8}\right)^{2 n}\right] \cos (2 \gamma n t) .
$$

In the limit $k \rightarrow 1$, the correlation function $N(t)$ shows an oscillating behavior with the frequencies $2 \gamma n$ (we put everywhere $\hbar=1$ ). The energy $2 \gamma$ is the energy of the breaking of electron-hole pairs and one can interpret the result $(5.43)$ as oscillations between the static order and normal state. The oscillating form of $N\left(t_{1}-t_{2}\right)$ resembles oscillations of the order parameter in the non-equilibrium superconductors $97-103]$ but, in contrast to those, the function $N(t)$ does not decay in time. Non-perturbative quantum dynamic effects have been studied in Ref. ${ }^{104}$ using the imaginary time representation. One obtains the non-decaying behavior because now a thermodynamically stable state is considered. The contribution of high harmonics $n$ does not decay with $n$, which originates from the existence of the poles in the first term in Eq. (5.38). Apparently, this is a consequence of using the function $B_{0}(t)$, Eq. (5.34), instead of the exact solution $B(t)$ in Eq. 5.32 , and more accurate calculations would give decaying amplitudes of high harmonics.

At the same time, the non-decaying form the function $N(t)$ is guaranteed by the periodicity of the solution $B(t)$, Eqs. (5.28), and the corresponding possibility of expanding this solution in Fourier series like those written in Eq. 5.38. 


\section{OPERATOR ORDER PARAMETER.}

The correlation function $N\left(t_{1}-t_{2}\right)$ of the functions $B(t)$ was calculated by averaging the product $B\left(t_{1}-t_{0}\right) B\left(t_{2}-t_{0}\right)$ over the position $t_{0}$. The same results for the correlation functions can be obtained using an alternative description based on the notion of an 'operator order parameter' $\hat{B}$. In order to describe the oscillating behavior of the time crystal one can formally introduce a Hamiltonian $\hat{H}_{T C}$ of a harmonic oscillator

$$
\hat{H}_{T C}=\left(a^{+} a+\frac{1}{2}\right) \omega_{0},
$$

where the energy $\omega_{0}$ has been introduced in Eqs. 5.40 , 5.41 , and $a^{+}, a$ are boson creation and annihilation operators satisfying the commutation relations

$$
a a^{+}-a a^{+}=1 \text {. }
$$

Instead of averaging over $t_{0}$ we represent now correlation functions a form of quantum mechanical averages with the Hamiltonian $\hat{H}_{T C}$. For this purpose we write for any $t$ and $t_{0}$ the following identity

$$
\exp \left(i n \omega_{0}\left(t+t_{0}\right)\right)|n\rangle=\frac{\left(e^{i \omega_{0}\left(t+t_{0}\right)} a^{+}\right)^{n}}{\sqrt{n !}}|0\rangle
$$

where $|n\rangle$ means $n$-th state of the Hamiltonian $\hat{H}_{T C}$, Eq. 6.1 .

Further, we have a standard relation

$$
a^{+}(t)=e^{i \hat{H}_{T C} t} a e^{-i \hat{H}_{T C} t}=a e^{i \omega_{0} t},
$$

which allows us to write

$$
\begin{aligned}
& e^{i n \omega_{0}\left(t+t_{0}\right)}|n\rangle \\
= & \frac{1}{\sqrt{n !}} e^{i \hat{H}_{T C}\left(t+t_{0}\right)}\left(a^{+}\right)^{n} e^{-i \hat{H}_{T C}\left(t+t_{0}\right)}|0\rangle .
\end{aligned}
$$

Introducing operator $A$

$$
A=\sum_{n=1}^{\infty} f_{n} \frac{a^{n}}{\sqrt{n !}}, A(t)=e^{i \hat{H}_{T C} t} A e^{-i \hat{H}_{T C} t},
$$

where $f_{n}$ is given by Eq. (5.41), and its Hermitian conjugate $A^{+}$, one obtains easily

$$
\begin{aligned}
A(t)|0\rangle & =0 \\
A^{+}(t)|0\rangle & =\sum_{n=1}^{\infty} f_{n} \frac{\left(a^{+}(t)\right)^{n}}{\sqrt{n !}}|0\rangle=\sum_{n=1}^{\infty} f_{n} e^{i n \omega_{0} t}|n\rangle .
\end{aligned}
$$

Now we consider two different methods of calculation of correlation functions. Following the first method we calculate the correlation functions by averaging over $t_{0}$,

$$
\sum_{n=1}^{\infty} f_{n} \overline{e^{i n \omega_{0}\left(t+t_{0}\right)}}=0
$$

$$
\sum_{n_{1}, n_{2}=1}^{\infty} f_{n_{1}} f_{n_{2}} \overline{e^{i n_{1} \omega_{0}\left(t+t_{0}\right)} e^{i n_{2} \omega_{0} t_{0}}}=0
$$

and

$$
\sum_{n_{1}, n_{2}=1}^{\infty} f_{n_{1}} f_{n_{2}} \overline{e^{i n_{1} \omega_{0}\left(t+t_{0}\right)} e^{-i \omega_{0} n_{2} t_{0}}}=\sum_{n=1}^{\infty} f_{n}^{2} \exp \left(i \omega_{0} n t\right)
$$

where the bar means averaging over $t_{0}$.

On the other hand, we can write using Eqs. (6.7) and the normalization of the states $|n\rangle$ the following relations

$$
\begin{gathered}
\sum_{n=1}^{\infty} f_{n} \overline{e^{i n \omega_{0}\left(t+t_{0}\right)}}=\left\langle 0\left|A^{+}\left(t+t_{0}\right)\right| 0\right\rangle=0 \\
\sum_{n_{1}, n_{2}=1}^{\infty} f_{n_{1}} f_{n_{2}} \overline{e^{i n_{1} \omega_{0}\left(t+t_{0}\right)} e^{-i n_{2} \omega_{0} t_{0}}}=\left\langle 0\left|A(0) A^{+}(t)\right| 0\right\rangle,
\end{gathered}
$$

and

$$
\sum_{n_{1}, n_{2}=1}^{\infty} f_{n_{1}} f_{n_{2}} \overline{e^{-i n_{1} \omega_{0}\left(t+t_{0}\right)} e^{i n_{2} \omega_{0} t_{0}}}=\left\langle 0\left|A(t) A^{+}(0)\right| 0\right\rangle .
$$

In the language of the quantized order parameter $A$, one can replace with the help of Eqs. 6.11 6.13 the averaging over the phase by a quantum mechanical averaging and write

$$
N(t)=\gamma^{2}\left(\left\langle 0\left|A(t) A^{+}(0)\right| 0\right\rangle+\left\langle 0\left|A(0) A^{+}(t)\right| 0\right\rangle\right) .
$$

where $|0\rangle$ stands for the wave function of the ground state of the Hamiltonian $\hat{H}_{T C}$, Eq. 6.1. At the same time, quantum averages of the operators $A$ and $A^{+}$vanish

$$
\langle 0|A(t)| 0\rangle=\left\langle 0\left|A^{+}(t)\right| 0\right\rangle=0 .
$$

Actually, using this representation one can calculate multi-time correlation functions

$$
=\frac{N_{2 p}\left(t_{1}, t_{2} \ldots, t_{2 p}\right)}{B\left(t_{1}\right) B\left(t_{2}\right) \ldots B\left(t_{p}\right) B\left(t_{p+1}\right) B\left(t_{p+2}\right) \ldots B\left(t_{2 p}\right)},
$$

with $B(t)$ determined by Eq. Writing $\sin \left(n \pi u / K\left(k^{\prime}\right)\right)$ in Eq. 5.39 as the sum of two exponentials one can multiply all $B\left(t_{l}\right)$ in Eq. (6.16) and average over $t_{0}$ term by term. It can be checked that each average can be written as a quantum-mechanical average of products of operators $A(t)$ and $A^{+}(t)$.

As a result, the correlation function $N_{2 p}\left(t_{1}, t_{2} \ldots, t_{2 p}\right)$ takes the form

$$
\begin{aligned}
& N_{2 p}\left(t_{1}, t_{2} \ldots, t_{2 p}\right) \\
= & \frac{1}{(p !)^{2}} \sum_{P}\left\langle 0\left|\prod_{0 \leq l \leq p} A\left(t_{l}\right) A^{+}\left(t_{p+l}\right)\right| 0\right\rangle .
\end{aligned}
$$

where, the symbol $\sum_{P}$ means the sum of all permutations of the operators $A\left(t_{l}\right)$ and $A^{+}\left(t_{l}\right)$ in the product. 
Correlation functions of odd number of times are equal to zero. At $p=1$ one obtains the correlation function $N\left(t_{1}-t_{2}\right)$, Eq. 6.14). In the limit $k \rightarrow 1$, Eq. 6.14 simplifies to $(5.43)$.

Equations (5.32, 6.14) and (6.16, 6.17) demonstrate equivalence between the averaging of classical order parameters over the positions in time and the quantummechanical averaging of operator order parameters. This resembles the equivalence between the coherent states and the number states descriptions in quantum optics $107 \mid 109$.

One can interpret the operator $A$ as an operator order parameter. This type of the order parameters extends the variety of conventional order parameters like scalars, vectors, matrices used in theoretical physics. As the quantum mechanical average of the operators $A$ and $A^{+}$vanishes, one cannot expect any loss of energy due to e.g. emission of light. At the same time, already twotime correlation functions do show oscillating behavior and this does not mean any loss of energy. The system described by the Hamiltonian $\hat{H}_{T C}$, Eq. 6.1), remains in the ground state, and the oscillations are due to virtual transitions between the states. Remarkably, the distance between the energy levels does not decay in the limit of infinite volume, $V \rightarrow \infty$, which demonstrates a coherence all over the sample.

The two-times correlation functions of type 5.436 .14 describe inelastic quantum mechanical scattering and corresponding experiments can be used for observing the time crystals. The non-decaying time oscillations can be an important property for designing qubits but, for making devices, one should identify physically relevant systems described by Eqs. 2.13 2.15.

\section{SPONTANEOUS BREAKING OF THE TIME-TRANSLATION SYMMETRY: TIME-DEPENDENT WAVE FUNCTIONS OF EQUILIBRIUM STATES AND THEIR MEANING.}

The oscillating behavior of the correlation function $N(t)$, Eqs. 5.40 5.43, has been demonstrated in the preceding sections using methods of functional integration. Within this method one starts writing physical quantities in a form of a functional integral over anticommuting fermion fields. These integrals are transformed to functional integrals over boson fields, and one can calculate these integrals using the saddle-point method. The oscillating in both imaginary and real time order parameters appears as a result of minimizing an effective free energy (action) functional of boson fields. As a result, correlation functions oscillating in real time arise in the thermodynamically stable state. This is the oscillating in time order parameter $B(t)$ that is responsible for this striking effect. Using a time-independent order parameter in Eqs. 5.31, 5.32 one would obtain a standard time-independent long-range order in space only.

However, previous discussions in the literature on the time-crystals were based on the more traditional Hamiltonian formalism (see, e.g. Ref!10). Assuming that the starting Hamiltonian does not depend on time it is less straightforward to understand an oscillating behavior of correlation functions at large times. Therefore, we sketch in this section the derivation of the main results within the Hamiltonian approach and clarify why the results do not agree with the conclusions of Ref 10 .

\section{A. Hamiltonian approach.}

We start this subsection with writing a Hamiltonian $\hat{H}$ corresponding to the action $S\left[\chi, \chi^{+}\right], \mathrm{Eq}$. 2.13. It describes the system of interacting electrons in two bands 1 and 2

$$
\begin{aligned}
\hat{H}= & \sum_{p} c_{p}^{+}\left(\varepsilon^{+}(\mathbf{p})+\varepsilon^{-}(\mathbf{p}) \Sigma_{3}\right) c_{p} \\
& +\frac{1}{4 V}\left[\tilde{U}_{0}\left(\sum_{p} c_{p}^{+} \Sigma_{1} c_{p}\right)^{2}-U_{0}\left(\sum_{p} c_{p}^{+} \Sigma_{2} c_{p}\right)^{2}\right] .
\end{aligned}
$$

Two-component vectors $c_{p}=\left\{c_{p}^{1}, c_{p}^{2}\right\}$, contain creation and destruction operators $c_{p}^{1}$ and $c_{p}^{2}$ for the fermions of the bands 1 and $2, p=\{\alpha, \mathbf{p}\}$, where $\alpha$ stands for spin (the spin variable $\alpha$ is not very important here). Hamiltonian (7.1) corresponds to the action $S[\chi]$, Eqs. 22.13. 2.15 , and the rest of the notations is the same. It resembles the BCS ${ }^{88}$ Hamiltonian in theory of superconductivity specially designed for describing superconductivity. The electron-electron interaction in Eq. 7.1 is short-ranged but the interaction terms have a somewhat simplified separable form containing summation over two momenta only. Equation (7.1) describes a Hamiltonian of the grand canonical ensemble written at fixed chemical potential $\mu$ (see also Eq. (2.5)). Considering grand canonical ensemble is standard in study of macroscopic systems of interacting electrons. Solving the model with the Hamiltonian $\hat{H}$, Eq. 7.1, is equivalent to solving models with a more general electron-electron interaction in the mean field approximation. In the limit of a large volume $V \rightarrow \infty$, one can replace the sum over the momenta by integrals using the standard replacement

$$
\sum_{p}(\ldots) \rightarrow V \int(\ldots) \frac{d \mathbf{p}}{(2 \pi)^{d}}
$$

( $d$ is dimension) and see that $\hat{H}$ is proportional to the volume $V$, as it should be. The form of the interaction in Eq. 7.1 corresponds the 'infinite range' interaction of effective electron-hole pairs (in the BCS Hamiltonian one writes interaction of electron-electron pairs), and that is why the mean field theory coincides with the exact solution for the Hamiltonian $\hat{H}$, Eq. 7.1). It is well known that, generally, the mean field approximation is not necessarily good in one and two dimensions but it works very well in three or quasi-two dimensional models at least qualitatively. Therefore, the Hamiltonian $\hat{H}$ is 
a good starting point for studying ordered phases of the electron systems in $3 \mathrm{D}$ or quasi-2D unless they are close to a phase transition.

Of course, time is not present in Eq. (7.1). It is very important for the results obtained in the present work that the coupling constant $\tilde{U}_{0}$ is positive and sufficiently large. In a recent publication ${ }^{110}$, similar models were considered with $\tilde{U}_{0}=0$ and $\tilde{U}_{0}=-U_{0}$, and it was demonstrated that the instanton lattice had a higher energy than the homogeneous state. The same result would be obtained if we used negative or not sufficiently large positive $\tilde{U}_{0}$.

Having in mind that the mean field approximation is exact for the model described by the Hamiltonian $\hat{H}$ we use this method for explicit calculations. One starts replacing $\hat{H}$, Eq. 7.1), by a mean field Hamiltonian containing an effective potential instead of the interparticle interaction. It can depend on the imaginary time $\tau$ when thermodynamic properties are studied or one can introduce a real-time dependence for studying real-time correlation functions. Introducing a mean field Hamiltonian is equivalent to decoupling the interaction by integration over auxiliary fields as it has been done in the previous sections. The effective free energy functional obtained in this way depends on the auxiliary field and, minimizing this functional, one obtains equations that are equivalent to the mean field equations. Of course, there can be many solutions of the mean field equations for the effective potential, and one should use the one corresponding to the minimum of the free energy. The difference between the free energies of different states is proportional to the volume $V$, and in the limit $V \rightarrow \infty$ one should find the one with the lowest energy.

\section{B. Time-dependent wave functions.}

Usually, one uses in quantum mechanics wave functions $\tilde{\Psi}(t, X)$ ( $X$ are coordinates of particles) that are solutions of the Schrödinger equation

$$
i \frac{\partial \tilde{\Psi}(t, X)}{\partial t}=\hat{H} \tilde{\Psi}(t, X) .
$$

The time-dependent solutions $\tilde{\Psi}(t, X)$ can be written in a form of a superposition

$$
\tilde{\Psi}(t, X)=\sum_{n} c_{n} \tilde{\Psi}_{n}(t, X),
$$

where the time-dependent eigenfunctions $\tilde{\Psi}_{n}(t, X)$ equal

$$
\tilde{\Psi}_{n}(t, X)=\Psi_{n}(X) \exp \left(-i E_{n} t\right),
$$

and the time-independent wave functions $\bar{\Psi}_{n}(X)$ satisfy the stationary Schrödinger equation

$$
\hat{H} \Psi_{n}(X)=E_{n} \Psi_{n}(X) .
$$

The time-dependent wave functions $\tilde{\Psi}_{n}(t, X)$ obey orthogonality relations

$$
\begin{aligned}
& \int \tilde{\Psi}_{n}^{*}(t, X) \tilde{\Psi}_{m}(t, X) d X \\
= & \int \exp \left(i\left(E_{n}-E_{m}\right) t\right) \Psi_{n}^{*}(X) \Psi_{m}(X) d X=\delta_{m n} .
\end{aligned}
$$

Solutions of the Schrödinger equation 7.3 are generally time-dependent but can be represented in a form of Eq. 7.4 7.5 with the help of the time-independent eigenfunctions $\Psi_{n}(X)$. The scalar product of two timedependent wave functions in Eq. (7.7) contains integration over the coordinates $X$ but not over time $t$.

As a spontaneous breaking of the symmetry is expected, the symmetries of the Hamiltonian may differ below the transition point from those of the original Hamiltonian $\hat{H}$. There are plenty of examples of such a behavior. One of the examples, most close to the present case, is formation of a charge density wave (CDW) in a model invariant with respect to space translations. Below the transition, a periodic in space order parameter appears, which leads to an additional periodic dependence of electron wave functions on coordinates (Bloch theorem). Thermodynamic quantities depend directly on the amplitude of CDW but not on the modulation vector $\mathbf{Q}$, although the latter enters explicitly the mean field Hamiltonian. At the same time, the space modulation can be observed in two-point correlation functions. One cannot calculate physical quantities like, e.g., correlation functions at different space points analytically without calculating first the order parameter. Mean field theory serves usually as an efficient tool for studying how the original symmetries of the model are broken. Fluctuations can in some cases be important in low dimensions but in 3D or in quasi-2D this procedure is in most cases reliable. One can say that the CDW appears as a result of the breaking of the space-translation symmetry of the original space-translation invariant Hamiltonian.

The scenario concerning formation of the thermodynamic quantum time crystal considered in this paper is similar. The main difference with respect to the formation of the CDW is the breaking of the timetranslation symmetry of the time-translation invariant (static) Hamiltonian. Being at the moment more interested in the correlation functions of real time, we will replace the Hamiltonian $\hat{H}$, Eq. (7.1), by a meanfield Hamiltonian $\hat{H}^{\mathrm{mf}}(t)$ quadratic in the operators $c_{p}$, $c_{p}^{+}$, which simplifies the calculations. The Hamiltonian $\hat{H}^{\mathrm{mf}}(t)$ contains periodic in time $t$ order parameters $B(t)$ and $B_{1}(t)$ that have to be found from mean-field equations. It is clear that the wave functions of $H^{\mathrm{mf}}(t)$ will depend on time in a non-trivial way different from Eqs. 7.5. 7.6. At first glance, one might think that such a mean field is simply unreasonable because it violates the exact form of the wave functions written in Eqs. 7.5 . 7.6).

In order to clarify this discrepancy we adopt now the scheme of the BCS theory ${ }^{88 \mid 111}$ to the present situation. 
Although we have to find solutions of the Schrödinger equation with the Hamiltonian $\hat{H}$, Eq. (7.1), written for the grand canonical ensemble, it is instructive to start with a system of a fixed number of electrons.

A trial antisymmerized time-dependent wave function $\tilde{\Psi}_{N}(t)$ of $N$ electrons and $N$ holes (spin indices are omitted for simplicity) can be written as

$$
\tilde{\Psi}_{N}(t)=A e^{-i E_{N} t} \prod_{j=1}^{N} \psi\left(\mathbf{r}_{e j}-\mathbf{r}_{h j}\right)
$$

where $\mathbf{r}_{e j}$ and $\mathbf{r}_{h j}, j=1,2,,,, N$ numerate coordinates of electrons and holes. Functions $\psi\left(\mathbf{r}_{e j}-\mathbf{r}_{h j}\right)$ satisfy a stationary Schrödinger equation for a single electronhole pair, the operator $A$ antisymmetrizes the product of these functions, and the energy $E_{N}$ is the total energy of $N$ isolated pairs. The Hamiltonian of a single electronhole pair is taken in the form

$$
\hat{h}_{\mathrm{eh}}\left(\mathbf{r}_{1}, \mathbf{r}_{2}\right)=\varepsilon_{e}\left(-i \nabla_{\mathbf{r}_{1}}\right)+\varepsilon_{h}\left(-i \nabla_{\mathbf{r}_{2}}\right)+V\left(\mathbf{r}_{1}-\mathbf{r}_{2}\right),
$$

where $\varepsilon_{e}\left(-i \nabla_{\mathbf{r}}\right)$ and $\varepsilon_{h}\left(-i \nabla_{\mathbf{r}}\right)$ are operators of kinetic energy of the electron and hole, and $V\left(\mathbf{r}_{1}-\mathbf{r}_{2}\right)$ is an interaction. Putting the pair in the center of mass we find the eigenvalue $\varepsilon_{0}$ and the eigenfunction $\psi\left(\mathbf{r}_{1}-\mathbf{r}_{2}\right)$ from the equation

$$
\hat{h}_{\mathrm{eh}}\left(\mathbf{r}_{1}, \mathbf{r}_{2}\right) \psi\left(\mathbf{r}_{1}-\mathbf{r}_{2}\right)=\varepsilon_{0} \psi\left(\mathbf{r}_{1}-\mathbf{r}_{2}\right) .
$$

It is clear that the energy $E_{N}$ of non-interacting electronhole pairs equals

$$
E_{N}=N \varepsilon_{0} .
$$

In principle, taking into account an effective interaction with other electron-hole pairs can change the value $\varepsilon_{0}$. In this case, one should use an energy $\varepsilon_{0 N}$ depending on $N$ instead of $\varepsilon_{0}$ and write

$$
E_{N}=N \varepsilon_{0 N}
$$

Introducing Fourier-transform of the function $\psi(\mathbf{r})$

$$
\psi(\mathbf{r})=\int g_{\mathbf{p}} e^{i \mathbf{p} r} \frac{d \mathbf{p}}{(2 \pi)^{d}},
$$

we write the function $\tilde{\Psi}_{N}(t)$ in the form

$$
\begin{aligned}
\tilde{\Psi}_{N}(t) & =A \prod_{j=1}^{N}\left(\sum_{\mathbf{p}_{j}} g_{\mathbf{p}_{j}} e^{i \mathbf{p}_{j}\left(\mathbf{r}_{e j}-\mathbf{r}_{h j}\right)} e^{-i \varepsilon_{0 N} t}\right) \\
& =\prod_{j=1}^{N}\left(\sum_{\mathbf{p}_{j}} g_{\mathbf{p}_{j}} c_{1 \mathbf{p}_{j}}^{+} c_{2 \mathbf{p}_{j}} e^{-i \varepsilon_{0 N} t}\right)|0\rangle .
\end{aligned}
$$

In Eq. 7.14 the symbol $|0\rangle$ stands for the ground state, and $c_{1 \mathbf{p}_{j}}, c_{2 \mathbf{p}_{j}}$ and $c_{1 \mathbf{p}_{j}}^{+}, c_{2 \mathbf{p}_{j}}^{+}$are destruction and creation operators for the state with momentum $\mathbf{p}$ of the $j$-th pair. All the transformations, Eqs. 77.87.14 have been exact so far, and the time dependence of the function $\tilde{\Psi}_{N}(t)$ has the form given by Eqs. 7.5 7.6 usual for static Hamiltonians.

The trial function $\tilde{\Psi}_{N}(t)$ is still too complicated and is not helpful for solving the Schrödinger equation with the Hamiltonian $\hat{H}$, Eq. 7.1). Moreover, it is written for the fixed number of electrons-hole pairs $N$, while the Hamiltonian $\hat{H}$ has been introduced for the grand canonical ensemble. Therefore, let us write a trial function $\tilde{\Phi}$ for the grand canonical ensemble in the form of a superposition

$$
\tilde{\Phi}(t)=\sum_{N=1}^{\infty} \lambda_{N} \tilde{\Psi}_{N}(t) .
$$

The coefficients $\lambda_{N}$ have to be chosen in a form that would make it possible to find exact time-dependent solutions for the Hamiltonian $\hat{H}$, Eq. 77.1). This task is achieved writing the function $\tilde{\Phi}(t)$ as

$$
\tilde{\Phi}(t)=\prod_{p}\left(u_{\mathbf{p}}(t)+v_{\mathbf{p}}(t) c_{p}^{1+} c_{p}^{2}\right)|0\rangle
$$

Equation (7.16) is written for $\varepsilon_{1}(\mathbf{p})>\varepsilon_{2}(\mathbf{p})$ (at $\varepsilon_{1}(\mathbf{p})<\varepsilon\left(\mathbf{p}_{2}\right)$ one should exchange the bands, $\left.1 \rightleftarrows 2\right)$, and $|0\rangle$ is the state of the Hamiltonian of non-interacting particles (Fermi step function). Equation (7.16) is very similar to the trial function in the BCS theory ${ }^{88}$ but the functions $u_{\mathbf{p}}(t), v_{\mathbf{p}}(t)$ depend on time, and the products $c_{p}^{1+} c_{p}^{2}$ create electron-hole pairs instead of the Cooper pairs. The superconducting analog of the function $\tilde{\Phi}(t)$, Eq. $(7.16)$, has been used in Ref ${ }^{[98}$ for studying nonequilibrium states in superconductors. The dependence of $\lambda_{N}$ on $N$ is sharp and $\left\langle N^{2}\right\rangle-\langle N\rangle^{2} \sim\langle N\rangle$, where $\langle N\rangle$ is the average number of electrons at a fixed chemical potential. At the same time, $\langle N\rangle \gg 1$, and the variation of $\lambda_{N}$ is negligible when several electron-hole pairs enter or leave the sample.

We emphasize that the function $\tilde{\Phi}(t)$, Eq. 7.16, is the most general form of the exact solution of the nonstationary Schrödinger equation with the Hamiltonian $\hat{H}$, Eq. (7.1), and this form is different from Eqs. (7.5, 7.6). Of course, Eq. (7.16) could be introduced as a guess without the preliminary discussion resulting in Eqs. 7.8 7.15). In this case, one would have to just check that the function $\tilde{\Phi}(t)$ is the solution to the Hamiltonian $\hat{H}$. However, this qualitative discussion may be helpful for understanding the origin of the form of the wave functions $\tilde{\Phi}(t)$, Eq. 7.16). Taking time-independent coefficients $u_{\mathbf{p}}$ and $v_{\mathbf{p}}$ in the trial function $\tilde{\Phi}$, Eq. (7.16), corresponds to the standard mean-field approximation used in Ref ${ }^{23}$.

Following the proposed mean field procedure we replace the Hamiltonian $\hat{H}$, Eq. 7.1), by the mean field Hamiltonian $\hat{H}^{\mathrm{mf}}(t)$, 


$$
\begin{aligned}
\hat{H} \rightarrow & \hat{H}^{\mathrm{mf}}(t)=\sum_{p} c_{p}^{+} M_{\mathbf{p}}^{\mathrm{mf}}(t) c_{p} \\
& -V\left(\frac{B^{2}(t)}{U_{0}}-\frac{B_{1}^{2}(t)}{\tilde{U}_{0}}\right)
\end{aligned}
$$

where

$$
\hat{M}_{\mathbf{p}}^{\mathrm{mf}}(t)=\varepsilon^{+}(\mathbf{p})+\varepsilon^{-}(\mathbf{p}) \Sigma_{3}-i\left(B(t) \Sigma_{2}+B_{1}(t) \Sigma_{1}\right),
$$

and functions $B(t)$ and $B_{1}(t)$ play the role of the order parameter. They are periodic in time with a period $T_{0}$ (these are the same functions as those introduced in Eqs. (5.6 5.12). Their explicit form of the time dependence can be obtained from self-consistency equations

$$
\begin{aligned}
& \frac{U_{0}}{2 V} \sum_{p}\left\langle c_{p}^{+} \Sigma_{2} c_{p}\right\rangle_{\mathrm{mf}}=B(t), \\
& \frac{\tilde{U}_{0}}{2 V} \sum_{p}\left\langle c_{p}^{+} \Sigma_{1} c_{p}\right\rangle_{\mathrm{mf}}=-B_{1}(t) .
\end{aligned}
$$

In Eqs. 7.197 .20 , the angle brackets $\langle\ldots\rangle_{\mathrm{mf}}$ stand for the quantum mechanical averaging with the Hamiltonian $\hat{H}^{\mathrm{mf}}(t)$, Eq. 7.17). Equations 7.19 7.20 are identical to Eqs. (5.11 5.12).

In order to find wave functions $\Phi$, one should solve the effective Schrödinger equation

$$
i \frac{\partial \tilde{\Phi}(t)}{\partial t}=H^{\mathrm{mf}}(t) \tilde{\Phi}(t) .
$$

The solutions $\tilde{\Phi}(t)$ of Eq. 7.21 are time-dependent but the expansion, Eqs. 7.4 7.6), is no longer valid when the functions $B(t), B_{1}(t)$ are time-dependent. As we have seen, this does not lead to any contradiction because Eqs. 7.4 7.6 have been written for a system with a fixed number of the electrons, while the solutions $\tilde{\Phi}(t)$ has been obtained for the grand canonical ensemble.

Using Eqs. 7.21 7.18 one comes to Bogolyubovde Gennes-like equations ${ }^{111}$ for the functions $u_{\mathbf{p}}(t)$ and $v_{\mathbf{p}}(t)$

$$
\left(-i \frac{\partial}{\partial t}+\hat{M}_{\mathbf{p}}^{\mathrm{mf}}(t)\right) \tilde{\phi}_{\mathbf{p}}(t)=0
$$

where

$$
\tilde{\phi}_{\mathbf{p}}(t)=\left(\begin{array}{c}
u_{\mathbf{p}}(t) \\
v_{\mathbf{p}}(t)
\end{array}\right) .
$$

It is very important that, if the periodic order parameters $B(t)$ and $B_{1}(t)$ are solutions of self-consistency equations, the functions $B\left(t-t_{0}\right)$ and $B_{1}\left(t-t_{0}\right)$ are also solutions. This means that the state is degenerate, and one should integrate over $t_{0}$ at the end of calculations to take into account the degeneracy. This procedure contrast calculations performed when studying nonequilibrium phenomena where a certain fixed time $t_{0}$ is always present marking the beginning of a process.
In the functional integral formulation of the preceding sections, the necessity of integration over $t_{0}$ simply followed from the degeneracy of the minimum of the effective action against the shift of time by $t_{0}$. In mean field theories, one should first guess a mean field solution and then check the self-consistency. If one finds a solution for any shift of $t_{0}$ (which is the case in the present situation), one should average physical quantities over $t_{0}$ at the end of calculations. From the mathematical point of view, the necessity of the integration over $t_{0}$ is more transparent in the functional integral formulation developed previously.

Of course, there is also a region of parameters of the model where a time-independent order parameter

$$
B=-i \gamma, \quad B_{1}=0,
$$

( $\gamma$ is the gap in the spectrum) corresponding to the DDW state $^{23}$ is more favorable but the time-dependent solutions are of the major interest now and we concentrate on those.

A non-trivial form of the time dependence of the wave functions $\tilde{\Phi}_{n}(t)$ (different from $\exp \left(-i E_{n} t\right)$, where $E_{n}$ is an eigenenergy) originates from the time-dependence of the order parameters $B(t)$ and $B_{1}(t)$ appearing below a critical temperature, and this is an unusual feature. However, using the fact that the functions $B(t)$ and $B_{1}(t)$ are periodic one can construct an expansion alternative to Eq. 7.4 with the help of the Floquet theorem $\frac{112}{12}$ by writing a solution $\phi_{\mathbf{p} n}(t)$ of Eq. 7.22 as

$$
\tilde{\phi}_{\mathbf{p} n}(t)=\exp \left(-i \mathcal{E}_{\mathbf{p} n} t\right) \phi_{\mathbf{p} n}(t),
$$

where $\phi_{\mathbf{p} n}(t)$ is a periodic function with the period $T_{0}$,

$$
\phi_{\mathbf{p} n}(t)=\phi_{\mathbf{p} n}\left(t+T_{0}\right),
$$

and $\mathcal{E}_{\mathbf{p} n}$ is quasienergy. The functions $\phi_{\mathbf{p} n}(t)$ and the quasienergies $\mathcal{E}_{\mathbf{p} n}$ obey the equation

$$
\left(-i \frac{\partial}{\partial t}+\hat{M}_{\mathbf{p}}^{\mathrm{mf}}(t)\right) \phi_{\mathbf{p} n}(t)=\mathcal{E}_{\mathbf{p} n} \phi_{\mathbf{p} n}(t) .
$$

Comparing Eq. 7.27) with Eq. (5.16) we see that these are the same equations for the same wave functions.

Introducing the scalar product one proves in the standard way that the functions $\phi_{\mathbf{p} n}(t)$ form the orthonormal basis,

$$
\left\{\phi_{\mathbf{p} n}^{+}(t), \phi(t)_{\mathbf{p} n^{\prime}}\right\} \equiv T_{0}^{-1} \int_{0}^{T_{0}}\left(\phi_{\mathbf{p} n}^{+}(t), \phi_{\mathbf{p} n^{\prime}}(t)\right) d t=\delta_{n n^{\prime}}
$$

The figure brackets are used here to emphasize that, in the Floquet theory, time integration is included in the scalar product. This contrasts the scalar product, Eq. 7.7), used in the conventional quantum mechanics with static Hamiltonians. For time-independent order parameters $B(t)$ and $B_{1}(t)$, the functions $\phi_{\mathbf{p} n}(t)$ are constants. The periodic dependence of the functions $\phi_{\mathbf{p} n}(t)$ on time 
contrasts the traditional time-independence of the functions $\Psi_{n}$, Eq. 7.6. As the mean field scheme is exact in the model with the Hamiltonian $\hat{H}$, Eq. (7.1), the time-dependent functions $\tilde{\Phi}_{\mathbf{p} n}(t)$, Eq. 7.16 7.27), are exact eigenfunctions of the Schrödinger equation with the Hamiltonian $\hat{H}$, Eq. 7.1.

At first glance, it looks as if the time dependence of $\tilde{\phi}_{\mathbf{p} n}(t)$ violated basic principle of thermodynamic because the conventional expression for the partition function $Z$,

$$
Z=\sum_{\text {states }} \exp \left(-E_{n} / T\right),
$$

where the sum is as usual performed over all states, does not contain time.

However, it is still possible to formulate a proper definition of the partition function $Z$ using the Floquet states 7.25 7.26. It is worth emphasizing that we do not attempt here constructing new quantum mechanics. As the main results have already been obtained in the previous sections using the functional integrals, the scheme suggested in this Section serves merely as an illustration.

Although time $t$ is explicitly present in Eqs. 7.17 . 7.27), the quantum mechanical average of an operator $\hat{A}(t)$ containing time due to dependence on the periodic functions $B(t)$ and $B_{1}(t)$ is time independent. Indeed, using Eqs. 7.25, 7.26), recalling that $B\left(t-t_{0}\right)$, $B_{1}\left(t-t_{0}\right)$ and $\phi_{\mathbf{p} n}\left(t-t_{0}\right)$ are also solutions of Eqs. 7.19, 7.20 7.22, and therefore integrating over $t_{0}$, the average of the operator $\hat{A}$ can be written as

$$
\begin{aligned}
A_{\mathbf{p}, n n} & =T_{0}^{-1} \int_{0}^{T_{0}} \tilde{\phi}_{\mathbf{p} n}^{+}\left(t-t_{0}\right) \hat{A}\left(t-t_{0}\right) \tilde{\phi}_{\mathbf{p} n}\left(t-t_{0}\right) d t_{0} \\
& =T_{0}^{-1} \int_{0}^{T_{0}} \phi_{\mathbf{p} n}^{+}\left(t-t_{0}\right) \hat{A}\left(t-t_{0}\right) \phi_{\mathbf{p} n}\left(t-t_{0}\right) d t_{0} \\
& =T_{0}^{-1} \int_{0}^{T_{0}} \phi_{\mathbf{p} n}^{+}(t) \hat{A}(t) \phi_{\mathbf{p} n}(t) d t
\end{aligned}
$$

Equation 7.30 shows that $\hat{A}_{\mathbf{p}, n n}$ is time-independent.

Actually, the Hamiltonian formalism presented in this section is a natural generalization of the conventional one. Although, the wave functions and order parameters depend on time, one can construct thermodynamics in an almost standard way. Usually, calculating the partition function $Z$ one starts with the Gibbs formula

$$
\begin{aligned}
Z & =\operatorname{Tr} \exp (-\hat{H} / T) \\
& =\sum_{n}\left(\tilde{\Psi}_{n}^{+}(t), \tilde{\Psi}_{n}(t)\right) \exp \left(-E_{n} / T\right) \\
& =\sum_{n}\left(\Psi_{n}^{+}, \Psi_{n}\right) \exp \left(-E_{n} / T\right),
\end{aligned}
$$

with the functions $\tilde{\Psi}_{n}(t)$ and $\Psi_{n}$ introduced in Eqs. 7.5 , 7.6). Eq. (7.31) contains explicitly the sum over all states. This is the standard formalism that can be used for describing the normal metal phase or the DDW phase with the order parameter given by Eq. (7.24).
Now we solve Eq. 7.21 using the Hamiltonian $\hat{H}^{\mathrm{mf}}\left(t-t_{0}\right)$, Eq. 7.17). As the Hamiltonian $\hat{H}^{\mathrm{mf}}\left(t-t_{0}\right)$ is periodic in time, we use the Floquet theorem and write the function $\tilde{\Phi}(t)$ satisfying Eq. 7.21 in the form

$$
\tilde{\Phi}_{n}(t)=\exp \left(-i \mathcal{E}_{n} t\right) \Phi_{n}(t)
$$

where $\Phi_{n}(t)$ are periodic functions with the period $T_{0}$ satisfying the equation

$$
\left(\hat{H}^{\mathrm{mf}}(t)-i \frac{\partial}{\partial t}\right) \Phi_{n}(t)=\mathcal{E}_{n} \Phi_{n}(t),
$$

and $\mathcal{E}_{n}$ is quasienergy.

It looks natural to replace $\Psi_{n}(t)$ and $E_{n}$ in Eq. (7.31) by $\Phi_{n}(t)$ and $\mathcal{E}_{n}$ and write taking into account the degeneracy with respect to the shift of time $t \rightarrow t-t_{0}$ the following formula for the partition function

$$
\begin{aligned}
Z= & T_{0}^{-1} \sum_{n} \int_{0}^{T_{0}} d t_{0} \\
& \times\left(\Phi_{n}^{+}\left(t-t_{0}\right), \Phi_{n}\left(t-t_{0}\right)\right) \exp \left(-\mathcal{E}_{n} / T\right) .
\end{aligned}
$$

Using the periodicity and the normalization, Eq. 7.28 , of the functions $\tilde{\Phi}_{n}(t)$, one comes after integration over $t_{0}$ to the following formula for the partition function

$$
Z=\sum_{n} \exp \left(-\mathcal{E}_{n} / T\right)
$$

Equation (7.35 may generalize the standard formula for the partition function, Eq. 7.31), to the case of the time-dependent wave functions. It shows that, although one obtains time-dependent order parameters and wave functions, the degeneracy with respect to the shift of time $t \rightarrow t-t_{0}$ and the necessity of the averaging over $t_{0}$ allows one to write, in particular, a reasonable formula for the partition function $Z$.

Of course, Eqs. 7.34 7.35 are just a guess. It would be interesting to prove the equivalence between such an approach and the calculations in imaginary time $\tau$ carried out in the preceding sections but this is beyond the scope of this work. At present, we merely demonstrate that the appearance of the time-dependent wave functions does not contradict thermodynamics.

Although single-time correlation functions do not depend on time, which becomes clear after integration over $t_{0}$, two or more times correlation functions can be dependent on differences of times.

\section{Why the 'no-go' theorem cannot be applied to the present scenario.}

The results obtained in the previous sections do not agree with conclusions of the 'no-go' theorem 10 , and it is worth discussing this disagreement in details. Although 
it looks at first glance that the proof of the theorem is general, it cannot be used in the situation considered in the present work.

Actually, the publication ${ }^{10}$ contains two different parts. First, the authors prove that a two-time correlation function of two arbitrary operators integrated over the volume of the system cannot have the long-range time order at zero temperature at $T=0$. The consideration of this part is based on an assumption of locality of the Hamiltonian but does not imply a specific form of the latter. The second part containing the proof for finite temperatures is different. It is based on rigorous results of Ref. ${ }^{113}$ obtained for quantum spin models. Although this part is rigorous, the results can be used for a certain class of spin models, while models of interacting fermions have not been considered at all.

The results of the present work disagree with the results of both the parts of the publication 10 and now we discuss these two parts separately.

\section{Long-range time order at $T=0$.}

The proof of the theorem is performed at zero temperature $T=0$ using a rather simple chain of inequalities applied for calculation of a two-time correlation function of two operators (Eq. (6) of Ref! ${ }^{10}$ ). The quantum mechanical average $\langle 0|\ldots| 0\rangle$ is performed using the ground state $|0\rangle$. However, the final inequality (5) of that work has been obtained assuming implicitly that the wave function of the ground state $|0\rangle$ did not depend on time. Indeed, this looked self-evident for a time-independent Hamiltonian, and the authors did not even mention that this assumption had been made. Within the method of the functional integration used in the present paper, the concept of the wave functions of the ground state was not used for calculations. However, a direct comparison becomes possible using the Hamiltonian approach developed in this section.

The notion of the order parameter and spontaneous breaking of the time-translation symmetry was not used in Ref. ${ }^{[0}$, and therefore the question about a timedependence of the wave functions of the ground state could not arise. Although all wave functions are timedependent even for static Hamiltonians due to the prefactor $\exp \left(-i E_{n} t\right)$, Eqs. 7.5, 7.6, this time-dependent prefactor cancels its complex conjugate in quantum mechanical averages.

However, if time-dependent periodic order parameters appear as a result of the spontaneous breaking of the time-translation symmetry, the eigenfunctions of the Hamiltonian acquire the Floquet-type form, Eq. (7.32). The pre-factor $\exp \left(-i \mathcal{E}_{n} t\right)$ and its complex conjugate cancel each other in the average over the ground state in this case, too, but the periodic functions $\Phi_{0}(t)$, Eq. 7.32 , are still there. Finally, the average over the ground state takes effectively a form like $\left\langle\Phi_{0}(t)|\ldots| \Phi_{0}(t)\right\rangle$ with time-dependent functions $\Phi_{0}(t)$, which invalidates the proof given in the first part of Ref. 10 .

It is worth emphasizing that we consider a grand canonical ensemble with the fixed chemical potential, while the standard form, Eq. (7.5), of the solution of the Schrödinger equation is written for a Hamiltonian with fixed number of particles. Therefore, the inequalities of Ref. 10 are correct, e.g. for a system of local spins but are not valid for the model considered here.

In order to make a direct comparison of the present results with those obtained in the first part of Ref. 10 , it is instructive to consider the correlation functions of an operator $\hat{A}$ at two different times $t_{1}$ and $t_{2}$. We can write the correlation function $C\left(t_{1}, t_{2}\right)$ in the standard way

$$
C\left(t_{1}, t_{2}\right)=\frac{U_{0}^{2}}{V^{2}}\left\langle\Phi_{0}^{*}\left|\hat{A}\left(t_{1}\right) \hat{A}\left(t_{2}\right)\right| \Phi_{0}\right\rangle,
$$

where $\Phi_{0}$ is the wave function of the ground state of the Hamiltonian $\hat{H}$, Eq. 7.1, and

$$
\hat{A}(t)=e^{i \hat{H} t} \hat{A} e^{-i \hat{H} t} .
$$

Actually, Eqs. 7.36 7.37), is the starting point of the proof given in the first part of Ref.10. At the same time, the function $C\left(t_{1}, t_{2}\right)$ coincides with the function $N\left(t_{1}-t_{2}\right)$, Eq. 5.1 , provided the operator $\hat{A}$ is chosen in the following form

$$
\hat{A}=\sum_{p}\left(c_{p}^{+} \Sigma_{2} c_{p}\right)
$$

where $c_{p}^{+}$and $c_{p}$ are the fermion operators introduced in Eq. 7.1,

$$
c_{p}(t)=e^{i \hat{H} t} c_{p} e^{-i \hat{H} t}
$$

and the Hamiltonian $\hat{H}$, Eq. 7.1, is used for the calculations.

The function $C\left(t_{1}, t_{2}\right)$, Eq. 7.36), can be rewritten in a form

$$
C\left(t_{1}, t_{2}\right)=C_{0}\left(t_{1}, t_{2}\right)+C_{1}\left(t_{1}, t_{2}\right)
$$

with

$$
\begin{aligned}
C_{0}\left(t_{1}, t_{2}\right) & =\frac{U_{0}^{2}}{V^{2}}\left(A_{00}\right)^{2} \\
C_{1}\left(t_{1}, t_{2}\right) & =\frac{U_{0}^{2}}{V^{2}} \sum_{m \neq 0} A_{0 m} A_{m 0} e^{-\left(E_{m}-E_{0}\right)\left(t_{1}-t_{2}\right)}
\end{aligned}
$$

In Eqs. 7.41, $A_{0 m}$ are matrix elements of the operator $\hat{A}$ between the ground state 0 and a state $m$. It is the correlation function $C_{1}\left(t_{1}, t_{2}\right)$ that has been estimated in Ref.10. Indeed, subtracting from the function $C_{1}\left(t_{1}, t_{2}\right)$ its value at $t_{1}=t_{2}$ and taking the absolute value we 
obtain

$$
\begin{aligned}
& \left|C_{1}\left(t_{1}, t_{2}\right)-C_{1}\left(t_{2}, t_{2}\right)\right| \\
= & \frac{2 U_{0}^{2}}{V^{2}} \sum_{m \neq 0} A_{0 m} A_{m 0} \sin \frac{\left|E_{m}-E_{0}\right|\left|t_{1}-t_{2}\right|}{2} \\
\leq & \frac{U_{0}^{2}}{V^{2}}\left|t_{1}-t_{2}\right| \sum_{m \neq 0} A_{0 m} A_{m 0}\left|E_{m}-E_{0}\right| .
\end{aligned}
$$

In the model considered here, the sum over $m$ corresponds to a sum over momenta $p$. Replacing the sum over $p$ by the integration with the help of Eq. (7.2) one can see that the inequality 7.42 agrees with the inequality (5) of Ref. ${ }^{[0,}$, although it is obtained now in a less rigorous way. However, this agreement does not mean that the time crystals are impossible because the correlation function $C_{0}\left(t_{1}, t_{2}\right)$ in Eqs. 7.41$)$ is not necessarily equal to zero. The contribution $C_{0}\left(t_{1}, t_{2}\right)$ was not considered in Ref ${ }^{10}$ at all.

The Hamiltonian $\hat{H}$ is written in such a way that the mean field theory becomes exact in the limit $V \rightarrow \infty$. This allows us to make the replacement (7.17) and follow the subsequent steps, Eqs. 7.16 7.28). The quantummechanical average $C_{00}\left(t_{1}, t_{2}\right)$ in Eq. 7.36) equals the product of the averages of the operators $\hat{A}\left(t_{1}\right)$ and $\hat{A}\left(t_{2}\right)$. We assume that the initial symmetry of the Hamiltonian $\hat{H}$ 7.1 is broken due to existence of the order parameters $B(t)$ and $B_{1}(t)$. Then, neglecting the function $C_{1}\left(t_{1}, t_{2}\right)$ we write

$$
\begin{aligned}
& C\left(t_{1}, t_{2}\right) \\
= & \frac{U_{0}^{2}}{V^{2}} \sum_{s} \sum_{p_{1}, p_{2}}\left\langle c_{p_{1}}^{+}\left(t_{1}\right) \Sigma_{2} c_{p_{1}}\left(t_{1}\right)\right\rangle_{s}\left\langle c_{p_{2}}^{+}\left(t_{2}\right) \Sigma_{2} c_{p_{2}}\left(t_{2}\right)\right\rangle_{s},
\end{aligned}
$$

where the angular brackets stand for the quantummechanical averaging over all degenerate states $s$ corresponding to the ground state. Using the self-consistency equations 7.19 , 7.20 we obtain

$$
C\left(t_{1}, t_{2}\right)=\overline{B\left(t_{1}-t_{0}\right) B\left(t_{2}-t_{0}\right)}
$$

and come finally to Eqs. 55.40 5.43. The bar in Eq. (7.44) stands for averaging over the time shift $t_{0}$. This corresponds to summation over all degenerate states.

Now it is clear at which point the present derivation has deviated from the arguments of Ref 10 . The authors of the 'no-go' theorem did not consider the spontaneous breaking of the symmetry with the formation of the timedependent order parameter and therefore missed the possibility of the periodic dependence of the two-time correlation function, Eq. 7.44).

\section{Quantum spin models.}

The 'no-go' proof presented in the second part of Ref.10 for finite temperatures is based on rigorous results of Ref. ${ }^{113}$. No doubts, the results of Ref. ${ }^{[13}$ are correct and rigorous but, as follows already from the title of that publication, the authors considered quantum spin models, which is sufficiently far away from what is considered here. So, the rigor of the arguments of that work is not an argument against the present results because the models considered there are different from the model considered here. Indeed, the scheme developed here for the fermion model simply cannot be applied to spin models. Many-body fermion models with interaction can in some cases be 'bosonized' but no general scheme making this mapping in arbitrary dimensions exists. Onedimensional models are rather an exception than a rule.

Although the arguments presented in Ref. ${ }^{[13}$ are general, a very important restriction is imposed on the models under consideration: the spin operators are local. This means that their commutators decay fast when the operators are taken at sufficiently distant points. This restriction is formulated already in the abstract and in the beginning of Sec. 3 of Ref.113. The criterion of the locality has been formulated explicitly in Sec. 2 of an earlier work by one of the authors of Ref. 114 .

In fact, a pseudospin reformulation of the Hamiltonian $\hat{H}$, Eq. 7.1), can be carried out, and one has to answer the question why the proof of Ref.113 cannot be applied to the present case. One can proceed introducing pseudospin operators $\Sigma_{i p}$ similar to those introduced by Anderson in Ref ${ }^{115}$ in the BCS model for superconductors. In the present case, the pseudospin operators are introduced as follows

$$
\begin{aligned}
& \hat{\Sigma}_{1 p}=\left(c_{p}^{1+} c_{p}^{2}+c_{p}^{2+} c_{p}^{1}\right)=c_{p}^{+} \Sigma_{1} c_{p} \\
& \hat{\Sigma}_{2 p}=-i\left(c_{p}^{1+} c_{p}^{2}-c_{p}^{2+} c_{p}^{1}\right)=c_{p}^{+} \Sigma_{2} c_{p}, \\
& \hat{\Sigma}_{3 p}=c_{p}^{1+} c_{p}^{1}-c_{p}^{2+} c_{p}^{2}=c_{p}^{+} \Sigma_{3} c_{p} .
\end{aligned}
$$

In a vector notation one writes a vector operator

$$
\hat{\boldsymbol{\Sigma}}_{p}=c_{p}^{+} \boldsymbol{\Sigma} c_{p}
$$

where $\boldsymbol{\Sigma}$ is the vector of the Pauli matrices $\Sigma_{i}, i=1,2,3$.

It is important to emphasize that the pseudospin operators $\hat{\Sigma}$ are merely a convenient computational tool and have nothing to do with real local spins.

It can easily be checked using the fermion anticommutation relations for the operators $c_{p}, c_{p}^{+}$that the operators $\hat{\Sigma}_{p}^{i}$ obey spin commutation relations

$$
\left[\hat{\Sigma}_{i p}, \hat{\Sigma}_{j p^{\prime}}\right]=2 i e_{i j k} \hat{\Sigma}_{k p^{\prime}} \delta_{p p^{\prime}}
$$

where $e_{i j k}$ is the antisymmetric tensor. The Hamiltonian $\hat{H}$, Eq. 7.1), can be rewritten in terms of the spin operators $\hat{\boldsymbol{\Sigma}}_{p}$ as

$$
\begin{aligned}
\hat{H}= & \sum_{p}\left[\varepsilon^{+}(\mathbf{p}) n_{p}+\varepsilon^{-}(\mathbf{p}) \hat{\Sigma}_{3 p}\right] \\
& -\frac{1}{4 V}\left[U_{0}\left(\sum_{p} \hat{\Sigma}_{2 p}\right)^{2}-\tilde{U}_{0}\left(\sum_{p} \hat{\Sigma}_{1 p}\right)^{2}\right],
\end{aligned}
$$


where

$$
n_{p}=c_{p}^{1+} c_{p}^{1}+c_{p}^{2+} c_{p}^{2}=c_{p}^{+} c_{p}
$$

is the density operator. As the operator $n_{p}$ commutes with $\hat{\boldsymbol{\Sigma}}_{p}$, the term with $n_{p}$ in $\hat{H}$ is not important. The most interesting correlation function that determines the long-range order in space, Eq. (7.43), takes in the pseudospin representation the form

$$
C\left(t_{1}, t_{2}\right)=\frac{U_{0}^{2}}{V^{2}} \sum_{p_{1}, p_{2}}\left\langle\hat{\Sigma}_{2 p_{1}}\left(t_{1}\right) \hat{\Sigma}_{2 p_{2}}\left(t_{2}\right)\right\rangle
$$

Eq. 7.48, is quite convenient for calculations. For example, Eqs. $5.19,5.23,5.24,5.25$ can be obtained from equations of motion for the operators $\hat{\boldsymbol{\Sigma}}_{p}$ by the quantum mechanical averaging of the operators $\hat{\boldsymbol{\Sigma}}_{p}$. The derivations are very similar to those carried out in superconductivity theory using the Anderson pseudospins 100 102.

So, the fermion model specified by the Hamiltonian $\hat{H}$, Eq. 7.1), has been exactly rewritten in terms of the pseudospin operators $\hat{\Sigma}_{p}$. The choice of these operators is unambiguous because we are interested in studying the long-range order in both space and time described by the function $C\left(t_{1}, t_{2}\right)$, Eqs. 7.36 7.39). Equation (7.50) allows one to use these operators directly for the calculation of the correlation function of interest. Attempts to find other spin operators do not make a sense because the function $C\left(t_{1}, t_{2}\right)$, Eq. 7.36), would be considerably more complicated in terms of the other operators, while a correlation function of several those operators will not be related to the long-range order. One should really use the operators $\hat{\Sigma}_{p}$, Eqs. 7.45 , and we have a well defined model, Eq. 7.48, for that.

Can the rigorous estimates of Ref ${ }^{113}$ repeated for the model, Eq. (7.48), invalidate the hope of obtaining the thermodynamic quantum time-space crystal? The answer is definitely 'no' because the operators $\hat{\Sigma}$ written in real space are not local, and the arguments of Ref. 113 cannot be repeated.

Indeed, writing the operators in space points as

$$
\hat{\Sigma}_{i}(\mathbf{r})=\frac{1}{V} \sum_{\mathbf{p}} \hat{\Sigma}_{i \mathbf{p}} e^{i \mathbf{p r}}
$$

we obtain for the Hamiltonian $\hat{H}$, Eq. 7.48, the following formula (omitting the term with $n_{p}$ )

$\hat{H}=V\left[\left(\varepsilon^{-}(-i \nabla) \hat{\Sigma}_{3}(\mathbf{r})\right)_{\mathbf{r}=0}-\frac{1}{4}\left(U_{0} \hat{\Sigma}_{2}^{2}(0)-\tilde{U}_{0} \hat{\Sigma}_{1}^{2}(0)\right)\right]$,

while the correlation function $C\left(t_{1}, t_{2}\right)$ takes the form

$$
C\left(t_{1}, t_{2}\right)=-U_{0}^{2}\left\langle\hat{\Sigma}_{2}\left(t_{1}, 0\right) \hat{\Sigma}_{2}\left(t_{2}, 0\right)\right\rangle
$$

where

$$
\hat{\Sigma}_{i}(t, \mathbf{r})=e^{i \hat{H} t} \hat{\Sigma}_{i}(\mathbf{r}) e^{-i \hat{H} t}
$$

The Hamiltonian $\hat{H}$, Eq. 7.52, is very different from those for local spin models. Moreover, we write the commutation relation for the pseudospin operators $\Sigma_{i}(\mathbf{r})$ and $\Sigma_{j}\left(\mathbf{r}^{\prime}\right)$ at different space points $\mathbf{r}$ and $\mathbf{r}^{\prime}$ as

$$
\left[\hat{\Sigma}_{i}(\mathbf{r}), \hat{\Sigma}_{j}\left(\mathbf{r}^{\prime}\right)\right]=\frac{1}{V^{2}} \sum_{p, p^{\prime}} e^{i\left(\mathbf{p r}+\mathbf{p}^{\prime} \mathbf{r}^{\prime}\right)}\left[\hat{\Sigma}_{i p}, \hat{\Sigma}_{j p^{\prime}}\right]
$$

Using Eq. 7.47 we obtain

$$
\begin{aligned}
{\left[\hat{\Sigma}_{i}(\mathbf{r}), \hat{\Sigma}_{j}\left(\mathbf{r}^{\prime}\right)\right] } & =\frac{2 i e_{i j k}}{V^{2}} \sum_{p} e^{i \mathbf{p}\left(\mathbf{r}+\mathbf{r}^{\prime}\right)} \hat{\Sigma}_{k p} \\
& =\frac{2 i e_{i j k}}{V} \hat{\Sigma}_{k}\left(\mathbf{r}+\mathbf{r}^{\prime}\right)
\end{aligned}
$$

One can see from Eq. 7.56 that the commutator of the pseudospins $\hat{\Sigma}_{i}(\mathbf{r})$ at two different points $\mathbf{r}$ and $\mathbf{r}^{\prime}$ does not vanish in the limit $\left|\mathbf{r}-\mathbf{r}^{\prime}\right| \rightarrow \infty$. Presence of the pre-factor $V^{-1}$ in Eq. 7.56 does not make the operators $\hat{\Sigma}_{i}(\mathbf{r})$ local even in the limit $V \rightarrow \infty$ because this pre-factor is compensated in equations of motion by the factor $V$ in the Hamiltonian $\hat{H}$, Eq. 7.52.

All this means that the operators $\hat{\Sigma}(t, \mathbf{r})$ are not local, and applying the results of the work 113 based on the assumption of the locality of the spins to the model considered here is not justified. As the BCS model of superconductivity can also be reformulated in terms of the similar Anderson pseudospins, one cannot use the bounds of Ref. 113 for studying properties of superconductivity either. In particular, amplitude (Higgs) modes $97-103$ are not known in systems of local spins. So, the local quantum spin models cannot in general describe electronelectron or electron-hole pairing in fermion models with interaction, and the bounds obtained in Ref. ${ }^{113}$ cannot be applied to the model considered here.

\section{Concluding remarks to Section VII.}

We conclude this Section with the statement that the 'no-go' theorem of Ref ${ }^{10}$ is not applicable to the phenomenon of the thermodynamic quantum time-space crystal considered in the present work.

Strictly speaking, the results are obtained using the Hamiltonian $\hat{H}$ with a somewhat special interaction. Although the original electron-electron interaction is shortranged, the form of the interaction in Eq. (7.1) corresponds to an 'infinite-range' interaction of the electronhole pairs. However, mean field theories for systems of interacting electrons are well justified for small ratios of the order parameter to the Fermi energy, which holds in most cases.

The fact that one can use the wave functions in the standard form of Eq. 7.5 is applicable for models with a fixed number of particles. At the same time, the grand canonical enesemble of interacting electrons is discussed in the present work, and the wave functions have a more complicated form that cannot be reduced to Eq. 7.5 . 
The spontaneous breaking of the symmetry occurs in the grand canonical ensemble. So, the non-trivial timedependent form of the wave functions obtained here does not contradict the standard form of the wave functions, Eq. 7.5.

The consideration of the first part of Ref ${ }^{10}$ was based on the assumption of the locality of the Hamiltonian. The authors of Ref. 10 write explicitly that their proof is not applicable to models with an infinite-range interactions. Although the electron-electron interaction in the Hamiltonian $\hat{H}$, Eq. 7.1, is short ranged, it describes effectively an 'infinite range' interaction of elecron-hole pairs. Could it mean that the results obtained in the present paper are not general and are specific to the considered model only? In this case, this would mean that taking a more realistic interaction would destroy the long-range time oscillations, and the results obtained here would not be as interesting.

Fortunately, it does not seem to be so. Such a scenario would be possible for a spin model with an infiniterange interaction. Indeed, according to the estimates of Ref. 10 that are definitely correct for the spin systems, making the radius of the interaction finite would destroy the long-time oscillations but does not apply to the infinite range interaction. However, the infinite range of the interaction would lead to, e.g., ferromagnetic or antiferomagnetic states rather to time crystals. I do not see any chance to obtain the TQTC with a time-dependent order parameter in the spin models, and they are not considered here. The situation with the 'infinite-range' interaction of the electron-hole pairs in Eq. (7.1) is completely different because the electron-electron interaction is short-ranged and the Hamiltonian $\hat{H}$ is local.

This property can easily be seen using an important estimate for local Hamiltonians written on top of p. 3 in the proof of Ref! 10 ,

$$
[\hat{A}[\hat{H}, \hat{A}]] \propto V .
$$

The proportionality to the volume $V$ instead of $V^{2}$ should indicate according to Ref. 10 the absence of the time crystal behavior. The proportionality to $V^{2}$ is possible for the infinite-range interactions in the spin models.

On the other hand, it is not difficult to calculate the same commutator for the Hamiltonian $\hat{H}$, Eqs. 7.1 . 7.48, and operator $\hat{A}$, Eq. 7.38). The computation is easy with the help of the pseudospins $\hat{\Sigma}$, Eqs. 7.45 . 7.47), and one obtains

$$
\begin{aligned}
{[\hat{A}[\hat{H}, \hat{A}]]=} & -4 \sum_{p} \varepsilon^{-}(\mathbf{p}) \hat{\Sigma}_{3 p} \\
& +\frac{8 \tilde{U}_{0}}{V} \sum_{p_{1} \neq p_{2}}\left(\hat{\Sigma}_{3 p_{1}} \hat{\Sigma}_{3 p_{2}}-\hat{\Sigma}_{1 p_{1}} \hat{\Sigma}_{1 p_{2}}\right) .
\end{aligned}
$$

Standard replacement of the sum over the momenta by the integral, Eq. (7.2), leads to the conclusion that the estimate, Eq. (7.57), holds in our case, too. This means that the Hamiltonian $\hat{H}$, Eq. 7.1, with the 'infiniterange' interactions of the elecron-hole pairs should be classified as a short-range Hamiltonian in the proof of Ref.10. Existence of the TQTC is a consequence of the breaking of the time-translation symmetry, and the 'infinite-range' interaction of the electron-hole pairs is helpful but not necessary for the latter phenomenon. A more general form of the electron-electron interaction in the Hamiltonian $\hat{H}$ cannot change the estimate 7.57 because the interaction is short-ranged anyway.

\section{HOW TO OBSERVE THE TIME-SPACE CRYSTAL EXPERIMENTALLY?}

As the average order parameter $B(t)$ equals zero, Eq. 5.37), one cannot expect oscillating quantities like, e.g., currents. The oscillations should be seen in two-times correlation functions like $N(t)$, Eqs. 5.1, 5.40, 5.43). Of course, possibility of an experimental observation depends on systems that can be described by the action, Eqs. 2.1 2.11, 2.13 2.15. Apparently, one can find various models exhibiting the time crystal behavior. However, since the action, Eqs. 2.1 2.11, 2.13, 2.15, is suggested here for description of cuprates and one can expect correlations of magnetic moments oscillating in time and space (in contrast to static magnetic moments in DDW theories), the polarized neutron spectroscopy can be a proper tool. In this case, the Fourier-transform of the function $N(t)$ determines directly the cross-section of the inelastic scattering. It is important that the magnetic moments are basically perpendicular to the planes, which can help to distinguish them from the antiferromagnetic spin excitations at $(\pi, \pi)$. Calculating the Fourier transform $N(\omega)$ of the function $N(t)$, Eq. (5.40), and comparing it with the one for the time-independent DDW state $2 \pi \gamma^{2} \delta(\omega)$ for the same model one can write at low temperatures the ratio of the experimental responses at $(\pi, \pi)$ for these two states as

$$
\chi(\omega, \mathbf{q})=\chi_{0} \sum_{n=1}^{\infty} f_{n}^{2} \delta(\omega-2 n \gamma) \delta\left(\mathbf{q}-\mathbf{Q}_{A F}\right),
$$

where $f_{n}$ is determined by Eq. (5.41), and $\chi_{0}$ determines the response $\chi_{D D W}$ of the DDW state, $\chi_{D D W}(\omega)=$ $\chi_{0} \delta(\omega)$.

Actually, anisotropic magnetic $(\pi, \pi)$ excitations have been observed $\frac{116}{10} \mathrm{YBa}_{2} \mathrm{Cu}_{3} \mathrm{O}_{6.9}$. The response $\chi_{c}(\omega, \mathbf{q})$ perpendicular to planes with wave vector $\mathbf{q}=(1.5, k, 1.73)$ had a pronounced peak at $k=0.5$ at $\omega=26 \mathrm{meV}$ at temperature $94 K$, while this peak was suppressed at $10 K$, which was below the superconducting temperature $T_{c}$. At the same time, the parallel component $\chi_{a / b}$ was not so sensitive to temperature. Using the results obtained in the present paper one might argue that the peak in the susceptibility $\chi_{c}(\omega, \mathbf{q})$ was due the oscillations of magnetic moments. Within this picture, the time-space crystal state had to be suppressed by the superconductivity. 
Being quite general, the model specified by Eqs. 2.13 . 2.15 may also be applied to other systems and one should design proper experiments for each case.

\section{DISCUSSION.}

The main result of the present study is that the quantum time crystals may exist as a thermodynamically stable state in macroscopic systems even in the limit of infinite volume, $V \rightarrow \infty$. The non-decaying oscillations do not lead to an oscillating behavior of classical quantities and the energy is conserved. The oscillations show up in correlation functions of several times and can in principle be observed in e.g. quantum scattering experiments. The order parameter of the thermodynamic quantum time-space crystals is periodic in both real and imaginary times as well as in space but its average over the phases of the oscillations vanish.

The procedure of the averaging over the phase of the oscillations is equivalent to a new quantum description in terms of the operator order parameter. In this picture, the oscillating behavior of correlation functions originates from virtual transitions between states of an oscillator. The distance between the energy levels equals the energy of breaking the electron-hole pairs and does not decrease in the limit of infinite volume, $V \rightarrow \infty$.

All calculations have been carried out in the limit of low temperatures for a simplified version of the spinfermion model with overlapping hot spots relevant for the superconducting cuprates. In this simplified version, the electron-electron interaction is written in a form of an infinite-range interaction of electron-hole pairs. This is a standard approximation used for studying new phase transitions in many-body electron systems first suggested by Bardeen-Cooper-Schrieffer in their fundamental paper on theory of superconductivity 88 . This type of simplified models allows one to solve the problem exactly and gives results that could be obtained from more general models using a proper mean field approximation.

The electron-electron interaction of the original model is usually short-ranged, and its explicit form is irrelevant for the phenomena studied by this method. The possibility of using the mean field approximation in the electron systems is different from the one used in, e.g., spin models. In the latter case, the mean field approximation is usually justified by using a long-range interaction between the spins, while in the electron models one needs merely a small ratio of the order parameter to the Fermi energy. Formation of charge density wave (CDW) and many other phase transitions have been predicted and described in this way without assuming any special longrange interaction.

Of course, one has to investigate fluctuations near the mean field solution. Actually, it is possible to calculate the fluctuations in the same way as it is usually done when studying new phases in electron systems. In practical terms, one can perform Hubbard-Stratonovich de- coupling of the interaction in the original Lagrangian, Eqs. 2.3 2.11), and integrate out the fermion fields $\chi, \chi^{*}$. Then, one obtains a free energy functional of the auxiliary fields $b$ and $b_{1}$ like the ones written in Eq. (3.10). Generally, the fields $b$ and $b_{1}$ are functions of not only time but also of coordinates. Following this scheme one finds the minimum of the functional $\mathcal{F}\left[b, b_{1}\right]$ and expands in the deviations $\delta b$ and $\delta b_{1}$ from the functions $b^{(0)}$ and $b_{1}^{(0)}$ at the minimum. The functions $b^{(0)}$ and $b_{1}^{(0)}$ are just solutions of the mean field equations.

As a result, one obtains a positive-definite quadratic form. Contribution of higher order terms in $\delta b$ and $\delta b_{1}$ can be taken into account by expansion in these variables and calculation of Gaussian integrals. In low dimensions, some of these contributions can be divergent, and one should use more sophisticated schemes of calculations. However, in quasi-two and three-dimensional systems the corrections to the mean field solutions are convergent, and unless they are very large the mean field theory is a reasonable approximation. Preliminary consideration for the present model shows that expansions near the minimum of the free energy functional lead to convergent integrals and they are not very large. A more detailed investigation of this problem is left for future but for now the mean field theory does not look a bad approximation.

It is important to emphasize that a thermodynamically stable time-crystal state corresponding to the minimum of the free energy has been obtained for the first time. All previous works on time crystals have been performed for non-equilibrium systems, which means that they were not at a minimum. Therefore fluctuations could rather easily drive the systems away from the initial state and destroy mean field solutions.

An important problem that awaits its resolution is finding the exact minimum of the free energy functional $\mathcal{F}\left[b, b_{1}\right]$, Eq. 3.10 . Although the scheme of the calculations used for drawing Figs. 3 is plausible, a more precise computation is certainly needed. Of course, one can do this numerically but a more detailed analytical investigation would be helpful. Alternatively, one could study Eqs. 55.19, 5.20, 5.23 5.25 for the pseudospins $\mathbf{S}_{\mathbf{p}}(t)$. It is well-known that corresponding equations for superconductors are integrable ${ }^{100} \frac{102}{10}$, and analyzing the possibility of the integrability of Eqs. $5.19,5.20,5.23$. 5.25 would be of a great interest.

One of the most exciting concepts of theoretical condensed matter and high energy physics is the spontaneous breaking of symmetry. According to this concept, original symmetries of the Hamiltonian can change below the phase transition due to formation of the order parameter. For example, in the BCS theory the effective Hamiltonian does not conserve the particle number, appearance of CDW breaks the space-translation symmetry, etc. The thermodynamic quantum time crystal (TQTC) proposed here is conceptually very similar to CDW. The main difference is that now the time-translation symmetry is broken instead of the space-translation one. Both the phenomena are described within similar computa- 
tional schemes (actually, in the present model both the time and space symmetries are broken and one can speak of time-space crystal).

The inapplicability of the 'no-go' theorem of Ref ${ }^{10}$ to the results obtained here follows from the fact that the authors did not imply the possibility of the spontaneous breaking of the time-translation symmetry in their proof. Their additional arguments based on the use of known rigorous results for models of local spins cannot be applied to the electron models considered here.

Although study of the time crystals is already a mature field of research and a lot of interesting results have been obtained using various models and techniques, TQTC is really a new phenomenon because, in all the previous works, non-equilibrium phenomena were considered. Although the time crystal as a thermodynamic state has been proposed by Wilczek in his pioneering work ${ }^{2}$, a subsequent study has shown that his state was not in thermodynamic equilibrium. As the 'no-go' theorem of Ref!10 has been widely accepted, no attempts to obtain a thermodynamically stable time crystal have been undertaken since then. The fact that the time crystal obtained here is thermodynamically stable makes it different from all time crystals obtained previously. One might argue that already a trivial 2-level system like a spin in a magnetic field gives a similar 2-time correlation function $N(t) \underline{10}$. However, it is demonstrated here that this effect can exist in a macroscopic systems in the limit $V \rightarrow \infty$ in contrast to the statement of that work. The fact that a phase transition effectively results in the formation of energy levels with non-vanishing in the limit $V \rightarrow \infty$ level spacing and quantum oscillations between the levels can in principle be observed in physical quantities, is non-trivial and does not have any analogs.

The novelty and importance of the TQTC in macroscopic samples and its difference with respect to the nonequilibrium time-crystals can be understood in simple terms making a qualitative comparison with three different types of electron systems in a magnetic field with nondecaying currents: 1) an electron in an atom subjected to a magnetic field, 2) ideal conductor in a magnetic field, and 3) superconductor in a magnetic field.

In case 1) a non-decaying electron current flows around the atom and generates a constant magnetic moment.

In case 2 ) the applied magnetic field can also induce non-decaying diamagnetic currents and a diamagnetic moment but these quantities are sensitive to impurities phonons, etc. They depend also on the pre-history because one obtains different results using different experiments. The diamagnetic currents are induced when one applies the magnetic field at low temperatures. On the other hand, one does not obtain any currents if one starts with the system in the magnetic field at high temperatures, such that the current has vanished due to the scattering of the electrons on phonons. Then, one cools down the sample but does not obtain any current. The dependence on the pre-history is typical for non-equilibrium phenomena. Of course, one can maintain the currents applying an oscillating magnetic field that would serve as driving force.

At last, in case 3) one has a genuine thermodynamic state because, as soon as the applied magnetic field stops changing, the current evolves to a constant value that does not depend on the pre-history (Meissner effect). There are plenty of other interesting effects distinguishing the superconductors from the ideal conductors. The superconductors are characterized by the quantum coherence all over the sample and arise as a result of the spontaneous breaking of the symmetry.

It is clear why the phenomena 1)-3) are different. The phenomenon 1) is characterized by a constant value of the current but is microscopic. Both the phenomena 2) and 3) are macroscopic but 2) is a non-equilibrium phenomenon, while 3) is a thermodynamic quantum manybody state that appears as the result of the spontaneous breaking of the symmetry. We see on this example that although a non-decaying current can exist in all these systems, their nature is different, and one should study additionally other physical quantities to understand what phenomenon one deals with.

Now we can understand easily the difference between the three different types of the time-crystal-like states discussed here. The microscopic 2-level system showing the time-oscillations is just trivial, and there is no sense to call it 'time crystals'. The non-equilibrium timecrystals can be very non-trivial but they imply driving forces or they can be very sensitive to various perturbations, etc. In contrast, TQTC is a genuine thermodynamic state because the oscillations of the two-time correlation functions $N(t)$ do not depend on time at all, and this dependence is not sensitive to impurities or other static perturbations. Fluctuations are also less important in TQTC than in systems out of equilibrium, and the mean field approximation is better justified. In a certain sense, TQTC is an analog of the superconductors but the analogy to CDW is more direct.

The correlation functions $N(t)$ can, in principle, be experimentally observed in scattering experiments, and study of the properties of the TQTC looks interesting and important. The form of the two-band Hamiltonian, Eq. (7.1), is quite general and one can anticipate applications to other materials and devices. The non-decaying oscillations is a very important property for qubits and one might think of interesting applications.

Description of phase transitions between the timespace crystal state and normal metal or the state with the time-independent order parameter can also be of a great interest because they definitely differ from known phase transitions.

The thermodynamic quantum time-space crystal may be a good candidate for the still mysterious pseudogap state in superconducting cuprates. In many respects, its properties like breaking time reversal symmetry, gap in the electron spectrum, etc, resemble those of the DDW state. At the same time, no static magnetic moments oscillating with the antiferromagnetic vector $(\pi, \pi)$ have 
been observed so far in agreement with the predictions for the thermodynamic time-space crystal. Instead, the present results show that correlations of the magnetic moments oscillate in time and can in principle be studied in experiments with polarized inelastic neutron scattering.

\section{ACKNOWLEDGMENTS}

I would like to thank S.I. Mukhin, B.Z. Spivak, P.A. Volkov, G.E. Volovik, and P.B. Wiegmann for useful discussions.

Financial support of Deutsche Forschungsgemeinschaft (Projekt EF 11/10-1) and of the Ministry of Science and Higher Education of the Russian Federation in the framework of Increase Competitiveness Program of NUST "MISiS" (Nr. K2-2017-085") is greatly appreciated.

\section{APPENDIX.}

\section{Appendix A: Final formulas for the free energy of instantons and antiinstantons.}

The integrals 4.14, 4.17 4.20 can be simplified changing the variables of the integration as

$$
\begin{aligned}
p_{x} & = \pm p \sqrt{\frac{1+u}{2}}, p_{y}= \pm p \sqrt{\frac{1-u}{2}} \\
E & =\varepsilon^{-}(\mathbf{p})=\frac{\alpha-\beta}{2} p^{2} u+P
\end{aligned}
$$

with $-1<u<1$. Then, the integral $R$ for any nonsingular function $f(E)$,

$$
R=\int f\left(\varepsilon^{-}(\mathbf{p})\right) \frac{d \mathbf{p}}{(2 \pi)^{2}}
$$

can be written in the form

$R=\frac{2}{(2 \pi)^{2}} \frac{1}{\alpha+\beta} \int_{-1}^{1}\left[\int_{P(T)}^{P(T)+\Lambda u} f(E) d E\right] \frac{1}{u} \frac{1}{\sqrt{1-u^{2}}} d u$

Further, the integral over $u$ can be transformed integrating by parts and we reduce $R$, Eq. $\mathrm{A} 2$, to the following integral over one variable

$$
R=\frac{2}{(2 \pi)^{2}} \frac{\Lambda}{\alpha+\beta} \int_{-1}^{1} f(P(T)-u \Lambda) \ln \frac{\sqrt{1-u^{2}}+1}{|u|} d u
$$

which simplifies considerably the original integration over p.
Finally, using parameters $\bar{P}=P / \gamma, \bar{\Lambda}=\Lambda / \gamma$ we write the free energy $\Delta F$ as

Herein,

$$
\frac{\Delta F}{V T}=\frac{1}{\pi^{2}} \frac{m \Lambda}{\alpha+\beta}\left(s_{0}+s_{i n t}\right) .
$$

$$
\begin{aligned}
s_{0}= & 2 \int_{-1}^{1}\left[\ln \frac{\sqrt{(x-y u)^{2}+1}+1}{|x-y u|}-\frac{1}{\sqrt{(x-y u)^{2}+1}}\right] \\
& \times \ln \frac{\sqrt{1-u^{2}}+1}{|u|} d u,
\end{aligned}
$$

and

$$
\begin{aligned}
s_{\text {int }}= & -\frac{I_{0}}{2} \int_{0}^{1}\left[K_{0}+\frac{1}{4}\left(K_{1}-\frac{M^{2}}{L}\right)\left(1-v^{2}\right)^{2}\right]^{-1} \\
& \times\left(1-v^{2}\right) d v
\end{aligned}
$$

where

$$
\begin{aligned}
K_{0}= & \left(1+\frac{U_{0}}{\tilde{U}_{0}}\right) \int_{-1}^{1} \frac{1}{\sqrt{(x-y u)^{2}+1}} \ln \frac{\sqrt{1-u^{2}}+1}{|u|} d u, \\
L= & \int_{-1}^{1} \frac{1}{\left((x-y u)^{2}+1\right)^{3 / 2}} \ln \frac{\sqrt{1-u^{2}}+1}{|u|} d u, \\
M= & \int_{-1}^{1} \frac{\left((x-y u)^{2}+\frac{(1-k)^{2}}{4}\right)\left((x-y u)^{2}+1\right)^{3 / 2}}{\left(\frac{\sqrt{1-u^{2}}+1}{|u|} d u,\right.} \\
& \times \ln \frac{1}{K_{1}=} \\
& \int \frac{\left.(x-y u)^{2}+1\right)^{3 / 2}\left((x-y u)^{2}+\frac{(1-k)^{2}}{4}\right)}{\left(\frac{\sqrt{1-u^{2}}+1}{|u|} d u,\right.} \\
& \times \ln \frac{1}{(x)}
\end{aligned}
$$

and

$$
\begin{aligned}
I_{0}= & {\left[\int \frac{\operatorname{sgn}(x-y u)}{\sqrt{(x-y u)^{2}+\frac{(1-k)^{2}}{4}}} \frac{1}{\sqrt{(x-y u)^{2}+1}}\right.} \\
& \left.\times \ln \frac{\sqrt{1-u^{2}}+1}{|u|} d u\right]^{2} .
\end{aligned}
$$

Equation 3.17 that determines the gap $\gamma$ takes the form

$$
\frac{\alpha+\beta}{U_{0}}=\frac{1}{2 \pi^{2}} \int_{-1}^{1} \frac{y}{\sqrt{(x-y u)^{2}+1}} \ln \frac{\sqrt{1-u^{2}}+1}{|u|} d u
$$

Equations A6, A7, A8 have been used for computation of the surfaces in Figs. 3(a-e). 
1 L.D. Landau and Lifshitz, Course of Theoretical Physics, v.5 Statistical Physics, Pergamon Press, Oxford (1980).

2 F. Wilczek, Quantum time crystals, Phys. Rev. Lett. 109, 160401 (2012).

3 P. Bruno, "Comment on quantum time crystals", Phys. Rev. Lett. 110, 118901 (2013).

${ }^{4}$ F. Wilczek, Quantum time crystals, "Reply on Comment on quantum time crystals", Phys. Rev.Lett. 110, 118902 (2013).

5 T. Li, Z.-X. Gong, Z.-Q. Yin, H. T. Quan, X. Yin, P. Zhang, L.-M. Duan, and X. Zhang, Space-time crystals of trapped ions, Phys. Rev. Lett. 109, 163001 (2012).

${ }^{6}$ P. Bruno, Comment on space-time crystals of trapped ions, Phys. Rev. Lett. 111, 029301 (2013).

7 P. Bruno, Impossibility of spontaneously rotating time crystals: A no-go theorem, Phys. Rev. Lett. 111, 070402 (2013).

8 P. Nozieres, Time crystals: Can diamagnetic currents drive a charge density wave into rotation? Europhys. Lett. 103, 57008 (2013).

9 F. Wilczek, Superfluidity and space-time translation symmetry breaking, Phys. Rev. Lett. 111, 250402 (2013).

${ }^{10}$ H. Watanabe, and M.Oshikawa, Absence of quantum time crystals, Phys. Rev. Lett. 114, 251603 (2015).

$11 \mathrm{G}$. Volovik, On the broken time translation symmetry in macroscopic systems: Precessing states and off-diagonal long-range order, JETP Lett. 98, 491 (2013).

12 K. Sacha, Modeling spontaneous breaking of timetranslation symmetry, Phys. Rev. A 91, 033617 (2015).

13 V. Khemani, A. Lazarides, R. Moessner, and S.L. Sondhi, Phase structure of driven quantum systems. Phys. Rev. Lett. 116, 250401 (2016).

${ }^{14}$ C. W. von Keyserlingk, V. Khemani, and S. L. Sondhi, Absolute stability and spatiotemporal long-range order in Floquet systems, Phys. Rev. B 94, 085112 (2016).

15 D.V. Else, B. Bauer, and C. Nayak, Floquet time crystals, Phys. Rev. Lett. 117, 090402 (2016).

16 N.Y. Yao, A.C. Potter, I.-D. Potirniche, and A. Vishwanath, Discrete Time Crystals: Rigidity, criticality, and realizations, Phys. Rev. Lett. 118, 030401 (2017).

17 S. Autti, V.B. Eltsov, and G. E. Volovik, Observation of a time quasicrystal and its transition to a superfluid time crystal, Phys. Rev. Lett. 120, 215301 (2018).

18 J. Zhang, P.W. Hess, A. Kyprianidis, P. Becker, A. Lee, J. Smith, G. Pagano, I.D. Potirniche, A.C. Potter, A. Vishwanath, N.Y. Yao, and C. Monroe, Observation of a discrete time crystal, Nature 543, 217-220 (2017).

19 S. Choi, J. Choi R. Landig, G. Kucsko, H. Zhou, J. Isoya, F. Jelezko, S. Onoda, H. Sumiya, V. Khemani, C. von Keyserlingk, N.Y. Yao, E. Demler E, and M.D. Lukin, Observation of discrete time-crystalline order in a disordered dipolar many-body system, Nature 543, 221 (2017).

20 K.B. Efetov, Thermodynamic quantum time crystal, arXiv: 1902.07520

21 P.A. Volkov and K.B. Efetov, Spin-fermion model with overlapping hot spots and charge modulation in cuprates, Phys. Rev. B 93, 085131 (2016).

22 P.A. Volkov and K.B. Efetov, Overlapping hot spots and charge modulation in cuprates, J. Supercond. Nov. Mag., 29, 1069 (2016).

23 P.A. Volkov and K.B. Efetov, Charge and current or- ders in the spin-fermion model with overlapping hot spots. Phys. Rev. B 97, 165125 (2018).

24 T. Timusk and B. Statt, The pseudogap in hightemperature superconductors: an experimental survey, Rep. Prog. Phys. 62, 61 (1999).

25 M.R. Norman, D. Pines, and C. Kallin, The pseudogap: friend or foe of high Tc ? Advances in Physics, 54, 715 (2005).

26 M. Hashimoto, I.M. Vishik, Rui-Hua He, T. Devereaux, and T. P. Zhi-Xun Shen, Energy gaps in high-transitiontemperature cuprate superconductors, Nat. Phys. 10, 483 (2014)

27 W. W. Warren Jr, R.E. Walstedt, J.F. Brennert, R.J. Cava, R. Tycko, R.F. Bell, and G. Dabbagh, Cu spin dynamics and superconducting precursor effects in planes above $\mathrm{Tc}_{c}$ in $\mathrm{YBa}_{2} \mathrm{Cu}_{3} \mathrm{O}_{6.7}$, Phys. Rev. Lett. 62, 1193 (1989).

${ }^{28} \mathrm{H}$. Alloul, T. Ohno, and P. Mendels, ${ }^{89} Y$ NMR evidence for a fermi-liquid behavior in $\mathrm{YBa}_{2} \mathrm{Cu}_{3} \mathrm{O}_{6+x}$, Phys. Rev. Lett. 63, 1700 (1989).

29 A. Damascelli, Z. Hussain, and Z.-X. Shen, Angle-resolved photoemission studies of the cuprate superconductors, Rev. Mod. Phys. 75, 473 (2003).

30 S.Benhabib, A. Sacuto, M. Civelli, I. Paul, M. Cazayous, Y. Gallais, M.-A. Measson, R. D. Zhong, J. Schneeloch, G. D. Gu, D. Colson, and A. Forget, Collapse of the normal-state pseudogap at a Lifshitz transition in the $\mathrm{Bi}_{2} \mathrm{Sr}_{2} \mathrm{CaCu}_{2} \mathrm{O}_{8+\delta}$ cuprate superconductor, Phys. Rev. Lett. 114, 147001 (2015).

31 B. Loret, S. Sakai, S. Benhabib, Y. Gallais, M. Cazayous, M. A. Measson, R. D. Zhong, J. Schneeloch, G. D. $\mathrm{Gu}$, A. Forget, D. Colson, I. Paul, M. Civelli, and A. Sacuto, Vertical temperature boundary of the pseudogap under the superconducting dome in the phase diagram of $\mathrm{Bi}_{2} \mathrm{Sr}_{2} \mathrm{CaCu}_{2} \mathrm{O}_{8+\delta}$, Phys. Rev. B 96, 094525 (2017).

32 Y. Kohsaka, C. Taylor, K. Fujita, A. Schmidt, C. Lupien, T. Hanaguri, M. Azuma, M. Taka-no, H. Eisaki, H. Takagi, S. Uchida, and J.C. Davis, An intrinsic bond-centered electronic glass with unidirectional domains in underdoped cuprates, Science 315, 1380, (2007).

33 M.J. Lawler, K. Fujita, J. Lee, A.R. Schmidt, Y. Kohsaka, C. K. Kim, H. Eisaki, S. Uchida, J. C. Davis, J. P. Sethna, and E.-A. Kim, Intra-unit-cell electronic nematicity of the high-Tc copper-oxide pseudogap states, Nature (London) 466, 347 (2010).

34 R. Daou, J. Chang, D. LeBoeuf, O. Cyr-Choiniere, F. Laliberte, N. Doiron-Leyraud, B. J. Ramshaw, R. Liang, D. A. Bonn, W. N. Hardy, and L. Taillefer, Broken rotational symmetry in the pseudogap phase of a high-Tc superconductor, Nature 463, 519 (2007).

35 O. Cyr-Choiniere, G. Grissonnanche, S. Badoux, J. Day, D. A. Bonn, W. N. Hardy, R. Liang, N. Doiron-Leyraud, and L. Taillefer, Two types of nematicity in the phase diagram of the cuprate superconductor, $\mathrm{YBa}_{2} \mathrm{Cu}_{3} \mathrm{O}_{y}$, Phys. Rev. B 92, 224502 (2015).

36 Y. Sato, S. Kasahara, H. Murayama, Y. Kasahara, E.-G. Moon, T. Nishizaki, T. Loew, J. Porras, B. Keimer, T. Shibauchi, and Y. Matsuda, Thermodynamic evidence for a nematic phase transition at the onset of the pseudogap in $\mathrm{YBa}_{2} \mathrm{Cu}_{3} \mathrm{O}_{y}$, Nat. Phys. 13, 1074 (2017).

37 L. Zhao, C.A. Belvin, R. Liang, D.A. Bonn, W. N. 
Hardy, N. P. Armitage, and D. Hsieh, A global inversionsymmetry-broken phase inside the pseudogap region of YBa2Cu3Oy, Nat. Phys. 13, 250 (2017).

38 B. Fauque, Y. Sidis, V. Hinkov, S. Pailhes, C. T. Lin, X. Chaud, and P. Bourges, Magnetic order in the pseudogap phase of high-Tc superconductors, Phys. Rev. Lett. 96, 197001 (2006).

39 Y. Sidis and P. Bourges, Evidence for intra-unit-cell magnetic order in the pseudo-gap state of high-Tc cuprates, J. Phys.: Conf. Ser. 449, 012012 (2013).

40 L. Mangin-Thro, Y. Sidis, A. Wildes, and P. Bourges, Intra-unit-cell magnetic correlations near optimal doping in $\mathrm{YBa}_{2} \mathrm{Cu}_{3} \mathrm{O}_{6.85}$, Nat. Commun. 6, 7705 (2015).

41 L. Mangin-Thro, Y. Li, Y. Sidis, and P. Bourges, $a-$ $b$ Anisotropy of the intra-unit-cell magnetic order in $\mathrm{YBa}_{2} \mathrm{Cu}_{3} \mathrm{O}_{6.6}$, Phys. Rev. Lett. 118, 097003 (2017).

42 J. Xia, E. Schemm, G. Deutscher, S. A. Kivelson, D. A. Bonn, W. N. Hardy, R. Liang, W. Siemons, G. Koster, M. M. Fejer and A. Kapitulnik, Polar Kerr-effect measurements of the high-temperature $\mathrm{YBa}_{2} \mathrm{Cu}_{3} \mathrm{O}_{6+x}$ superconductor: Evidence for broken symmetry near the pseudogap temperature, Phys. Rev. Lett. 100, 127002 (2008).

43 R.-H. He, M. Hashimoto, H. Karapetyan, J. D. Koralek, J. P. Hinton, J. P. Testaud, V. Nathan, Y. Yoshida, Hong Yao, K. Tanaka, W. Meevasana, R. G. Moore, D. H. Lu, S.-K. Mo, M. Ishikado, H. Eisaki, Z. Hussain, T. P. Devereaux, S. A. Kivelson, J. Orenstein, A. Kapitulnik, and Z.-X. Shen, From a single-band metal to a hightemperature superconductor via two thermal phase transitions, Science 331, 1579 (2011).

44 A. Kapitulnik, Notes on constraints for the observation of Polar Kerr Effect in complex materials, Physica B 460, 151 (2015).

45 W. Cho and S. A. Kivelson, Necessity of time-reversal symmetry breaking for the Polar Kerr Effect in linear response, Phys. Rev. Lett. 116, 093903 (2016).

46 A. Pal, S.R. Dunsiger, K. Akintola, A.C.Y. Fang, A. Elhosary, M. Ishikado, H. Eisaki, and J. E. Sonier, Quasistatic internal magnetic field detected in the pseudogap phase of $\mathrm{Bi}_{2+x} \mathrm{Sr}_{2-x} \mathrm{CaCu}_{2} \mathrm{O}_{8+\delta}$ by $\mu \mathrm{SR}$, Phys. Rev. B 97, 060502 (2018).

47 A. Shekhter, B. J. Ramshaw, R. Liang, W. N. Hardy, D. A. Bonn, F. F. Balakirev, R. D. McDonald, J. B. Betts, S. C. Riggs and A. Migliori, Bounding the pseudogap with a line of phase transitions in $\mathrm{YBa}_{2} \mathrm{Cu}_{3} \mathrm{O}_{6+\delta}$, Nature 498, 75 (2013).

48 S. Badoux, W. Tabis, F. Laliberte, G. Grissonnanche, B. Vignolle, D. Vignolles, J. Beard, D. A. Bonn, W. N. Hardy, R. Liang, N. Doiron-Leyraud, L. Taillefer, and C. Proust, Change of carrier density at the pseudogap critical point of a cuprate superconductor, Nature 531, 210 (2016).

49 B. J. Ramshaw, S. E. Sebastian, R. D. McDonald, J. Day, B. S. Tan, Z. Zhu, J. B. Betts, R. Liang, D. A. Bonn, W. N. Hardy, N. Harrison, Quasiparticle mass enhancement approaching optimal doping in a high-Tc superconductor, Science 348, 317 (2015).

50 M. Randeria, N. Trivedi, A. Moreo, R.T. Scalettar, Pairing and spin gap in the normal state of short coherence length superconductors, Phys. Rev. Lett. 69, 2001 (1992).

51 A.S. Alexandrov, N.F. Mott, Bipolarons, Rep. Prog. Phys. 57, 1197 (1994).

${ }^{52}$ V. J. Emery and S. A. Kivelson, Importance of phase fluctuations in superconductors with small superfluid density,
Nature 374, 434 (1995).

${ }^{53}$ L. Li, Y. Wang, S. Komiya, S. Ono, Y. Ando, G. D. Gu, and N. P. Ong, Diamagnetism and Cooper pairing above Tc in cuprates, Phys. Rev. B 81, 054510 (2010).

${ }^{54}$ H. Alloul, F. Rullier-Albenque, B. Vignolle, D. Colson and A. Forget, Superconducting fluctuations, pseudogap and phase diagram in cuprates, Euro. Phys. Lett. 91, 37005 (2010).

55 P.W. Anderson, The resonating valence bond state in $\mathrm{La}_{2} \mathrm{CuO}_{4}$ and superconductivity, Science, 235, 1196 (1987).

56 C.M. Varma, Non-Fermi-liquid states and pairing instability of a general model of copper oxide metals, Phys. Rev. B 55, 14554 (1997).

57 C.M. Varma, Pseudogap Phase and the quantum-critical point in copper-oxide metals, Phys. Rev. Lett. 83, 3538 (1999).

58 C.M. Varma, Theory of the pseudogap state of the cuprates, Phys. Rev. B 73, 155113 (2006).

59 C. Weber, A. Lauchli, F. Mila, and T. Giamarchi, Orbital currents in extended Hubbard models of high-Tc cuprate superconductors, Phys. Rev. Lett. 102, 017005 (2009).

${ }^{60}$ C. Weber, T. Giamarchi, and C. M. Varma, Phase diagram of a three-orbital model for high-Tc cuprate superconductors, Phys. Rev. Lett. 112, 117001 (2014).

61 R. Thomale and M. Greiter, Numerical analysis of threeband models for $\mathrm{CuO}$ planes as candidates for a spontaneous T-violating orbital current phase, Phys. Rev. B 77, 094511 (2008).

62 S. Nishimoto, E. Jeckelmann, and D. J. Scalapino, Current-current correlations in the three-band model for two-leg CuO ladders: Density-matrix renormalization group study, Phys. Rev. B 79, 205115 (2009).

${ }^{63}$ Y. F. Kung, C.-C. Chen, B. Moritz, S. Johnston, R. Thomale, and T. P. Devereaux, Numerical exploration of spontaneous broken symmetries in multiorbital Hubbard models, Phys. Rev. B 90, 224507 (2014).

64 T.P. Croft, E. Blackburn, J. Kulda, R. Liang, D.A. Bonn, W.N. Hardy, and S.M. Hayden, No evidence for orbital loop currents in charge-ordered $\mathrm{YBa}_{2} \mathrm{Cu}_{3} \mathrm{O}_{6+x}$ from polarized neutron diffraction, Phys. Rev. B 96, 214504 (2017).

65 K.B. Efetov, H. Meier, and C. Pepin, Pseudogap state from quantum criticality, Nat. Phys. 9, 442 (2013).

66 Y. Wang, A.V. Chubukov, Charge-density-wave order with momentum $(2 Q, 0)$ and $(0,2 Q)$ within the spinfermion model: Continuous and discrete symmetry breaking, preemptive composite order, and relation to pseudogap in hole-doped cuprates, Phys. Rev. B 90, 035149 (2014).

67 S. Caprara, C. Di Castro, G. Seibold, and M. Grilli, Dynamical charge density waves rule the phase diagram of cuprates, Phys. Rev. B 95, 224511 (2017).

68 C. Pepin, V.S. de Carvalho, T. Kloss, and X. Montiel, Pseudogap, charge order, and pairing density wave at the hot spots in cuprate superconductors, Phys. Rev. B 90, 195207 (2014)

69 T. Kloss, X. Montiel, V. S. de Carvalho, H. Freire, and C. Pepin, Charge orders, magnetism and pairings in the cuprate superconductors, Rep. Prog. Phys. 79, 084507 (2016).

70 S. Chakravarty, R.B. Laughlin, D.K. Morr, and C. Nayak, Hidden order in the cuprates, Phys. Rev. B 63, 094503 (2001). 
71 I. Affleck and J. B. Marston, Large-n limit of the Heisenberg-Hubbard model: Implications for high-Tc superconductors, Phys. Rev. B 37, 3774 (1988).

72 J. B. Marston and I. Affleck, Large-n limit of the HubbardHeisenberg model, Phys. Rev. B 39, 11538 (1989).

73 J.G. Storey, Simultaneous drop in mean free path and carrier density at the pseudogap onset in high-Tc cuprates, Superconductor Science and Technology 30, 104008 (2017).

74 G. Sharma, S. Tewari, P. Goswami, V. M. Yakovenko, S. Chakravarty, Calculation for polar Kerr effect in hightemperature cuprate superconductors, Phys. Rev. B 93, 075156 (2016).

75 W.A. Atkinson, A.P. Kampf, S. Bulut, Emergence of charge order in a staggered loop-current phase of cuprate high-temperature superconductors, Phys. Rev. B 93, 134517 (2016).

76 I. Makhfudz, d-Density wave scenario description of the new nidden charge order in cuprates, J. Phys. Soc. Jpn. 85, 064701 (2016).

77 H.A. Mook, P. Dai, S.M. Hayden, A. Hiess, J.W. Lynn, S.H. Lee, and F. Dogan, Magnetic order in $\mathrm{YBa}_{2} \mathrm{Cu}_{3} \mathrm{O}_{6+x}$ superconductors, Phys. Rev. B 66, 144513 (2002).

78 H. A. Mook, P. Dai, S.M. Hayden, A. Hiess, S-H. Lee, and F. Dogan, Polarized neutron measurement of magnetic order in $\mathrm{YBa}_{2} \mathrm{Cu}_{3} \mathrm{O}_{6.45}$, Phys. Rev. B 69, 134509 (2004).

79 C. Stock, W. J. L. Buyers, Z. Tun, R. Liang, D. Peets, D. Bonn, W. N. Hardy, and L. Taillefer, Neutron scattering search for static magnetism in oxygen-ordered $\mathrm{YBa}_{2} \mathrm{Cu}_{3} \mathrm{O}_{6.5}$, Phys. Rev. B 66, 024505 (2002).

80 J. E. Sonier, V. Pacradouni, S. A. Sabok-Sayr, W. N. Hardy, D. A. Bonn, R. Liang, and H. A. Mook, Detection of the unusual magnetic orders in the pseudogap region of a high-temperature superconducting $\mathrm{YBa}_{2} \mathrm{Cu}_{3} \mathrm{O}_{6.6}$ crystal by muon-spin relaxation, Phys. Rev. Lett. 103, 167002 (2009).

81 S. Tewari, H.-Y. Kee, C. Nayak, and S. Chakravarty, Spin and current correlation functions in the d-density-wave state of the cuprates. Phys. Rev. B 64, 224516 (2001).

82 M.R. Trunin, Yu.A. Nefyodov, and A.F. Shevchun, Superfluid density in the underdoped $\mathrm{YBa}_{2} \mathrm{Cu}_{3} \mathrm{O}_{7-x}$ : evidence for d-density-wave order of the pseudogap, Phys. Rev. Lett. 92, 067006 (2004).

83 Ar. Abanov, A.V. Chubukov, and J. Schmalian, Quantum-critical theory of the spin-fermion model and its application to cuprates: Normal state analysis, Adv. Phys. 52, 119 (2003).

${ }^{84}$ M.A. Metlitski, and S. Sachdev, Quantum phase transitions in two spatial dimensions. II. Spin density wave order, Phys. Rev. B 82, 075128 (2010).

85 M. Hashimoto, R.-H. He, K. Tanaka, J.-P. Testaud, W. Meevasana, R. G. Moore, D. Lu, H. Yao, Y. Yoshida, H. Eisaki, T. P. Devereaux, Z. Hussain, and Z.-X. Shen, Particle-hole symmetry breaking in the pseudogap state of Bi2201, Nat. Phys. 6, 414 (2010).

86 A. Kaminski, S. Rosenkranz, H.M. Fretwell, M.R. Norman, M. Randeria, J.C. Campuzano, J-M. Park, Z. Z. $\mathrm{Li}$, and H. Raffy, Change of Fermi-surface topology in $\mathrm{Bi}_{2} \mathrm{Sr}_{2} \mathrm{CaCu}_{2} \mathrm{O}_{8+\delta}$ with doping, Phys. Rev. B 73, 174511 (2006).

87 H. Anzai, A. Ino, M. Arita, H. Namatame, M. Taniguchi, M. Ishikado, K. Fujita, S. Ishida, and S. Uchida, Relation between the nodal and antinodal gap and critical tem- perature in superconducting Bi2212, Nat. Com. 4, 1815 (2013).

88 J. Bardeen, L.N. Cooper, and J.R. Schrieffer, Theory of superconductivity, Phys. Rev. 108, 1175 (1957).

89 S.I. Mukhin, Instanton sector of correlated electron systems as the origin of populated pseudo-gap and flat "band" behavior: Analytic solution, J. Supercond. Nov. Mag., 22, 75 (2009)

90 S.I. Mukhin, Spontaneously broken Matsubara's time invariance in fermionic system: Macroscopic quantum ordered state of matter, J. Supercond, Nov. Mag., 24, 1165 (2011)

91 S.I. Mukhin, Negative energy antiferromagnetic instantons forming Cooper-pairing 'glue' and 'hidden order' in high-Tc cuprates, Condensed Matter, 3, 39; 2018.

92 S.A. Brazovskii, S.A. Gordyunin. and N.N. Kirova, An exact solution of the Peierls model with an arbitrary number of electrons in the unit cell, JETP Lett. 31, 487 (1980).

93 J. Mertsching, and H.J. Fischbeck, The Incommensurate Peierls phase of the quasi-one-dimensional Frohlich model with a nearly half-filled band, Phys. Stat. Sol. 103, 783 (1981).

94 K. Machida and M. Fujita, Soliton lattice structure of incommensurate spin-density waves: Application to $\mathrm{Cr}$ and Cr-rich Cr-Mn and Cr-V alloys, Phys. Rev. B 30, 5284 (1984).

95 A.A. Abrikosov, L.P. Gorkov, I.E. Dzyaloshinskii, Methods of quantum field theory in statistical physics, Prentice Hall, New York (1963).

96 M. Abramowitz, and A. Stegun. Handbook of mathematical functions. Dover, New York (1970).

97 A.F. Volkov and Sh.M. Kogan, Collisionless relaxation of the energy gap in superconductors, JETP, 38, 1018 (1974).

98 R.A. Barankov, L.S. Levitov, and B.Z. Spivak, Collective Rabi oscillations and solitons in a time-dependent BCS pairing problem, Phys. Rev. Lett. 93, 160401 (2004).

99 R.A. Barankov and L.S. Levitov, Synchronization in the $B C S$ pairing dynamics as a critical phenomenon, Phys. Rev. Lett. 96, 230403 (2006).

100 E.A. Yuzbashyan, B.L. Altshuler, V.B. Kuznetsov, and V.Z. Enolskii, Nonequilibrium cooper pairing in the nonadiabatic regime, Phys. Rev. B 72, 220503(R) (2005).

101 E.A. Yuzbashyan, B.L. Altshuler, V.B. Kuzentsov, and V. Z. Enolskii, Solution for the dynamics of the BCS and central spin problems. J. Phys. A: Math. Gen. 38, 7831, (2005).

102 E.A. Yuzbashyan and M. Dzero, Dynamical vanishing of the order parameter in a fermionic condensate, Phys. Rev. Lett. 96, 230404 (2006).

103 A. Moor, A.F. Volkov, and K.B. Efetov, Amplitude Higgs mode and admittance in superconductors with a moving condensate, Phys. Rev. Lett. 118, 047001 (2017).

104 V. Galitski, Nonperturbative quantum dynamics of the order parameter in the BCS pairing model. Phys. Rev. B 82, 054511 (2010).

105 R. Matsunaga, Y. I. Hamada, K. Makise, Y. Uzawa, H. Terai, Z. Wang, and R. Shimano, Higgs amplitude mode in the BCS superconductors $N b_{1-x} T i_{x} N$ induced by terahertz pulse excitation, Phys. Rev. Lett. 111, 057002 (2013).

106 R. Matsunaga, N. Tsuji, H. Fujita, A. Sugioka, K. Makise, Y. Uzawa, H. Terai, Z. Wang, H. Aoki, R. Shimano, Light-induced collective pseudospin precession resonating with Higgs mode in a superconductor, Science 345, 1145 (2014). 
107 R.J. Glauber, Coherent and incoherent states of the radiation field, Phys. Rev. 131, 2766 (1963).

108 N.N. Bogoliubov, A new method in the theory of superconductivity I, Soviet Phys. JETP 34, 41 (1958).

109 R. Loudon, The quantum theory of light, Oxford University Press, New York (2000).

110 S.I. Mukhin, and T.R. Galimzyanov, Phys. Rev. B, 100, 081103 (R), 2019.

111 P.G. De Gennes, Superconductivity of metals and alloys, Addison-Wesley, New York (1989).

112 G. Floquet, Sur les équations différentielles linéaires à coefficients périodiques, Ann. de l'Ecole Norm. Sup. 12,
$47(1883)$

113 E.H. Lieb, and D.W. Robinson, The finite group velocity of quantum spin systems, Commun. Math. Physics, 28, 251 (1972).

114 D.W. Robinson, Statistical mechanics of quantum spin systems, Commun. Math. Physics, 7, 337 (1968).

115 P.W. Anderson, Random-phase approximation in the theory of superconductivity, Phys. Rev. 112, 1900 (1957)

116 N.S. Headings, S.M. Hayden, J. Kulda, N.H. Babu, \& D.A. Cardwell, Spin anisotropy of the magnetic excitation in the normal and superconducting states of optimally doped $\mathrm{YBa}_{2} \mathrm{Cu}_{3} \mathrm{O}_{6.9}$ studied by polarized neutron spectroscopy, Phys. Rev. B 84, 104513 (2011). 